\title{
EQUIPMENT FOR ROUTINE SPECTRAL TRANSMISSION AND REFLECTION MEASUREMENTS
}

\author{
By H. J. McNicholas
}

\begin{abstract}
This paper presents a description of an assembly of apparatus which has been in use at the Bureau of Standards since August, 1924, for routine spectral transmission and reflection measurements. The Koenig-Martens spectrophotometer is used with different light sources, each source being designed especially to meet the required conditions of illumination on the sample, in the measurement of the following quantities as functions of wave length:

1. The unidirectional normal transmission of an optically homogeneous (nondiffusing) material in the form of a plate with plane-parallel surfaces.

2. The normal brightness of a sample by reflected light, relative to the same for a standard material, when both sample and standard are under equal and completely diffused illuminations.

The sources for transmission measurements are three separate white-lined inclosures, each just large enough to contain, respectively, two 400-watt gasfilled incandescent lamps, two helium lamps, and a quartz mercury-vapor lamp. These sources are conveniently and quickly interchangeable, so that any one of them may be used separately for specific purposes.

A uniform distribution of 156 small gas-filled incandescent lamps on a white diffusely reflecting hemispherical surface provides a source of illumination for reflection measurements which is shown to be equivalent to the desired ideal completely diffused condition.

Accessory equipment includes ventilation apparatus, convenient devices for handling the transmission and reflection samples, standard sectored disks for use with the spectrophotometer, and apparatus for temperature control of liquid transmission samples.

The theory of the Koenig-Martens spectrophotometer is given, along with a discussion of some possible sources of error in the spectrophotometric measurements.

Tests of the accuracy in the measurement of the specified quantities are described.

\section{CONTENTS}

I. Introduction

II. Description of light sources__ 798

1. Source for reflection measurements_______. 798

(a) Construction_.._. 798

(b) Ventilation system

(c) Cooling of sample....... 801

(d) Illumination and observation of the sample _...... 802

2. Sources for transmission measurements___________ 803

(a) Principle of construction_._. 803

(b) Tungsten lamp source_______ 805

(c) Helium lamp source

(d) Mercury-vapor lamp source

(e) Use of transmission sources...


III. Accessory apparatus

1. Mechanism for the insertion and interchange of the sample and comparison standard in reflection measurements.....

2. Devices for the support and shifting of transmission samples_

3. Thermostat for temperature control of liquids..........

4. Sectored-disk attachment

IV. Koenig-Martins spectrophotometer

1. Theory of the instrument.

2. Use of the instrument for polarization measurements........

3. Use of the instrument for reflection and transmission measurements.

(a) The polarization problem: Simplified formulas_.....

(b) Propagation of error through the formulas _........

(c) Choice of formula for routine work

(d) Use of one quadrant of the circular scale on nicol mounting

(e) Procedure in measurements

4. Further remarks on the instrument and its adjustments

(a) Stray light

(b) Photometric field

(c) Wave-length scale

(d) Circular scale

(e) General remarks

V. Use of equipment and tests of its performance

1. Transmission measurements.

2. Reflection measurements

(a) Reflection standards_.......... 850

(b) Adequacy of absolute illumination on sample_..... 852

(c) Equivalence of the illumination to the ideal completely diffused condition.

\section{INTRODUCTION}

The measurement of the transmissive and reflective properties of various materials for wave lengths throughout the visible spectral range is a matter of fundamental importance in the work of a colorimetry laboratory, for the data thus obtained under given conditions of illumination and observation constitute a description of the material which is unique and sufficient for the purposes of colorimetry. When multiplied into the spectral energy distribution of the source of illumination, the resulting data are a representation of the entire physical stimulus of the sensation of color (neglecting simultaneous and successive contrast effects) and form the fundamental basis for color specification. The use of these data with the psychophysical data of color vision in the computation of various colorimetric functions has been considered in detail by others. In the establishment of material color standards for various purposes, the spectrophotometric data form a suitable and adequate record of the colors, so that the reproduction of the standards at some future time may be verified without depending on the permanence of the materials originally used. The utility of spectrophotometric equipment and methods in 
many fields of research is being recognized by an increasing number of investigators.

A serious disadvantage inherent in the use of visual methods of spectrophotometry lies in the decreased precision of measurement attainable toward both ends of the visible spectrum as a result of the decreased sensibility of the eye to these spectral regions. The difficulty is further enhanced in the blue and violet regions by the relatively lower energy emission of the sources of light commonly used. In some applications of the spectrophotometric data, as in the transformation into the trilinear coordinates of colorimetry, it is often of importance to obtain accurate transmission or reflection data over the spectral range from 380 or 400 to $740 \mathrm{~m} \mu$. Sometimes the range from 420 to $720 \mathrm{~m} \mu$ is sufficient. Unless recourse is had to radiometric methods, the above-mentioned difficulty in the visual method can only be overcome by providing sources of light of sufficient intensity so that the desired photometric precision and range of measurement are obtained.

The transmissive and reflective properties of various materials depend not only on the nature and structure of the material itself, but may also vary considerably with the conditions of illumination and observation. Various transmission and reflection coefficients corresponding to these conditions may therefore be defined and measured to serve particular purposes.

In transmission measurements we are most frequently concerned with the unidirectional normal transmission of light through an optically homogeneous (nondiffusing) medium in the form of a plate with plane-parallel polished surfaces. The beam of light is in this case incident in a direction perpendicular to the surface of the sample; it is partly reflected at the surfaces in a regular manner and partly transmitted by the medium without scattering or appreciable deviation from its original path. The quantity to be measured is the ratio of the total transmitted to the total incident light and is thus defined as the transmission (considered now as a magnitude).

In the case of reflection measurements, on the other hand, we are usually concerned with diffusing media, such as papers, painted surfaces, dyed fabrics, etc., for which the incident light is generally reflected in all directions from the surface of the sample. The conditions of illumination and observation must be definitely specified, if unambiguous and reproducible results are to be obtained.

The specification of the illumination for reflection measurements is based upon the following considerations:

1. The absolute illumination on the sample must be sufficient for precise spectrophotometric measurements over the desired range of wave lengths, but the sample must be protected at the same time from excessive heating by the source. 
2. The angular distribution of the incident light must be definitely determined by the construction of the source, so that the conditions of illumination may be readily reproduced.

3. It is desirable that the conditions of illumination adopted be also representative of some natural condition of illumination, under which, for instance, the color of various materials is ordinarily observed.

It is difficult to realize all the desired conditions of illumination simultaneously by the use of a single commercial type of lamp. Such illumination will generally be insufficient for spectrophotometric purposes. It is necessary, therefore, to resort to multidirectional illumination of some sort, whereby the combined effect of a number of different light sources is obtained. If no restrictions be placed on the solid angular extent of the incident luminous flux, it is advantageous to place the light sources in an inclosure surrounding the sample, the walls of which are made of a good diffusing material of high reflectance. ${ }^{1}$ Furthermore, to make the condition of illumination definite and reproducible, it is expedient to arrange the light sources with reference to the sample so that the illumination on the sample is for all practical purposes completely diffused.

No attempt will be made in this paper to discuss the advantages or disadvantages of different conditions of illumination for colorimetric or other purposes. Completely diffused illumination as compared with unidirectional illumination is, perhaps, a closer approximation to the average condition under which the color of a sample is observed (by reflected light) in a well-lighted room or in the open, when the sample is shaded from the direct rays of the sun. In regard to the spectrophotometric measurements, a source for the diffused illumination of the sample may readily be constructed to comply with all of the desired specifications.

If it be desired to measure the total reflected light, some method for the integration of the light flux in all directions from the sample is required. Although suitable methods for this purpose may be applied in certain cases, it is usually most convenient to measure the light reflected in a specified direction from the surface of the sample; and, indeed, this corresponds to the actual condition of use of the eye in observing the color of materials. In this case, however, only a small portion of the total reflected light is directly received by the eye, or by the spectrophotometric observing system, so that a greater light flux on the sample is required in order to obtain the same photometric field brightnesses (hence, the same precision and range of measurement) as may readily be obtained in the above-defined case of unidirectional transmission, wherein the beam of incident light is not broken up or scattered by the sample.

1 Reflectance is defined as the ratio of the total reflected to the total incident light, without regard to directions of incidence or reflection. 
The method of observation herein adopted is to view the sample in a direction approximately normal to its surface. The symmetry of both the illumination and the direction of observation with respect to the surface of the sample is an advantage in spectral reflection measurements, which will be evident later in the discussion of these measurements.

The equipment to be described is designed, then, for the measurement of the following quantities:

1. The unidirectional normal transmission of an optically homogenous material.

2. The normal brightness of a sample by reflected light relative to the same for a standard material, when both sample and standard are under equal and completely diffused illuminations.

In the design and construction of the apparatus for these measurements considerable attention has been given to the matter of convenience in the arrangement and location of the various parts, in the handling of the samples and recording of observations, and to the stability and permanence of the adjustments, in order that the work could be carried on at any time as a routine daily procedure, if desired, with the greatest possible facility and speed and without preliminary adjustments or frequent attention to the working parts. The assurance that a given amount of work may be accomplished in a specified time, without annoying interruptions or uncertainties arising from failures or irregularities in the performance of the apparatus, is a source of the greatest satisfaction to those having to do with a considerable amount of this work.

The equipment has now been in service for nearly four years. ${ }^{2}$ Such provisions as have been made to facilitate and expedite the measurements are, if seemingly elaborate, fully warranted, nevertheless, by the use which has been made of this equipment. In addition to determinations of the spectral transmissive or reflective properties of more than 200 samples of various materials submitted during the past four years by outside industrial establishments, private individuals, and other Government departments, an even greater demand for the spectrophotometric data has arisen in various research projects carried on at the Bureau of Standards, particularly those originating in the colorimetry laboratory. These investigations often call for the highest precision and accuracy attainable in the spectrophotometric measurements.

\footnotetext{
2 The equipment herein described replaces the old illumination apparatus for transmission and reflection measurements which was in use in the colorimetry section of the bureau prior to the summer of 1924, and described in part in the following papers: B. S. Tech. Paper No. 148, p. 5; 1920; B. S. Tech. Paper No. 167, p. 20; 1920; B. S. Sci. Paper No. 440, pp. 126, 143; 1922; J. O. S. A. and R. S. I., 8, p. 203; 1924.
} 


\section{DESCRIPTION OF LIGHT SOURCES}

The salient features and general arrangement of the spectrophotometer and illumination apparatus are best illustrated by the photographs in Figures 1 and 2 and by the diagram in Figure 3 . The apparatus is built around a wooden framework placed in the center of a small room, which may be darkened, is well ventilated, and devoted exclusively to spectrophotometric work. All switches, rheostats, and other instruments for the control of the lamps, motors, etc., are conveniently assembled on a wall board. Detailed information on the construction of the light sources and accessory parts is given in Figures 4, 5, and 6, to which frequent reference will be made in the description. In the various figures different parts are referred to by number, and a given part is numbered the same in whichever figure and view it may be shown. A general key to the construction is provided, which serves for all of the figures and supplements the description to be found in the text. (See legend to fig. 4.)

The specific requirements in regard to the illumination of the sample, in the particular type of reflection and transmission measurements to be made, are most advantageously met by the use of separate sources especially designed for each kind of work. The advantages of this plan will be brought out in the course of the following description.

\section{SOURCE FOR REFLECTION MEASUREMENTS}

The source for reflection work is an attempt to realize the ideal condition of completely diffused illumination on the sample to a sufficient approximation for practical purposes, combined with an illumination which is sufficient for precise spectrophotometric measurements throughout the desired spectral range.

(a) Construction.- These purposes are served in a satisfactory manner by the following construction:

The sample and comparison standard are placed side by side near the center, and forming a part, of the base of a hollow steel hemisphere (40 $\mathrm{cm}$ in diameter), the inner (concave) surface of which is coated with white porcelain enamel (baked), over which a film of magnesium oxide is deposited. ${ }^{3}$ The white diffusely reflecting surface thus obtained is studded with 156 small gas-filled incandescent lamps (described in key to construction of apparatus), which are uniformly

\footnotetext{
3 The white magnesium oxide "smoke" is easily deposited on a porcelain (or other) surface by holding the surface about 2 inches above the flame of the burning metal. Magnesium metal shavings (as used for Grignard reaction) are generally used, although the ribbon form of the metal may be more convenient in some cases. The shavings are burned in a suitable vessel of brick or iron. The white porcelain surface is first applied to the steel surface in order to avoid the necessity of thick and fully opaque deposits of the oxide, which are more difficult to put on and liable to crack. The relatively good refiecting surface of porcelain is more easily "covered" by the oxide. The eyes of the operator should be protected from the oxide flame by use of dark-yellow glasses.
} 


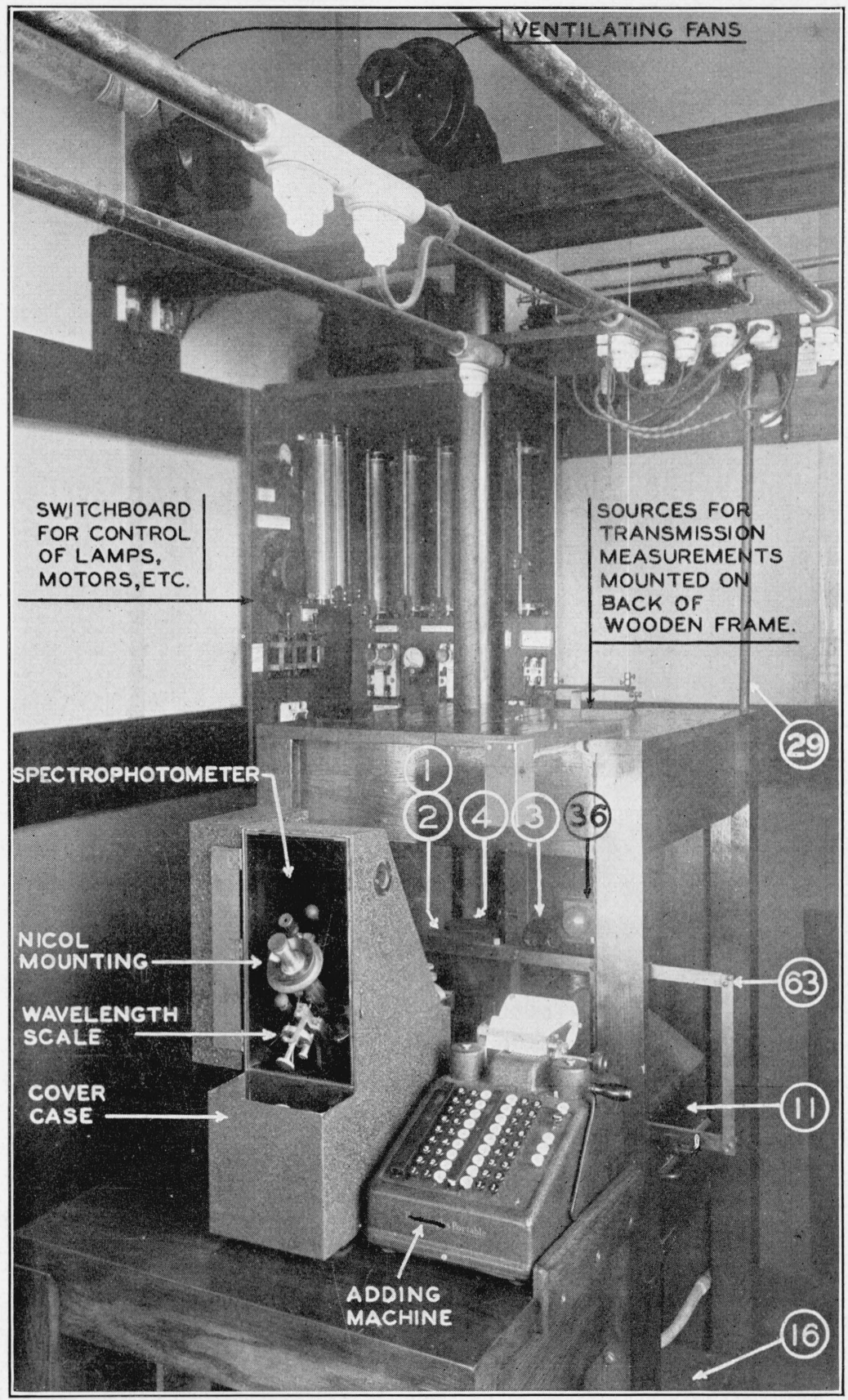

FIG. 1.-General view of equipment for visual spectrophotometry 


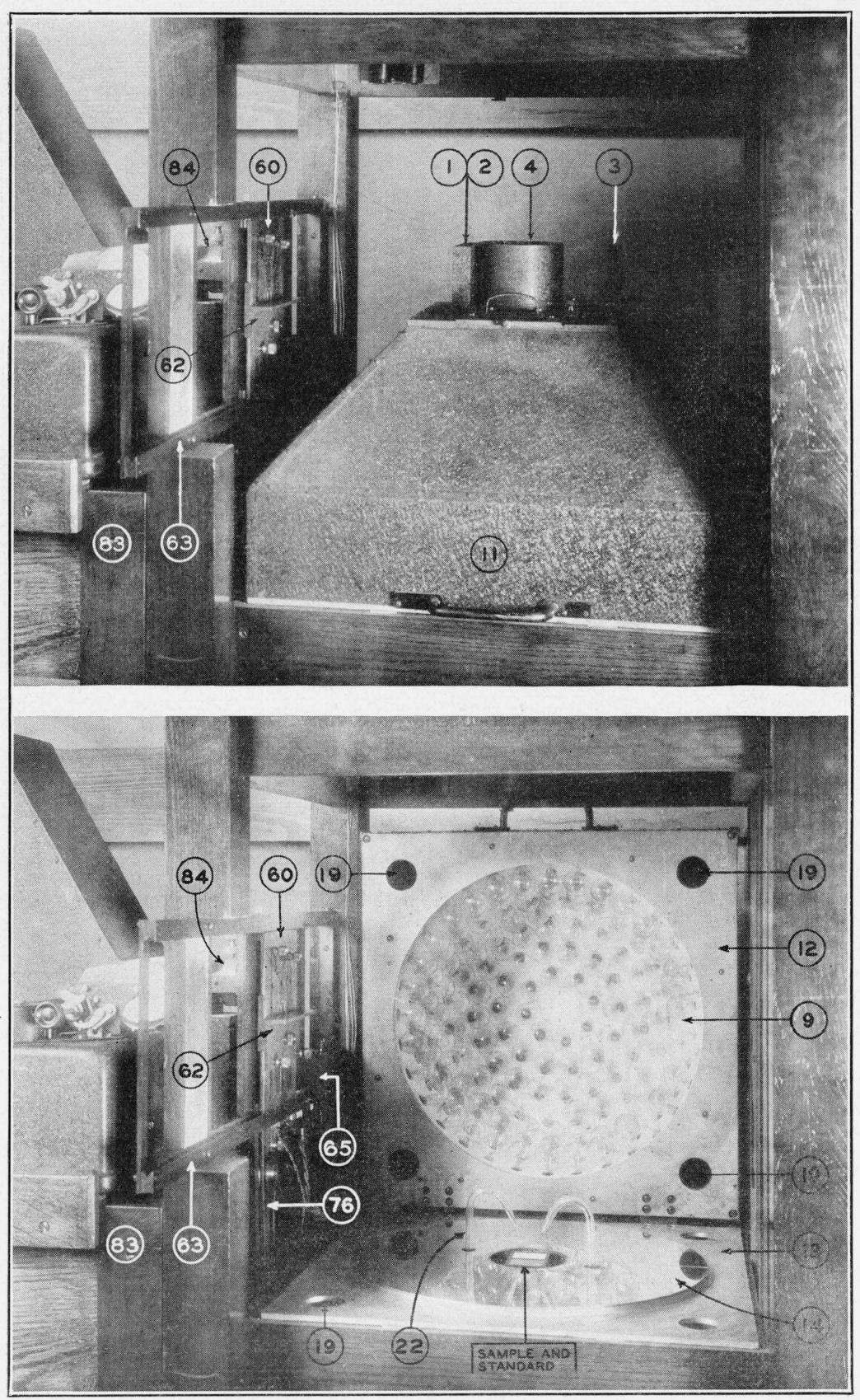

FIG. 2.-Views of hemispherical source for reflection measurements Hemisphere raised in lower photosraph, showing arran rement of lamps, mirror base, and parts of ventilation system 


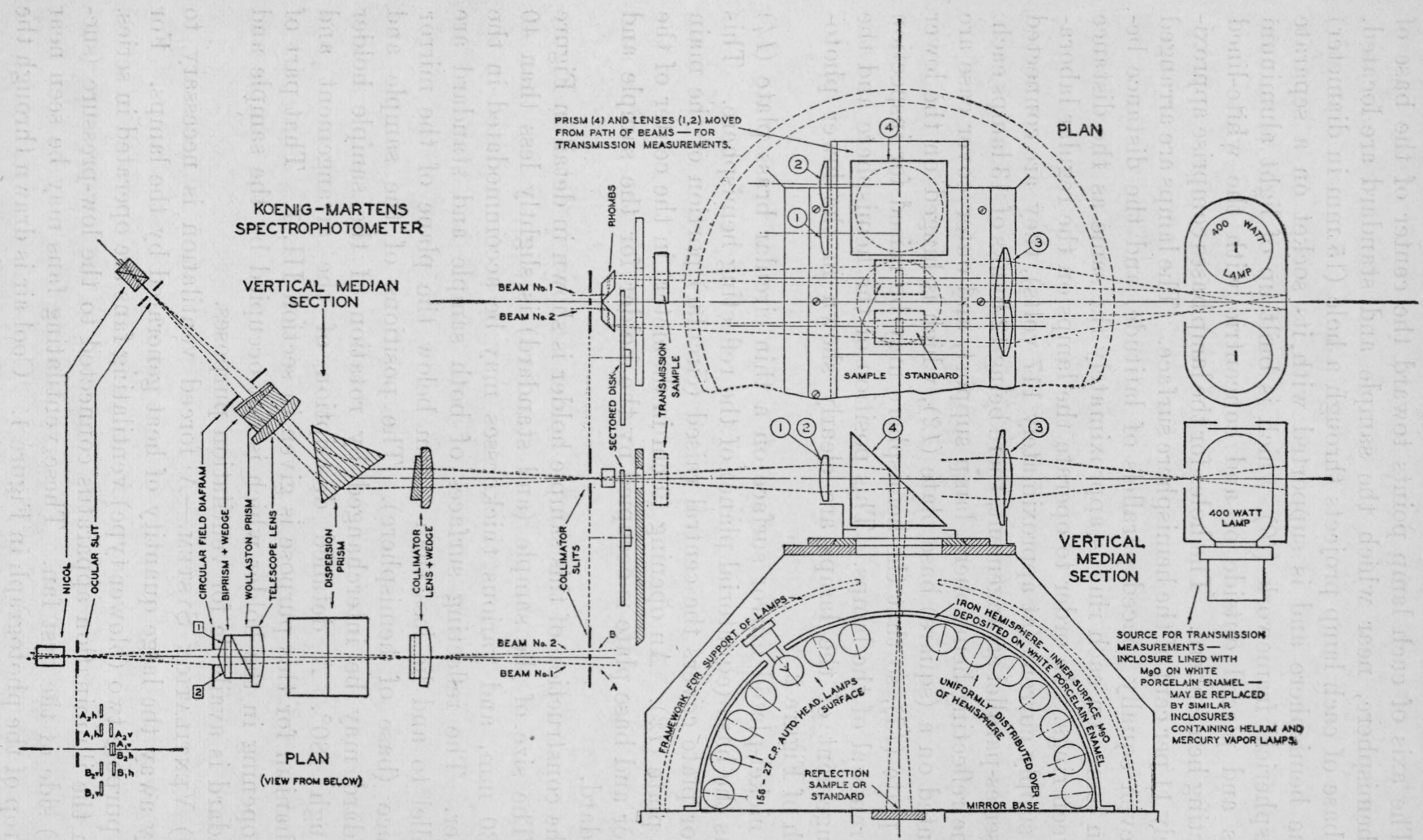

Fig. 3.-General arrangement of spectrophotometer and separate light sources for transmission and reflection measurements (Uniform scale not used throughout the diagram) 
distributed over the surface and mounted as shown in Figures 3 and 4. The axis of each lamp points toward the center of the base of the hemisphere, near which the sample and standard are located. The base of each lamp projects through a hole (15 $\mathrm{mm}$ in diameter) in the hemisphere and is supported with its socket on a separate hemispherical framework (10), which is built up of light aluminum strips and placed outside of and concentric with the white-lined reflecting hemisphere. The holes for the lamp bases comprise approximately 11 per cent of the hemisphere surface. The lamps are arranged on seven equally spaced parallels of latitude, and the distance between lamps on each ring is approximately the same as the distance between rings. In order to operate the lamps on the regular laboratory supply current (at approximately 117 volts), they are connected in a series-parallel arrangement, there being 12 banks of 13 lamps each.

The reflecting hemisphere, lamp support, and outer cover case are mounted on a (square) base plate (12), which is hinged on the lower base plate (13), so that the hemisphere may be raised for inspection and renewal of the lamps. This position of the hemisphere and the arrangement of the lamps are clearly shown in the lower photograph of Figure 2.

A nickel-plated mirror surface on a thin circular brass plate (14) forms the base (equatorial plane) of the reflecting hemisphere. This mirror plate covers the central raised (circular) portion of the main base plate (12). An opening (9 $\mathrm{cm}$ in diameter) in the center of the mirror and base plate is occupied by the holder for the sample and standard.

The construction of this sample holder is shown in detail in Figure 5. The size of the sample (and standard) is slightly less than 40 by $30 \mathrm{~mm}$, and various thicknesses may be accommodated in the holder. The reflecting surfaces of both sample and standard are parallel to and lie less than $1 \mathrm{~mm}$ below the plane of the mirror surface (base of hemisphere). The positions of the sample and standard may be interchanged by rotation of the sample holder through $180^{\circ}$. A detailed description of the arrangement and mechanism for this purpose is given in section III, 1. That part of the opening in the holder which is not occupied by the sample and standard is available for ventilation purposes.

(b) Ventilation System.-A forced ventilation is necessary to carry away the large quantity of heat generated by the lamps. For this purpose two (blower type) ventilating fans are operated in series, with the illumination apparatus connected to the low-pressure (suction) side of the first fan. These ventilating fans may be seen near the top of the photograph in Figure 1. Cool air is drawn through the apparatus and the heated air discharged from the room. 
It has been found necessary to filter all the air used for ventilation purposes in order to remove dust and dirt, which would otherwise be gradually deposited on the white magnesium oxide coating of the hemisphere and soon appreciably decrease its reflectance. This filtering is accomplished in a satisfactory manner by passing the air through a few overlapping layers of absorbent cotton. The construction of the air filter (16) is shown in Figure 4. The layers of cotton are held in place between two square frames of wood covered with a coarse-mesh wire screen.

The course of the air stream through the apparatus may be followed by reference to the various views in Figure 4. Air from the room, after passing through the filter, flows through the pipe (17) into the compartment (18). This air, which is still cool, now flows around the sample and standard into the hemispherical inclosure and out through the many small annular openings around the bases of the lamps into an outer compartment inclosed by the cover case (11). In the lower photograph of Figure 2, four holes (each $4 \mathrm{~cm}$ in diameter) may be seen in the base (12) of this cover case, one located near each corner. In the same photograph, and in view $B$ of Figure 4 , four holes of equal diameter and similarly located are shown in the base plate (13). When the hemisphere is closed (as in the upper photograph of fig. 2 , or in view $A$ of fig. 4 ), the holes in both base plates coincide, permitting the flow of air into four pipes (20), which lead from the four openings in base (13) to the larger pipe (21), and thence direct to the ventilating fans.

(c) Cooling of Sample.-The inflow of air about the sample and standard aids considerably in the cooling of the sample and surrounding metal parts, and the outflow around the bases of the lamps is most effective in carrying away the heat generated by the lamps.

The intense irradiation of the sample would cause some materials to become too hot, however, if an additional cooling were not provided. This additional cooling is supplied by a high-velocity jet of air (from the laboratory compressed-air supply line) which is directed from above on the sample through one of the nozzles shown in view $A$ of Figure 4 and in the lower photograph of Figure 2. These nozzles are made of clear fused-quartz tubes, which absorb very little of the infra-red heat rays, and hence do not preheat the air stream passing through. Likewise, being transparent to the visible rays, they have no appreciable effect on the angular distribution of the light incident on the sample and standard.

Here, again, the entering air may be dirty or oily and, if not cleaned before using, would soon deposit an appreciable amount of dirt on the reflection sample. The quartz tubes are inserted into brass tubes below the base of the hemisphere; these tubes then lead through a valve (23) (purpose described below) to the air filter, and thence to the 
supply line. The construction of this air filter is essentially the same as that of the filter described above; it is not shown, however, in any of the figures.

With the lamps operating at normal voltage, there is an expenditure of about 2,800 watts of electric power. The ventilation system (with the lamps in operation) draws 13 cubic feet of air per minute through the hemisphere. With the room temperature at $30^{\circ} \mathrm{C}$, a thermometer held in the outgoing stream of air registered $66^{\circ} \mathrm{C}$. Two mercury-in-glass thermometers, one with a clear bulb and the other with a blackened bulb, when supported in a vertical position within the hemispherical inclosure with the bottom of each bulb in contact with a reflection sample of black paper, registered $52^{\circ}$ and $56^{\circ} \mathrm{C}$., respectively, after temperature conditions had become steady with both air streams in operation. When the thermometer bulbs were raised to the center of the hemispherical inclosure, the corresponding readings were $70^{\circ}$ and $90^{\circ} \mathrm{C}$., respectively.

The actual temperature attained by a sample under these conditions has not been directly measured (as with a thermocouple embedded in the body of the material). The temperature is not excessive, however, and the sample usually feels just warm to the touch. The reflective properties of certain dyed materials occasionally change slightly under the intense irradiation from the hemisphere lamps. This change is probably an actinic effect, however, and not a burning or scorching of the material.

(d) Illumination and Observation of the Sample.-With the hemisphere lamps all operating at normal voltage, the absolute brightness of a magnesium carbonate sample observed normal to its surface was found to be 10.8 lamberts. ${ }^{4}$ The brightness of this material under completely diffused illumination is practically the same for all angles of view, and its reflectance is close to 98 per cent; hence, the illumination on the sample is 11 lumens per square centimeter. ${ }^{5}$

The path of the beams of light from the sample and standard to the spectrophotometer is clearly shown in the two views of Figure 3. The collimator (entrant) slits of the spectrophotometer are focused on larger slits near the pole of the hemisphere by the lenses (1) and (2). These hemisphere slits may be seen in the lower photograph of

\footnotetext{
4 The absolute brightness was measured in the following manner:

From the construction of the hemispherical illumination apparatus, as depicted in Figure 4, A, it may be seen that it is possible to remove a circular area $5 \mathrm{~cm}$ in diameter centering at the pole of the reflecting hemisphere and containing the pair of slits (15). This being done, then, a Martens photometer was arranged so that, with the aid of a small total-reflection prism and a lens, the brightness of the magnesium-carbonate sample could be compared in the photometric field with a standard brightness set up outside of the hemisphere. Proper corrections were applied for reflection losses at the optical surfaces between the carbonate and the photometer. A neutral absorbing screen of known transmission was used to decrease the difference between the measured brightness and the standard. The same method was used for the absolute-brightness measurements mentioned later in connection with the description of the transmission sources.

${ }^{3}$ It is of interest to note that the normal illumination of a surface from the direct rays of the noon summer sun (at Washington) is approximately 10 lumens per square centimeter.
} 
Figure 2. (See also at (15), fig. 4.) The area of the sample which is observed through the spectrophotometer is semicircular in form and equal approximately to $2 \mathrm{~cm}^{2}$. A convergent beam of light normally reflected from this area of the sample passes through the corresponding hemisphere and collimator slits and illuminates one half of the spectrophotometric field. Light reflected from the comparison standard, similarly, illuminates the other half of the field. The comparison of the brightnesses of the sample and standard is fully discussed in sections IV and V.

\section{SOURCES FOR TRANSMISSION MEASUREMENTS}

(a) Principle of Construction.-An intense beam of light is desired for transmission measurements, incident on the sample in a direction approximately normal to its surface. The method often used in spectrophotometry is to focus the incandescent filament of a lamp, or the crater of an arc, through the sample onto the collimator slit of the spectrophotometer. This method may prove very troublesome, however, if, as a result of small irregularities or a nonparallelism of the surfaces of the sample, the image of the source on the collimator slit is shifted when the sample is inserted or removed. Errors of serious magnitude may result in this case if the source is not of uniform intrinsic brightness over a sufficiently large area. Moreover, when a parallel beam through the sample is not used a correction to the illumination of the collimator slit may be necessary, involving the thickness and index of refraction of the sample.

The ideal source for accurate and reliable transmission measurements is a uniformly bright, diffusely emitting surface of sufficient extension so that a beam of light from this source, passing through the collimator slit and filling the photometric field of the spectrophotometer, may then be wholly, or in part, shifted by the slight irregularities of the sample, without changing the illumination of the photometric field. With this source the thickness of the sample has in itself no effect on the illumination of the field.

In the measurements to be made it is assumed that there is no considerable scattering or large-angle refraction of the light during its passage through the sample; that is, we are dealing ideally with the transmission of clear, optically homogeneous materials. Different methods are required for diffusing media. No materials are optically perfect, however, and many of the samples to be measured will slightly scatter or refract the transmitted light. There is, therefore, a considerable advantage in the use of the extended source, for then, if a narrow pencil of rays originating at a given point of the source and forming part of the beam entering the spectrophotometer is scattered out of the beam after the insertion of the sample, it should be 
fully replaced by an equal pencil of rays scattered into the beam from an adjacent part of the source. ${ }^{6}$

This type of source offers no particular difficulties in construction, and the required brightness for spectrophotometric work may be readily obtained. Making use of the well-known properties of an inclosure, the walls of which reflect light in approximate accordance with the cosine law, the average total illumination of the wall is given by

$$
\frac{F}{A(1-R)}
$$

where $A$ is the area of the wall, $R$ its average reflectance, and $F$ the total light flux projected into or generated within the inclosure. By placing lamps in as small an inclosure as possible, then, with walls of the highest reflectance attainable, the maximum illumination and brightness of the interior surface is obtained. A portion of this surface, viewed through a suitable aperture into the inclosure, serves as a practical realization of the desired ideal type of source. Care must be taken, however, to insure a sufficiently uniform brightness distribution over an area somewhat larger than the observed portion of the interior wall. Referring to the above formula, it is seen that the average direct illumination of the wall is $F / A$, but that, as a result of multiple reflections within the inclosure, the total illumination is at least ten times greater, if an average reflectance of 0.9 for the interior surface of the inclosure be assumed. Thus, approximately 90 per cent of the total illumination of the observed spot and surrounding area is due to the multiply reflected indirect component which, in consequence of the reflective properties of the wall, is nearly completely diffused and sufficiently uniform over the required area. The lamps should be arranged inside the inclosure so that the remaining 10 per cent of the total illumination of the observed region, which is received directly from the lamps, is as nearly uniform as possible. Obviously a high value of $R$ is desirable, not only to obtain the highest possible brightness but also to increase the proportion of the indirect illumination, thus insuring a more uniform brightness distribution over the observed portion of the interior surface. The aperture into

\footnotetext{
6 This statement is but an application of the Helmholtz reciprocity law to this case of transmission meas. urements. The application of the law in the analogous case of diffuse reflection has been fully discussed in B. S. J. of Research, 1, p. 29 (No. 3); 1928.

To make a rigorous application of the law in this case, however, an assumption must first be made in regard to the diffusive properties of the sample. Because of the impossibility of interchanging the directions of the incident and scattered light in strict compliance with the reciprocity law, we must assume that the quantity and angular distribution of the diffused transmitted light for unidirectional normal illumination are practically the same when the light is incident on either surface of the sample. This is generally a valid assumption for the type of sample with which we are concerned. Then, to compensate completely for the light scattered out of the beam in the case here considered, it is only necessary that the solid angle subtended at the sample by the effective aperture into the lamp inclosure be equal to or larger than the solid angular extent of the slightly diffused transmitted light for strictly unidirectional normal illumination. Under these conditions the light entering the spectrophometer is exactly equal to that which would enter the instrument if the sample were made nondiffusing without changing its transmission.
} 
the inclosure, through which light passes to the spectrophotomoter, should be as small as possible in order to obtain the highest possible average reflectance of the interior wall. On the other hand, the effective aperture into the inclosure should be large enough to take care of the slight diffusive properties or irregularities of the sample.

The hemispherical source for reflection measurements (previously described) would obviously also serve for transmission measurements, in which case the sample and comparison standard could be replaced by (say) two blocks of magnesium carbonate. As we are primarily interested, however, in a diffusely emitting surface of high and uniform brightness over a limited area, and not particularly concerned with the manner in which this surface is illuminated, a great advantage over the above hemispherical construction is gained by use of a small inclosure, with fewer lamps and a lesser expenditure of electric power.

The sources to be described are designed on the above principles. They are not intended, however, to be of sufficient extension to compensate completely for the light scattered by imperfect samples having badly scratched or poorly polished surfaces. Appreciably lower transmissions may be obtained for such samples than would be obtained with a fully extended (hemispherical) source.

The construction of the sources and their arrangement is shown in Figure 4 (section view $\mathrm{A}$ and elevation view $\mathrm{B}$ ). Three different lamp inclosures are there shown, containing gas-filled tungsten-filament incandescent lamps, helium lamps, and a quartz mercuryvapor lamp, respectively. They are permanently mounted on a plate (30), which slides between two vertical guide bars (31) and is held in any position by the counterweight (32). A handle (33) and a spring catch (34) are provided, so that either lamp inclosure may be conveniently and easily raised or lowered into position for use in transmission measurements.

(b) Tungsten Lamp Source.-The middle inclosure (also illustrated in fig. 3) is an iron box (36) coated on its inner surface with white porcelain enamel and "smoked" with magnesium oxide. The box is self-ventilating and just large enough to inclose the bulbs of two 400-watt gas-filled tungsten-filament incandescent lamps (35), the bases of the lamps being outside the white-lined inclosure. A sufficient separation between the lamps is allowed for the passage of two beams of light from the back wall of the box through an aperture in the front wall and thence to the spectrophotometer.

This source, giving a continuous spectrum, is used for the greater part of the transmission work. With the lamps operating at normal voltage the brightness of the inner surface, as viewed through the aperture, is 88 lamberts. The lamps are operated in series on 220 volts direct current. The 400 -watt lamps may be replaced by 1,000 - 
watt lamps of approximately the same type and size, if desired. A forced ventilation would then be necessary, however, to prevent an excessive temperature in the lamps, thus greatly shortening their life. An inclosure of the type described in this section could be designed to give a greater interior brightness than is obtained with the present construction.

(c) Helium Lamp Source.-The form of the two helium lamps (37) is clearly shown in views $\mathrm{A}$ and $\mathrm{B}$ of Figure 4 . The discharge tubes are $5 \mathrm{~mm}$ in diameter and contain helium gas at a pressure of approximately $10 \mathrm{~mm}$ (of mercury). They are connected in series, forming a total tube length of $80 \mathrm{~cm}$, and are operated on a highvoltage transformer. ${ }^{7}$ Two aluminum rods (shown in fig. 1) make permanent electrical connection to the lamps by sliding contact with an overhead high-voltage line.

With the exception of their terminal bulbs, the helium lamps are inclosed in a small rectangular box (38), the upper, lower, and front sides of which are made of asbestos board (for electrical-insulation purposes combined with good heat-resisting qualities), and the remaining sides are made of aluminum. Two small holes in the top and bottom sides provide for a sufficient ventilation. The inner wall of this inclosure is covered with a thick deposit of magnesium oxide, and an aperture in the front side is provided for the emergence of the light beams to the spectrophotometer. The brightness of the interior surface is approximately 0.5 lambert.

(d) Mercury-vapor Lamp Source.-The lower source (fig. 4) is a quartz mercury-vapor lamp (40), with the straight portion of its tube inclosed as much as possible in an iron box (41), the inner surface of which is also porcelain enameled and coated with magnesium oxide. Light for the sample and spectrophotometer is taken from the back cylindrical surface of the inclosure through an aperture in the front wall.

The mercury lamp, with its inclosing box, is mounted on a circular plate (42), which is in turn supported on a hollow bearing, so that the whole lamp may be turned into a vertical position for the purpose of starting. The lamp is operated on the 220 -volt directcurrent supply line, and the brightness of the interior curved surface is approximately 51 lamberts when the terminal voltage across the arc reaches its usual value of 120 volts.

(e) Use of Transmission Sources.-The course of the light beams from the transmission source to the spectrophotometer is best shown in the upper view of Figure 3. The transmission sample is in position in one of the beams; and the large total-reflection

7 To operate these lamps in series, a 1-kilowatt, 20,000-volt transformer which was available in the laboratory is used with considerable resistance in the primary circuit. A voltage of 10,000 or less is sufficient for this purpose. 
prism (4), with lenses (1) and (2), is moved out of the path of the beams from the transmission sources. (In the lower view these lenses and the prism are in place for reflection measurements.)

The lens combination (3) serves the following purposes: (1) The two beams to the spectrophotometer are taken from approximately the same area of the wall of the lamp inclosure, so that any variations in the relative brightness distribution over the interior surface of any one of the inclosures affects both beams equally; (2) the collimator slits of the spectrophotometer are focused approximately in the plane of the aperture into the lamp inclosure. The latter circumstance, combined with the overlapping of the beams inside the inclosure, is an advantage in the design of these sources. The smallest possible aperture into the inclosure may thus be used, so that a maximum area of the wall is conserved for reflecting purposes; also, less free space is needed inside the inclosure for the light beams.

The apertures of the lens combination ( $(3)$ and of the lamp inclosures are made large enough to permit small displacements of either beam (corresponding to the introduction of an imperfect sample) without obstructing the free passage of the beam to the spectrophotometer.

\section{ACCESSORY APPARATUS}

\section{MECHANISM FOR THE INSERTION AND INTERCHANGE OF THE SAMPLE AND STANDARD IN REFLECTION MEASUREMENTS}

The adopted method of use of the spectrophotometer (described in Sections IV and V) requires an interchange of the positions of the sample and standard during the eourse of a reflection measurement, and it is desirable that this operation be performed rapidly and in a manner convenient to the observer.

In addition to the diagram in Figure 5, the sample holder is shown in position for reflection measurements in views $\mathrm{A}$ and $\mathrm{C}$ of Figure 4. The interchange of the sample and standard is effected by rotation of the holder through $180^{\circ}$. For this purpose the gear teeth on the outer edge of the holder are in mesh with the gear teeth of a drivewheel (45), which is in turn connected through bevel gears (46) to the drive shaft (47). This shaft may be rotated by the observer from his position at the spectrophotometer by a handwheel (50), which is conveniently located to the left of the spectrophotometer cover case (fig. 1) and connected by pulleys $(48,49)$ and belt to the shaft $(47)$. Suitable stops $(6)$ on the holder prevent its rotation through more than $180^{\circ}$.

The mechanism for the removal (or insertion) of the holder from the illumination apparatus is explained as follows: From the section view $A$ of Figure 4 it is clear that the weight of the sample holder is carried on the circular shaft (51). This shaft has a rack cut into 
one side, so that it may be moved up or down by the pinion (52) attached to the shaft (53). This rack-and-pinion arrangement is operated by the handwheel (54) on the shaft (53). The latter is normally prevented from turning by the clamp (55). To remove the sample and standard, then, this clamp is released and the sample holder lowered until it rests upon a carriage (56). This position of the holder is shown in view $\mathrm{E}$ of Figure 4. The carriage with the

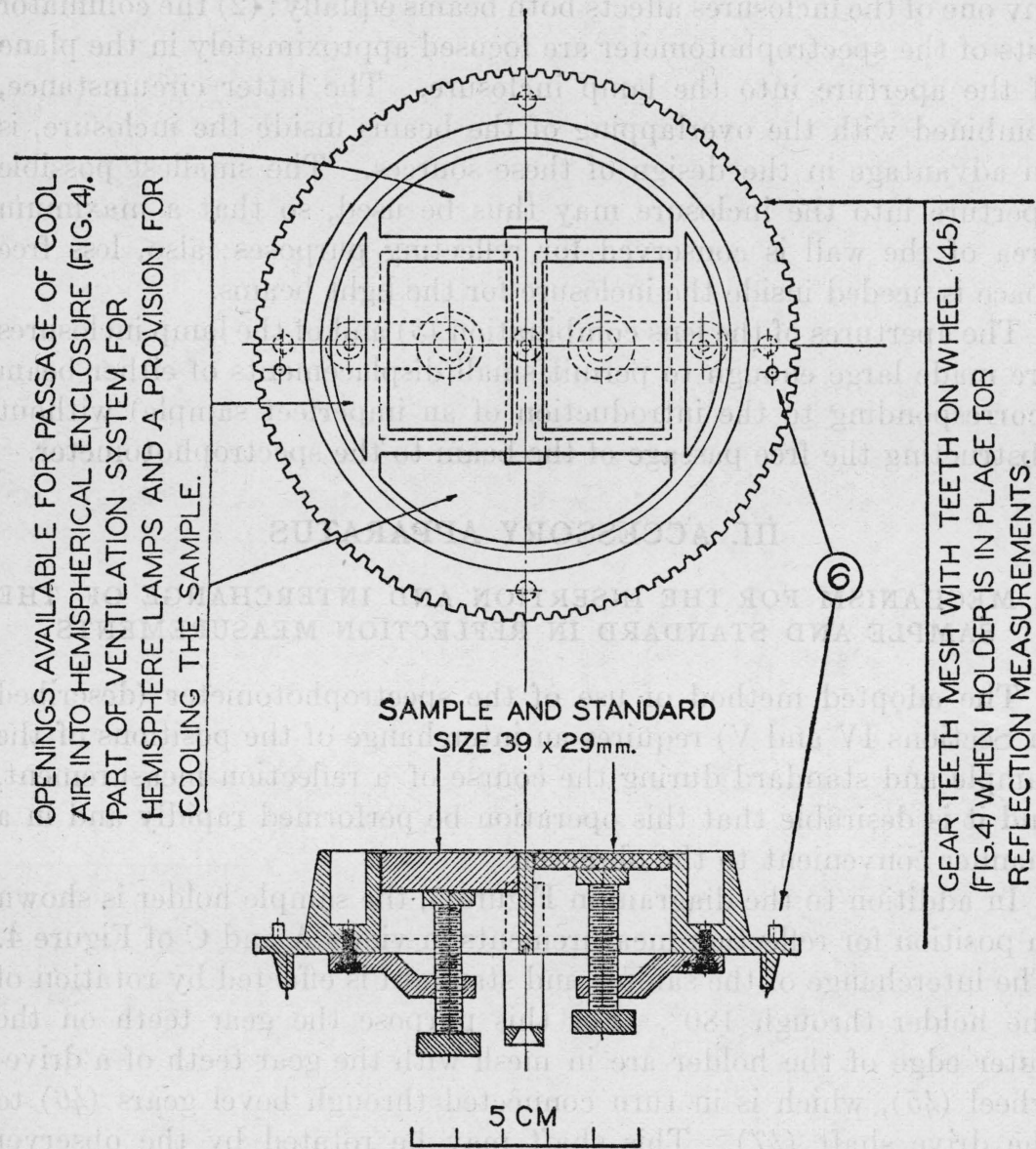

FIG. 5.-Construction of holder for reflection sample and comparison standard

(Part No. 5 of fig. 4 )

holder may now be moved along a track (57) by pulling out the rod (58) until the holder comes under the door (59) (shown open in views $\mathrm{C}$ and $\mathrm{E})$. The holder can then be lifted from the carriage and taken out for inspection or change of sample. In the reverse operation of inserting the sample, the holder is raised from the carriage by the shaft (51), and the gear teeth on the holder again come into mesh with the teeth of the driving gear. 
As the sample and standard are interchanged, the valve (23) is simultaneously operated through the levers (24) and pins (6), so that the high-velocity cooling-air jet is first cut off and then shifted from one of the nozzles (22) to the other. The operation of this valve mechanism may be followed in the views $\mathrm{C}, \mathrm{D}$, and $\mathrm{E}$. It is designed so that the observer need only be concerned with the proper position of the sample holder on its carriage when being inserted into the apparatus; consequently the air stream is always directed onto the sample and never on the standard. Two reasons for this provision. may be mentioned: (1) The comparison standard may always consist of a material requiring no special cooling, so that a greater volume of air is thereby available for the cooling of the sample; (2) the highvelocity air stream impinging continuously on the surface of the standard would be detrimental in the case of some materials which might be used.

\section{DEVICES FOR THE SUPPORT AND SHIFTING OF TRANSMISSION SAMPLES}

The samples submitted for transmission measurements are likely to vary considerably in size, shape, and thickness, so that a somewhat flexible arrangement for the support of the sample in the beam of light is required, which may be readily adapted to accommodate different samples. As in the case of reflection measurements, the method of use of the spectrophotometer requires the shifting of the transmission sample from one beam to the other, and it is equally desirable in this case that the shifting operation be performed rapidly and in a manner convenient to the observer.

Views of the sample support are shown in Figures 4 and 6, including a vertical median section in Figure 4, A. A brass plate (60) carries a double platform $(61,62)$, which may be raised or lowered to accommodate the size of the sample and to center it vertically in the beam of light. The long platform (61) is removable, leaving a shorter platform (62), which serves for the majority of samples and does not interfere with the raising of the hemisphere. Three apertures $(68)$ in the plate $(60)$ allow the passage of the light beams to the spectrophotometer. The plate $(60)$ fits into $\mathrm{V}$-shaped grooves in two horizontal guide bars (63), forming a track along which the holder may be moved. For this purpose a rack (64) and a pinion (in the gear box (65)) are provided and operated by a handwheel (66), conveniently placed to the left of the observer. As the holder is moved between the stops $\left(6^{\prime \prime}\right)$ the sample (placed before the central aperture) is shifted from one beam to the other. Upon removal of one of these stops the whole sample support may be moved to the end of the track (63), a provision which is very convenient for the mounting and adjustment of the sample on the platform. This position of the holder is shown in Figure 6 .

$109431^{\circ}-28-2$ 


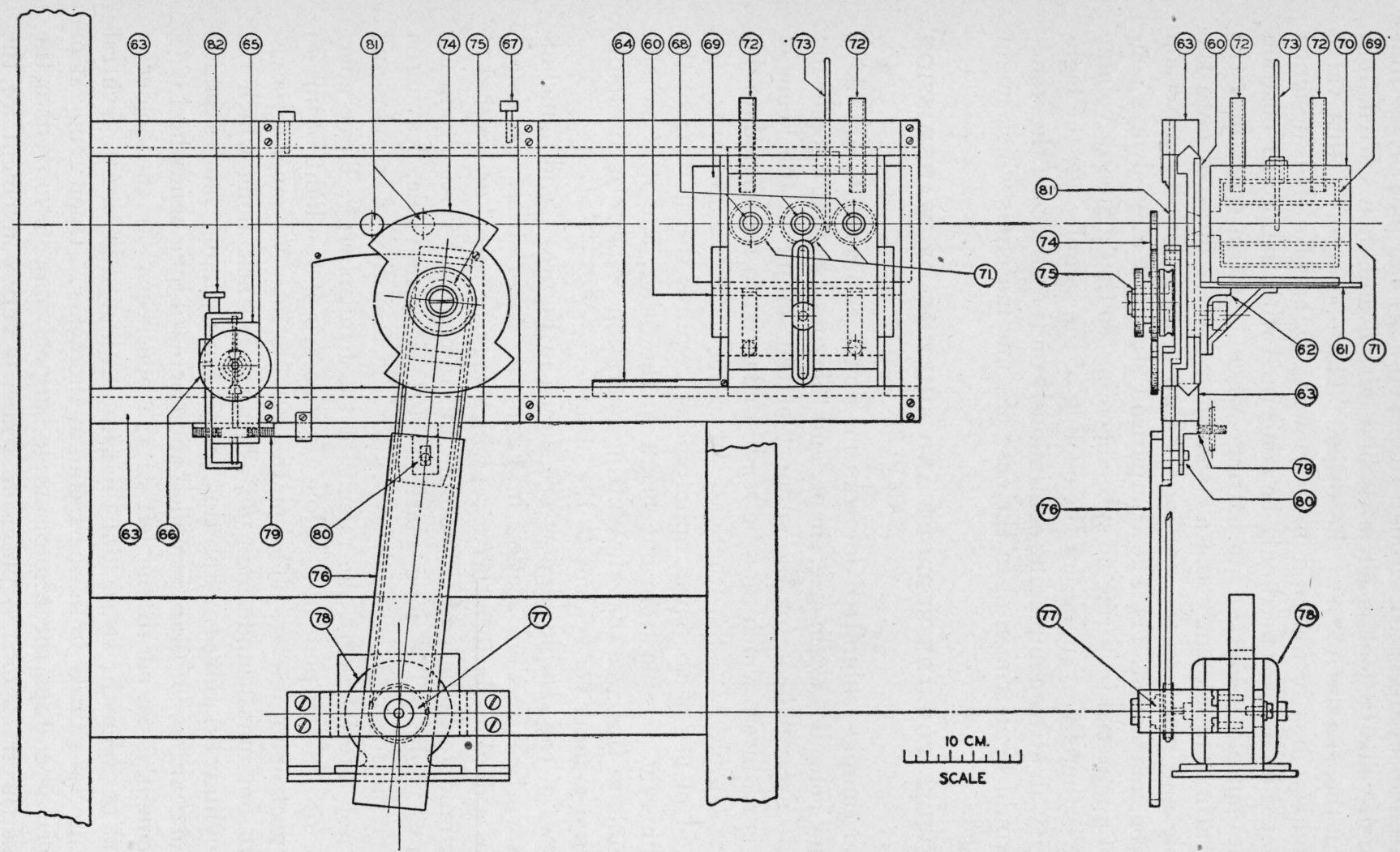

FIG. 6.-Elevation views showing: (1) Sectored disk attachment and shifting mechanism; (2) constant-temperature box for cells containing liquids, mounted in proper position on support for transmission samples 
In the same figure a constant-temperature box (69) is also shown mounted in position on the platform of the sample holder. This box is designed particularly for transmission measurements on liquids, such as inorganic salt and dye solutions, in which it is desired to control the temperature of the solution and its solvent to within a degree or two and to cover a range of temperatures approximately $20^{\circ}$ above and below the room temperature. The rectangular brass box (69) is covered with a heat-insulating material (70) and has three cylindrical brass tubes (71) passing through, as shown. Water from a thermostat-controlled bath (described later) may be circulated

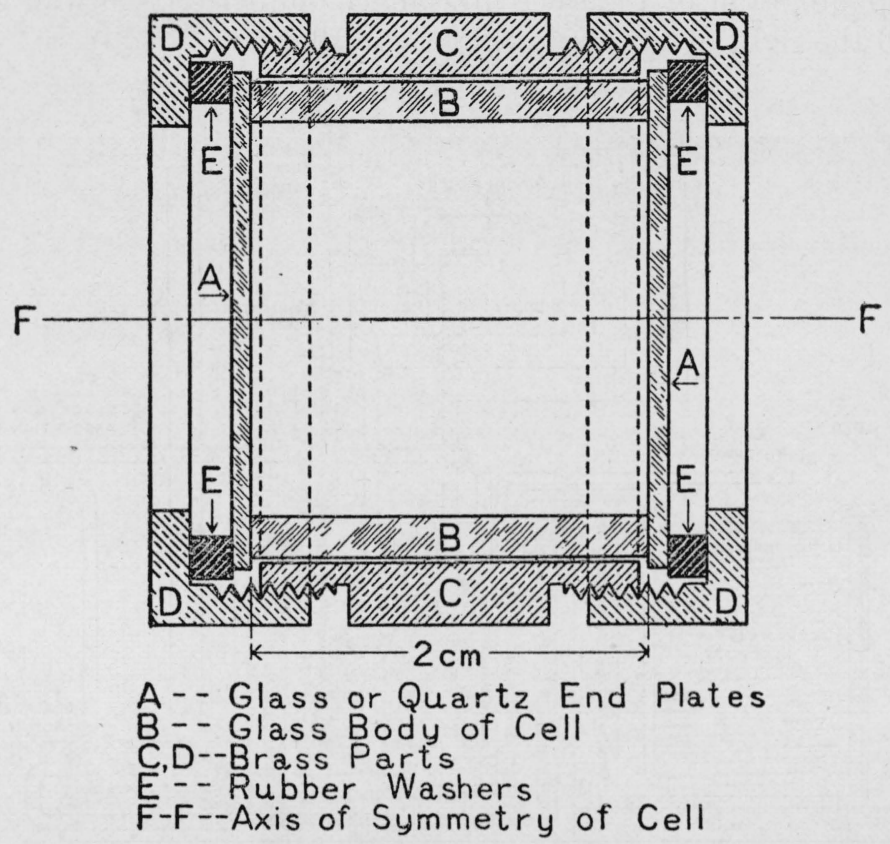

FIG. 7.-Type of cell used in the measurement of the transmissive properties of liquids

(Reproduced from B. S. Sci. Paper No. 440)

through the box, thus maintaining the walls of the tubes at an approximately constant temperature, indicated by the thermometer (73).

A cylindrical cell (construction fully shown in fig. 7) containing the solution to be measured may be inserted in the central tube of the constant-temperature box with similar cells containing the solvent in the outer tubes. The brass sides of the cell are in contact with the brass walls of the box, and the reading of the thermometer is taken as the temperature of the solution and solvent. While this method would not be satisfactory at temperatures differing greatly from room temperature, it is quite suitable for the purposes to which it is applied. 
When the sample holder with the constant-temperature box is moved into position in the light beams, the rack (64) engages with its pinion gear, and, as the solution is moved from one beam to the other, one of the solvent cells is always centered in the opposite beam.

\section{THERMOSTAT FOR TEIMPERATURE CONTROL OF LIQUIDS}

A suitable arrangement for this purpose has previously been described in Bureau of Standards Scientific Paper No. 440. The same arrangement, with slight modifications, is installed with the present equipment and is shown in Figure 8. The apparatus does not appear in the photograph of Figure 1, it being mounted on the wall of the room to the right of the equipment there shown.

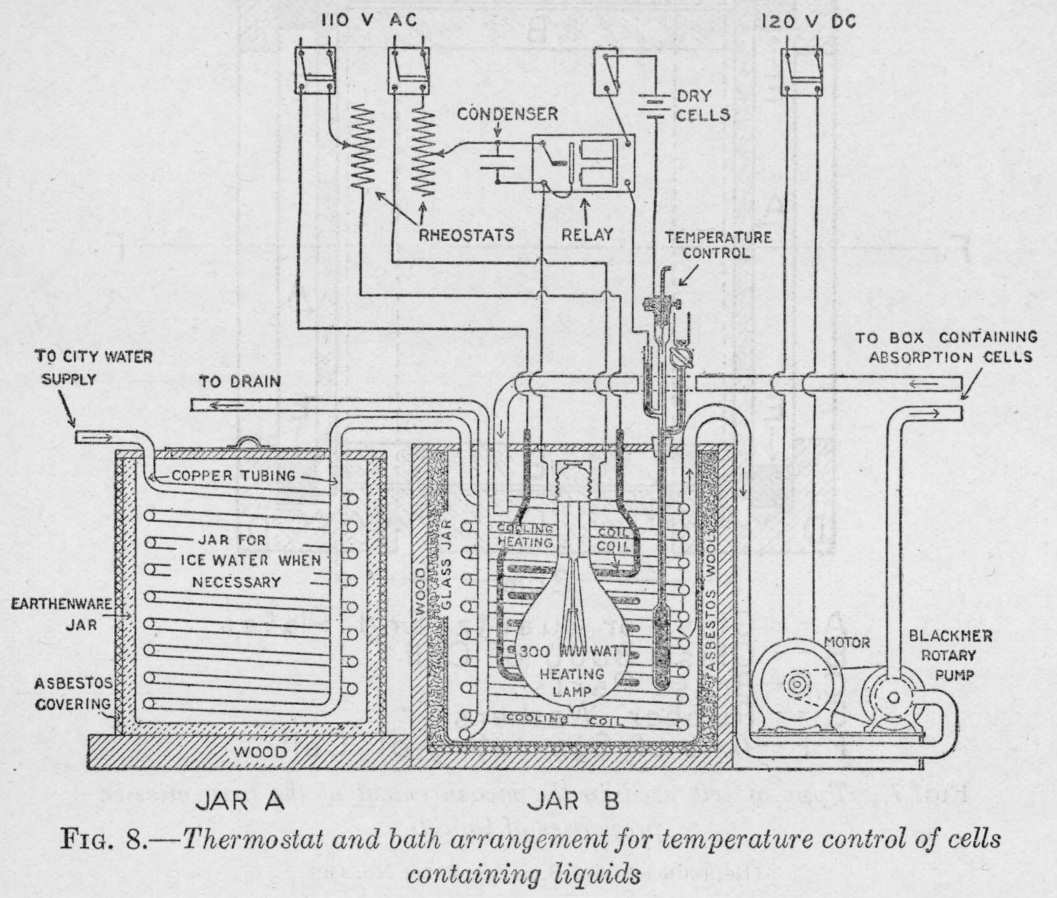

Water from jar $B$ is pumped through a few feet of rubber tubing to the constant-temperature box surrounding the transmission cells, and back again to the same jar. This water is maintained at a constant temperature in the following way: A steady stream of cold water (from the city supply) passes through the copper cooling coils in jars $A$ and $B$ and discharges into the drain. This water may be further cooled, if necessary, by use of ice water in jar $A$, and the volume of water flowing through the coil regulated in accordance with the amount of cooling desired.

Heat is supplied by a 300 -watt gas-filled incandescent lamp suspended in jar $B$. The use of a lamp for this purpose affords a quick 
means for adding heat to the water, for the radiant energy is instantaneously absorbed by the water over a large surface in contact with the lamp bulb. The current through this lamp is switched on and off as required by the thermostat acting through the relay. When the water in jar $B$ is too cool, the contact between the narrow mercury column and a platinum wire in the thermostat is broken by the contraction of the mercury. This interrupts the current through the magnets of the relay, releasing the relay lever and closing the circuit through the lamp. The water in jar $B$ now heats up until the expansion of the mercury again closes the circuit through the relay magnets and opens the circuit through the lamp. The continued action of the thermostat, while the pump keeps the water in circulation through the jar and constant-temperature box, maintains the temperature of the circulating water constant within the required limits. The temperature is regulated by raising or lowering the platinum contact wire in the thermostat until the thermometer in the constant-temperature box reads the desired temperature.

When a temperature well above room temperature is desired, better regulation is obtained by sending a constant current through the heating coil to compensate for a part of the radiation and conduction losses in the system. In this case the cold-water cooling is not required.

\section{SECTORED-DISK ATTACHMENT}

The equipment also includes a set of standard sectored disks of fixed aperture, the transmissions of which are accurately known by mechanical calibration. ${ }^{8}$ These transmission values are approximately $80,70,60,50,10$, and 1 per cent, respectively. By combinations of two disks the range of transmissions can be extended to values between 50 and 10 per cent.

The use of these sectored disks in the spectrophotometric measurements is fully discussed in section V, 1. Briefly, they serve two purposes: (1) To test and maintain a check upon the accuracy of the spectrophotometer, and (2) to extend the range of the instrument accurately below 1 per cent, by use of the 10 or 1 per cent disk in the beam opposite the sample. This procedure reduces the difference between the brightnesses compared in the photometric field, with consequent advantages in the measurement of low transmission or reflection coefficients.

Two views of the sectored-disk attachment are shown in Figure 6, and a section through the axis of rotation of the disk and driving motor is contained in Figure 4, A. These views clearly illustrate the construction and mounting of the disk and the position of the

8 This calibration was made by the Bureau of Standards division of weights and measures and consists in the measurement of the angular opening in each disk. From these data the transmissions of the rapidly rotating disks (assuming the Plateau-Talbot law) are known. 
attachment relative to the other parts of the apparatus. The central hole of each disk fits without play on a circular boss carried by the driving pulley. Two small holes in the disk register with two pins projecting from the base of the pulley, in order to prevent slip and possible loosening of the nut due to inertia of the disk during acceleration or deceleration. Two disks may be mounted together on the axle. A 50 per cent sectored disk is shown in position covering one of the apertures (81) through which the beams of light pass to the spectrophotometer. The other aperture remains clear.

The disk may be shifted from one beam to the other by a rackand-pinion arrangement (79), which is operated through the gear box (65) and handwheel (66). The same handwheel is also used for the shifting of the transmission sample; and, by proper adjustment of the vertical position of the knob (82) the disk attachment may be placed in gear so as to shift simultaneously either with the sample or in the opposite direction. In the latter case the sectored disk is always in the beam opposite the sample, so that the transmission of the sample is measured relative to that of the disk.

\section{KOENIG-MARTENS SPECTROPHOTOMETER}

The type of instrument used for the spectrophotometric measurements is described in papers by Koenig, ${ }^{9}$ Martens, ${ }^{10}$ Martens and Greenbaum, ${ }^{11}$ and has become known as the Koenig-Martens spectrophotometer. The arrangement of some of the optical parts of the instrument ${ }^{12}$ in the possession of this laboratory differs slightly, however, from that described in the paper last cited. The optical system is shown in Figure 3; and, to conserve space in this figure, the plan of the optical system is displaced from its proper position relative to the plan of the illumination apparatus.

The theory and method of use of the instrument is given in the following pages, along with a discussion of some possible sources of error in the spectrophotometric measurements.

\section{THEORY OF THE INSTRUMENT}

A diaphragm placed over the jaws of the (horizontal) collimator slit divides this slit into two parts ( $A$ and $B$, fig. 3 ), which are of equal length and width and separated by one slit length from each other. The double collimator slit thus formed is provided with an accurate bilateral adjustment for width.

A Wollaston (double image) prism and a biprism are permanently mounted as a unit on the telescope lens, with the line of intersection

\footnotetext{
9 Ann. der Phys. and Chem., 53, p. 783; 1894.

10 Verh. der Deut. Phys. Gesellsch., 1, p. 280; 1899.

11 Ann. der Phys. (4), 12, p. 984; 1903.

12 Purchased from Schmidt and Haensch about 1912.
} 


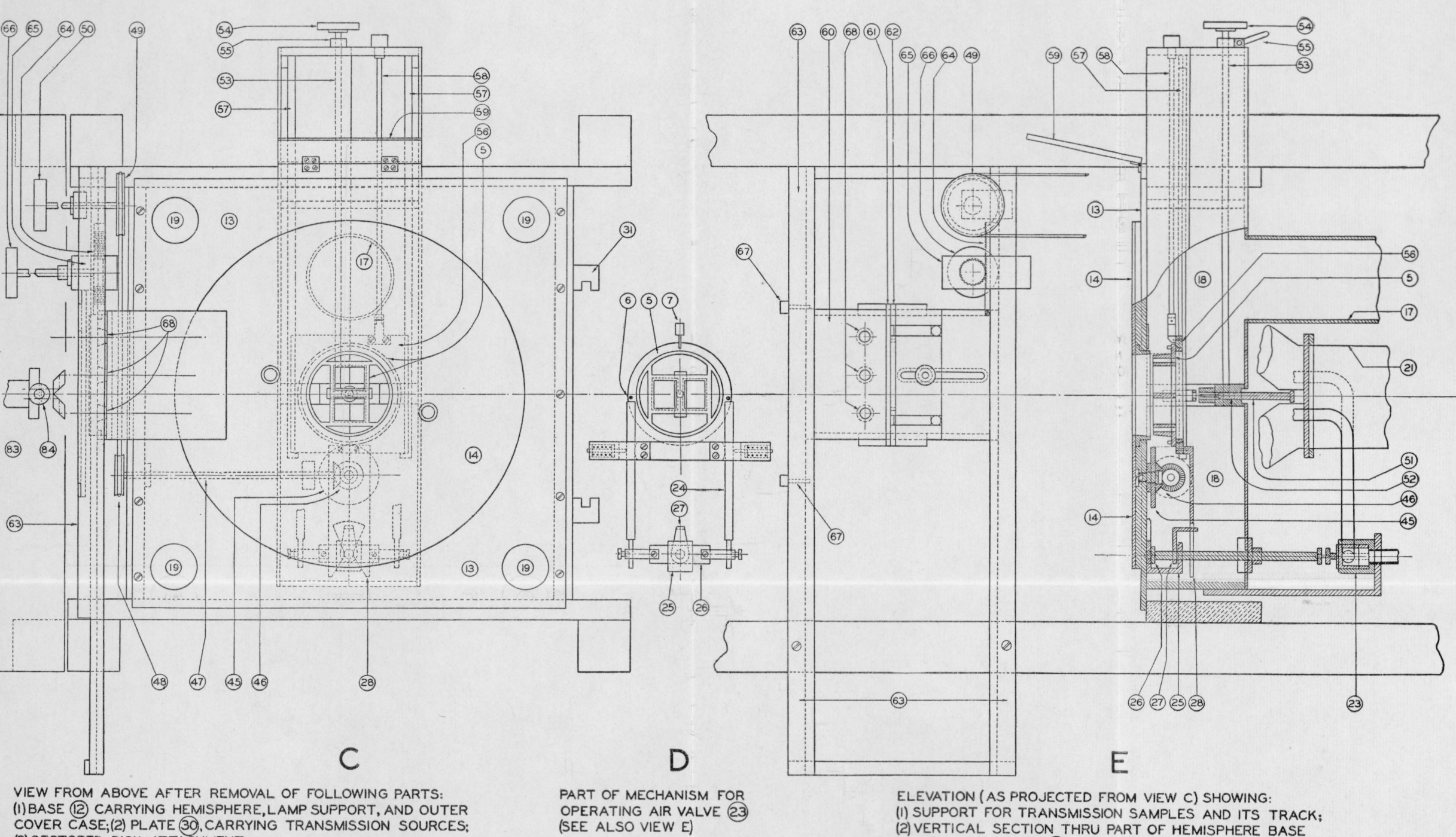

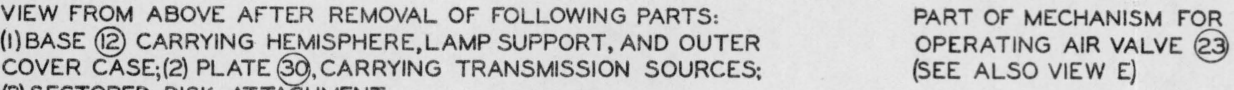
(3) SECTORED DISK ATTACHMENT

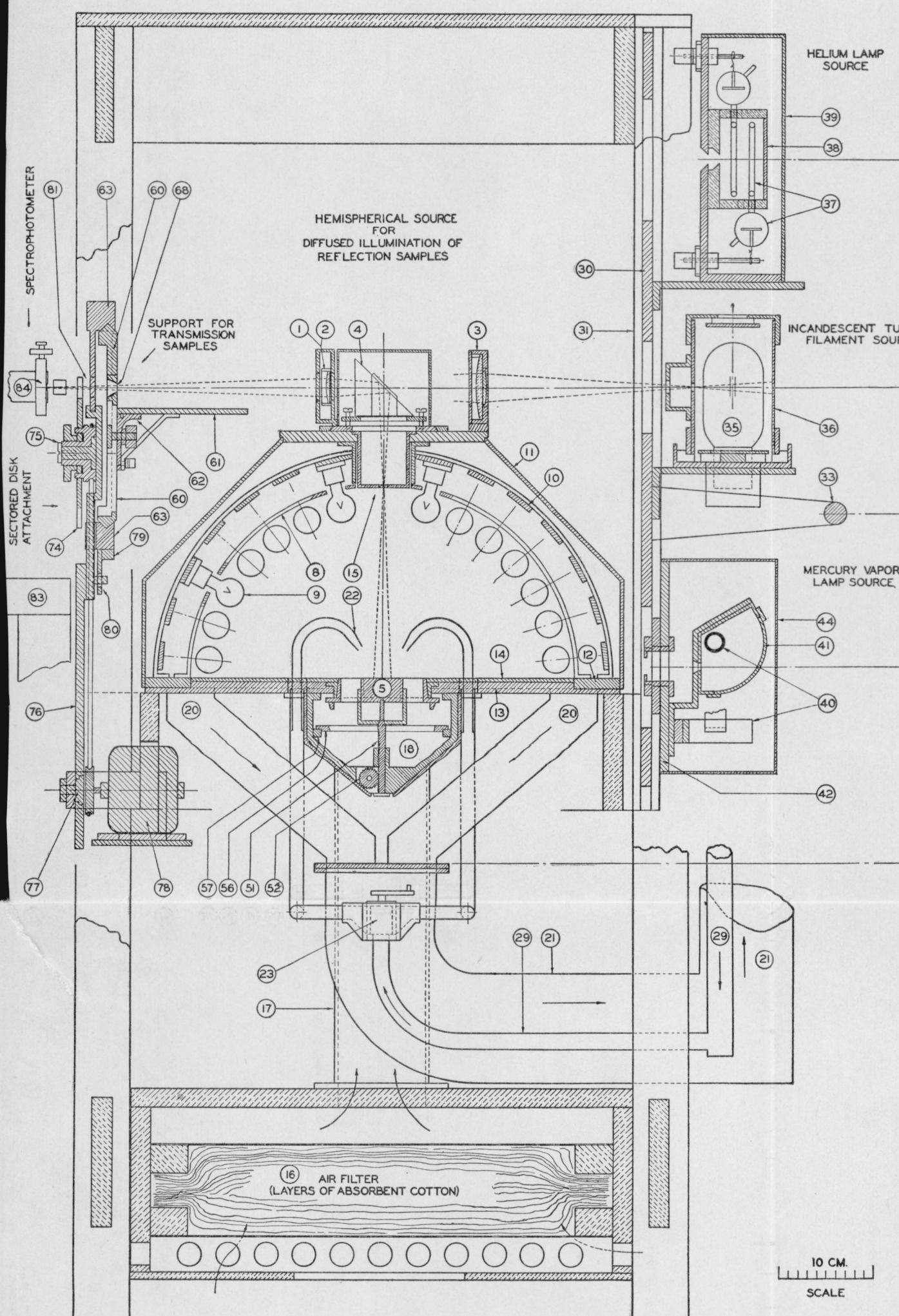

\section{A}

VERTICAL SECTION THRU POLE OF HEMISPHERE AND CENTER OF

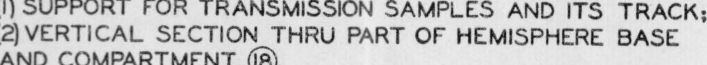

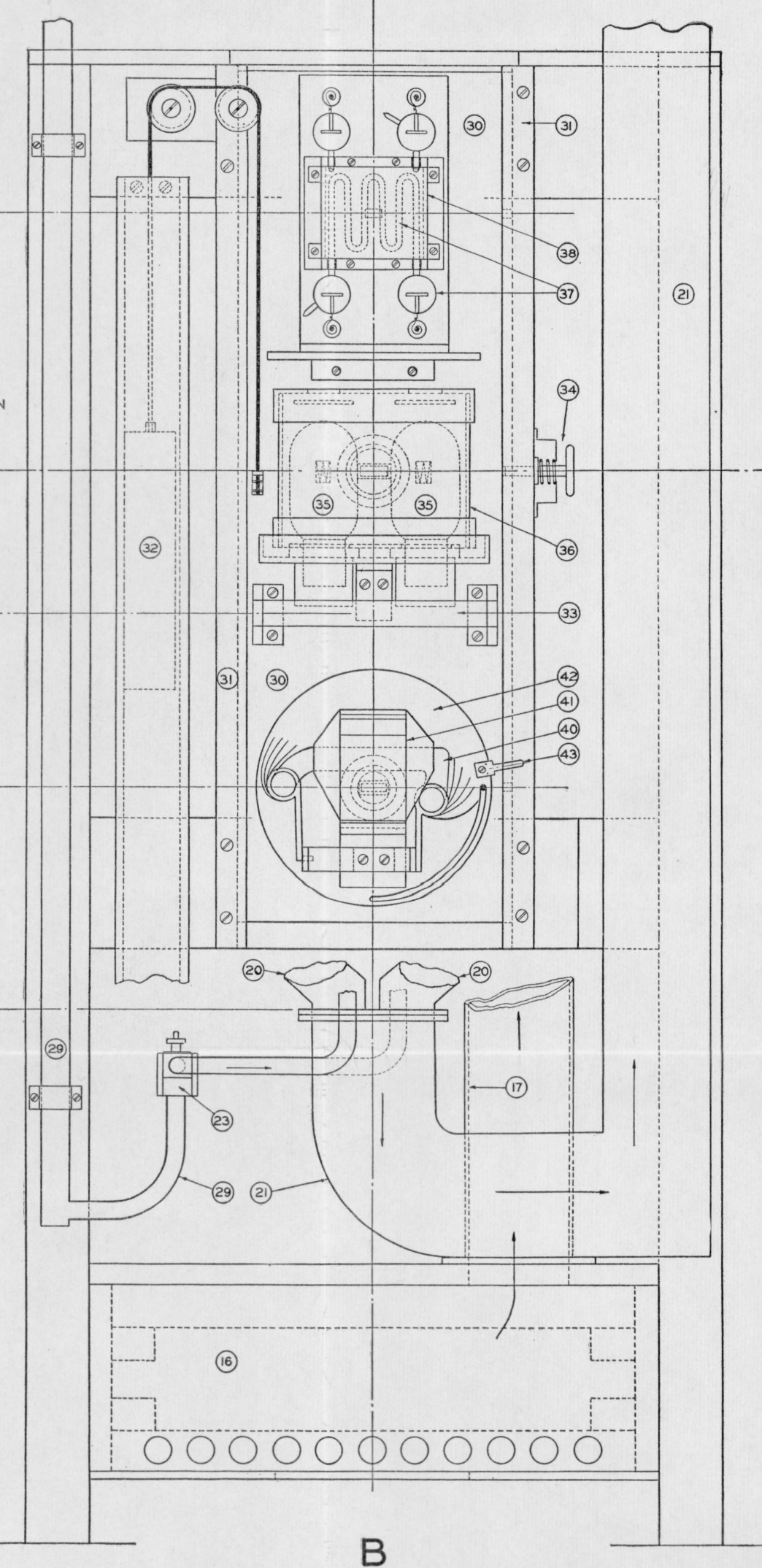

ELEVATION SHOWING:(I) PART OF VENTILATION SYSTEM:

(2) CONSTRUCTION AND MOUNTING OF TRANSMISSSON SOURCES.
(COVER CASES REMOVED FROM HELUMM AND MERCUPY LAMPS)

FIG. 4.-Diagram of illumination apparatus and accessory parts for transmission and reflection measurements

KEY

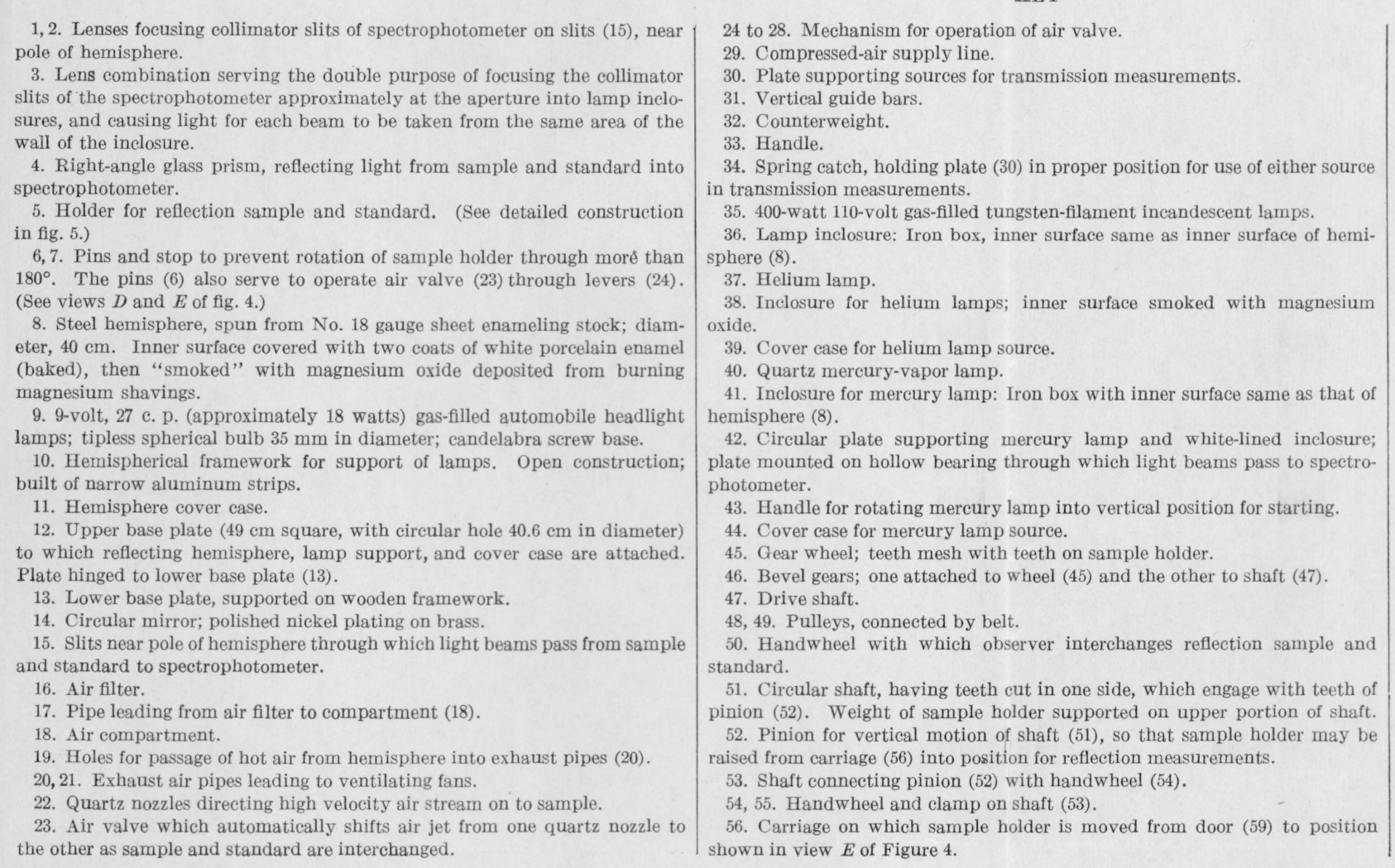

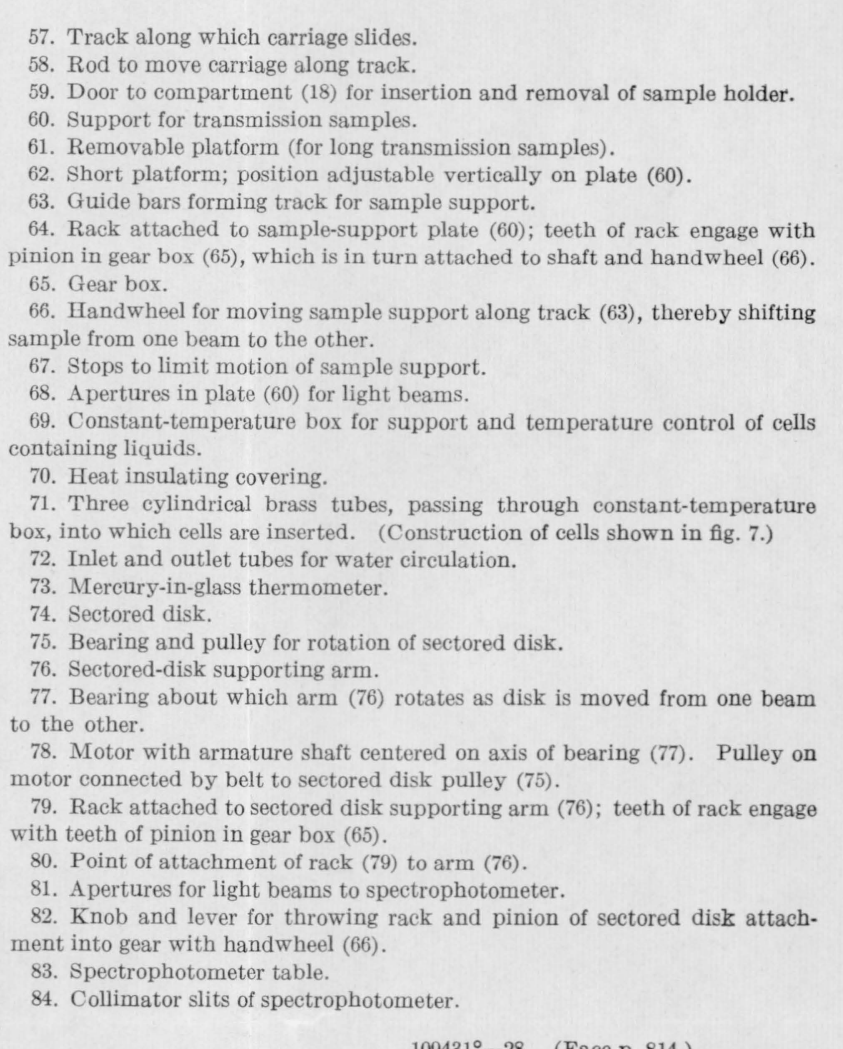


of the biprism faces in a vertical plane which is perpendicular to the plane of double refraction in the Wollaston prism and to the refracting edge of the dispersion prism. The biprism surfaces are made on a wedge-shaped piece of glass, which, with a similar wedge on the collimator lens, prevents the passage to the eye of multiply reflected rays from the optical surfaces.

In the following discussion all directions and displacements are referred to two mutually perpendicular planes, the intersection of which forms the axis of the optical system. One of these planes is conveniently chosen as the vertical plane perpendicular to the plane of double refraction in the Wollaston prism. The other plane coincides with the plane of the paper in the plan of Figure 3 and is hereafter called the horizontal plane.

Consider now a beam of light, homogeneous with respect to wave length and composed of all the elementary ray pencils diverging from various points of the collimator slit $A$; the compound bundle of rays is rendered approximately parallel by the collimator lens, is refracted by the dispersion prism, and falls on the surface of the telescope lens. If the Wollaston prism and the biprism were not in the path of this beam, the rays would be converged by the telescope lens to a focus in the plane of the ocular slit, forming a single image of the collimator slit $A$. Similarly, a single image of slit $B$ would be formed. The two images would be equally displaced horizontally from the vertical reference plane, the displacement corresponding (with reversed sense) to the displacement of the slits $A$ and $B$ from the same vertical plane. With the Wollaston and biprism combination in place, however, the beam from slit $A$ on traversing the Wollaston prism is split into two distinct beams which diverge slightly from each other in a horizontal plane and are each planepolarized with their electrical vibrations in vertical and horizontal directions, respectively. Each of the two beams fills the two inclined faces of the biprism, their effective cross section being determined by the circular field aperture. As a result of the horizontal displacement of slit $A$ from the axis of the optical system, the parallel beam entering the Wollaston prism is itself slightly inclined to the vertical reference plane. The construction of the Wollaston prism is such that the vibrations are vertical in the least refracted (with respect to the vertical plane) of the two beams emerging from the Wollaston prism. Following this least-refracted beam, we find it again divided into two (similarly polarized) beams by further refraction at the two inclined faces of the biprism. These beams finally form two planepolarized images of the slit $A$ in the plane of the ocular slit, the vibrations in each image being vertical. The images are designated by $A_{1} v$ and $A_{2} v$ in Figure 3, and their relative positions in the plane of the ocular slit are indicated in the displaced diagram of this slit 
shown in the lower left corner of Figure 3. For clearness in the representation, various slit images are there shown in different planes; actually, they all lie in the plane of the ocular slit. Going back to the more refracted of the two beams emerging from the Wollaston prism, with vibrations in the horizontal plane, it is found to give rise similarly to the two images $A_{1} h$ and $A_{2} h$ of the slit $A$. In the same manner, light entering slit $B$ finally forms the remaining four slit images shown. The angle of the biprism is chosen, with regard to the separation of the slits $A$ and $B$ and the construction of the Wollaston prism, so that two of the eight images overlap at the ocular slit. The designations $A_{1} v$ and $B_{2} h$ show that one of these images is former by light entering slit $A$ and passing through part 1 of the biprism photometric field. It is plane-polarized with vibrations in a vertical plane. The other image is formed by light from silt $B$ passing face 2 of the biprism and is polarized with vibrations in a horizontal plane.

Considering in like manner all the ray pencils of various wave lengths entering the collimator slits, the different refraction of each homogeneous bundle by the dispersion prism results in eight vertical spectral images of slits $A$ and $B$ being formed in the plane of the ocular slit. Two of these spectra overlap at the ocular slit (as explained above), and a beam of light composed of a small equal range of wave lengths from each spectrum passes through the slit to the eye.

The ocular slit, telescope lens, and attached optical parts are mounted in a tube (the telescope tube) which can be rotated about a horizontal axis through the dispersion prism and the ocular slit thus moved to different parts of the spectrum. A scale attached to the tube indicates the part of the spectrum included by the ocular slit.

Looking through the ocular slit one sees, then, the surface of the biprism uniformly illuminated by monochromatic light of small wave-length range, the mean wave length corresponding to the position of the slit in the spectrum. The field of view is limited by the circular field aperture and divided into two equal parts by the line of intersection of the biprism surfaces. The lights in the two parts of the field are plane-polarized in directions mutually perpendicular to each other, the vibrations in the right half (field 1) being vertical. The brightnesses of the two parts of the field are not, in general, the same. Later discussion will show that this may be the result of unequal conditions of the entrant light beams, but is also caused by an unequal transmission of these beams through the instrument. By rotation of a nicol prism between the eye and the ocular slit the light in either part of the field may be extinguished or the principal plane of transmission of the nicol so orientated that the part fields become matched in brightness. This condition is accompanied by a disappearance of the dividing line between the 
two parts of the field; and, in fact, this circumstance is used as the criterion for the equality of brightnesses. There will obviously be four positions of the principal plane of the nicol, one in each quadrant of the circle, for which this equality of brightnesses may be established. The mounting of the nicol, with its circular scale, may be seen in Figure. 1.

The spectrophotometer is herein used to measure a change in the relative intensities ${ }^{13}$ of the two beams of light entering the instrument, for a given wave length, or small spectral range, included in each beam. The measurement is effected by a comparison of the relative intensities of the beams before and after the change has taken place. In the use of any type of spectrophotometer for this purpose the polarizing action of the prism (or grating) dispersion system makes the reading of the instrument depend not only on the relative intensities of the entrant beams but also - to a lesser extent, usuallyon the states of polarization of the two beams compared. It is therefore of importance to know under what conditions and to what extent a polarized condition of the entrant beams affects the measurements to be made with the Koenig-Martens instrument. In quest of this information, it will be instructive to consider all possible changes in the relative intensities and states of polarization of the two entrant beams on their independent paths from the source to the eye.

In the use of any one of the transmission sources previously described, let it be assumed that there is a difference between the initial intensities and states of polarization of beams 1 and 2 emitted to the spectrophotometer (fig. 3). Likewise, in the use of the hemispherical source, with an exact duplicate of the comparison standard occupying the position of the sample, the possibility of differences in the initial intensities and states of polarization of the two beams is considered. In the general use of the instrument, then, let the initial intensities and states of polarization of beams 1 and 2 be as represented in Figure 9. Here $I_{1}, R_{0}^{\prime}$, and $L^{\prime}$ o represent the initial intensity and component amplitudes, ${ }^{14}$ respectively, of beam 1 , and $I_{2}$, $R^{\prime}{ }_{0}$, and $L_{0}$ are of corresponding import for beam 2. A numerical expression for the polarization is given later in this section of the paper. The definition must obviously imply a given reference frame.

${ }_{13}$ The form of the beams of light entering the spectrophotometer and filling the photometric field of the instrument is determined by the dimensions of the optical system, and a section perpendicular to the axis of the beam varies in size at different positions along the axis. It is convenient to use the expression "intensity of the beam" or beam intensity for the total luminous flux through a cross section of the beam. At the photometric field this section is equal, of course, in shape and area to that of the field itself.

${ }^{14}$ In these considerations of the state of polarization we are not concerned with any phase differences which may possibly exist between the component vibrations in either beam; only changes in the amplitudes of the components are of interest. The word polarization, as used throughout this paper, refers only to this resolution of all the independent vibrations in the beam along two mutually perpendicular directions (which are not necessarily the principal directions of the vibration), giving the resultant magnitude of the amplitude vectors shown in the figures. The square of the vector sum of these components is proportional to the intensity of the beam. 
It is most convenient to resolve the component amplitudes in directions always parallel and perpendicular, respectively, to the vertical reference plane previously defined, so that the directions of the component amplitudes then coincide with the principal directions of vibration in the Wollaston prism.

On passing through the spectrophotometer, both beams traverse optically identical paths. Through the operation of the Fresnel reflection laws at the surfaces of the dispersion prism, however, light with vibrations perpendicular to the refracting edge of the prism (vertical component) is more copiously transmitted than that vibrating parallel thereto. After quadruple subdivision of each beam by the Wollaston and biprism combination (as previously explained), the vertical component amplitude $R$ of the beam passing the ocular

\section{ENTRANT BEAMS}

No.1

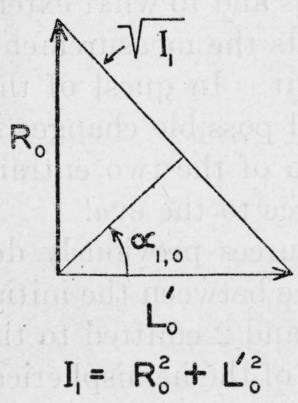

(A)
No. 2

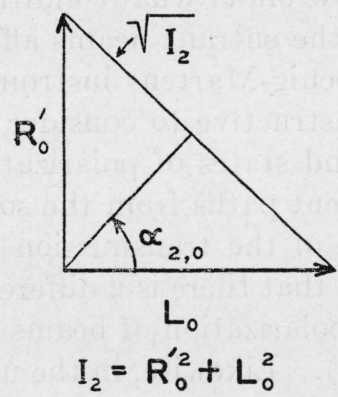

(B)

\section{EMERGENT BEAM AND PHOTOMETRIC FIELD}

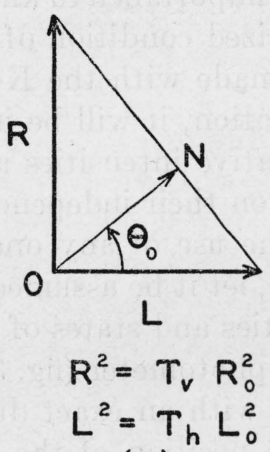

(C)

FIG. 9.-Initial condition of the entrant beams, emergent beam (from ocular siit), and photometric field, before insertion of sample in either beam

slit from field 1 is a certain fraction $\sqrt{T_{\mathrm{v}}}$ of the vertical component amplitude $R_{\mathrm{o}}$ of beam 1 before entering the instrument. Similarly, the horizon tal component amplitude $L$ of the beam passing the ocular slit from field 2 is a fraction $\sqrt{T_{\mathrm{h}}}$ of the horizontal component amplitude $L_{0}$ of beam 2. The components $R^{\prime}{ }_{0}$ and $L^{\prime}{ }_{0}$ of the entrant beams contribute nothing to the intensity of the emergent beam. They are lost in the instrument.

The factors $T_{\mathrm{v}}$ and $\mathrm{T}_{\mathrm{h}}$ are the transmissions of the optical system as a whole (excluding the nicol) for plane-polarized light with vibrations in vertical and horizontal directions, respectively. Thus, $R^{2}=T_{\mathrm{v}}$ $R^{2}{ }_{\mathrm{o}}$ and $\mathrm{L}^{2}=T_{\mathrm{h}} L^{2}{ }_{0}$.

The quantities $R$ and $L$ are proportional, respectively, to the areas of parts 1 and 2 of the photometric field. These areas are equal, however, and hence the brightnesses in the two parts of the field, as 
viewed through the nicol and ocular slit, are proportional, respectively, to the squares of the projections of $R$ and $L$ on the principal plane of transmission of the nicol. Let $O N$ (fig. 9, C) represent the angular position of the principal plane of the nicol, measured (in each quadrant) from the position for extinction of the vertical component amplitude of the emergent beam (position for extinction of light in the right field). Let $\theta_{\mathrm{o}}$ be the position of $O N$ when the two parts of the field are matched in brightness; then

or

$$
R^{2} \sin ^{2} \theta_{0}=L^{2} \cos ^{2} \theta_{0}
$$

$$
\frac{L^{2}}{R^{2}}=\tan ^{2} \theta_{\mathrm{o}}
$$

When the component amplitudes $R_{0}$ and $L_{0}$ of the entrant beams are equal, the angle $\theta_{\mathrm{o}}$ is approximately $39^{\circ}$ (instead of the ideal $45^{\circ}$, for $T_{\mathrm{h}}=T_{\mathrm{v}}$ ); hence

$$
\frac{T_{\mathrm{h}}}{T_{\mathrm{v}}}=\tan ^{2} 39^{\circ}=0.656
$$

The difference between the transmissions of two plane-polarized beams with vibrations in vertical and horizontal directions, respectively, is principally the result of the polarizing action of the dispersion prism. The angle $\theta_{0}$ is not constant, but varies slightly with the wave-length setting.

Now, let the initial intensity and state of polarization of beam 1 be changed. This change may be brought about, for example, by the insertion of a transmission sample in the beam or by the replacement of one of the reflection standards by a sample the spectral reflective properties of which are to be determined. Let $X$ represent the fractional reduction in the intensity of the beam in either case. Then the amplitudes $R_{0}$ and $L^{\prime}{ }_{0}$ are both reduced by the factor $\sqrt{X}$. The quantity $X$ is the transmission of the sample in the first case, and the brightness ratio previously specified (section I) in the second case. The change in the state of polarization of beam 1 may be represented by two factors, $a$ and $b^{\prime}$, attached, respectively, to the components $R_{0}$ and $L_{0}^{\prime}$. These factors are not independent of each other, but are related by the equation

$$
a^{2} R_{0}{ }^{2}+b^{\prime 2} L_{0}^{\prime}{ }^{2}=R_{0}{ }^{2}+L_{0}^{\prime}{ }^{2}
$$

The condition of beams 1 and 2 entering the spectrophotometer (entrant beams), the condition of the combined beams passing through the ocular slit (emergent beam), and the brightness relations in the matched photometric field are now as represented in Figure 10. 
Let $\theta_{1}$ be the angular position of the nicol for the equality of brightnesses in the photometric field; then

or

$$
X a^{2} R^{2} \sin ^{2} \theta_{1}=L^{2} \cos ^{2} \theta_{1}
$$

$$
a^{2} X=\frac{L^{2}}{R^{2}} \cot ^{2} \theta_{1}=\tan ^{2} \theta_{\mathrm{o}} \cot ^{2} \theta_{1}
$$

Now, let the transmission sample be shifted to beam 2, or let the positions of reflection sample and standard be interchanged. Then the intensity of beam 2 is reduced by the fraction $X$, which is the same as in the previous case. The change in the state of polarization of beam 2 may be different than in the previous case but represented in like manner by the factors $a^{\prime}$ and $b$, for which

$$
a^{\prime 2} R^{\prime}{ }^{2}+b^{2} L_{\mathrm{o}}{ }^{2}=R^{\prime 2}+L_{0}{ }^{2}
$$

\section{ENTRANT BEAMS}

No.I

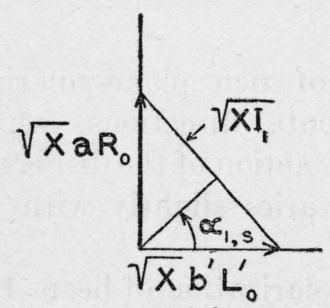

(A)

No. 2

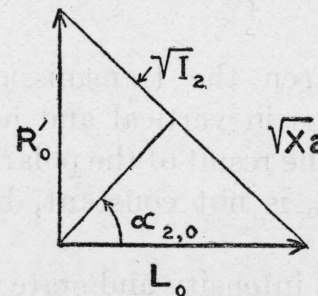

(B)
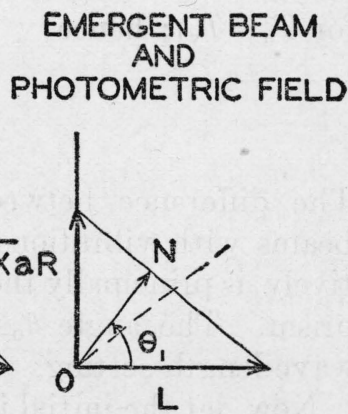

(c)

FIg. 10.-Condition of entrant beams, emergent beam (from ocular slit), and photometric field, after insertion of sample in beam 1

The corresponding conditions of the beams and the photometric field are now as shown in Figure 11. Let $\theta_{2}$ be the new position of the nicol for the equality of brightnesses in the photometric field; then

$$
R^{2} \sin ^{2} \theta_{2}=X b^{2} L^{2} \cos ^{2} \theta_{2}
$$

or

$$
b^{2} X=\frac{R^{2}}{L^{2}} \tan ^{2} \theta_{2}=\cot ^{2} \theta_{0} \tan ^{2} \theta_{2}
$$

By combination of equations (3) and (5) the angle $\theta_{0}$ is eliminated; so that we may write

$$
a b X=\cot \theta_{1} \tan \theta
$$

From equations (3), (5), and (6) it is evident that any initial differences in the beam intensities or any degree of polarization of the light emitted by the source along beams 1 and 2 does not affect the measurement of $X$; any change in the state of polarization, brought 
about by the introduction of a transmission or reflection sample into either beam, must be considered, however, in the measurements. The polarization factors $a$ and $b$ can be expressed in terms of the

\section{ENTRANT BEAMS}

No. 1

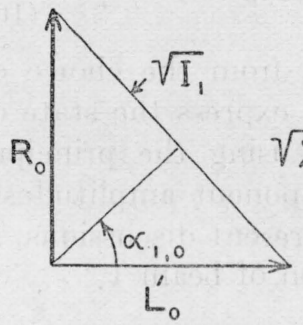

(A)
No. 2

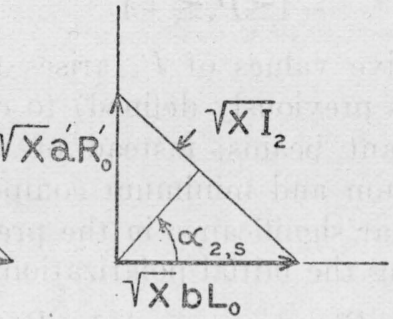

(B)

\section{EMERGENT BEAM AND} PHOTOMETRIC FIELD

Frg. 11.-Condition of entrant beams, emergent beam (from ocular slit), and photometric field, after shifting of sample to beam 2

angles $a_{1,0}, a_{1, \mathrm{~s}}, a_{2,}$ o, and $a_{2, \text { s }}$, of Figures 9,10 , and 11 , and also in terms of the directly measurable fractional polarizations $P_{1,0}, P_{18}$, $P_{2,0}$, and $P_{2, \mathrm{~s}}$. The latter quantities express the states of polarization of beams 1 and 2 before and after the insertion of the sample.

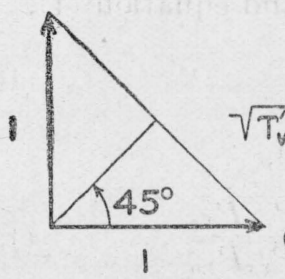

(A)

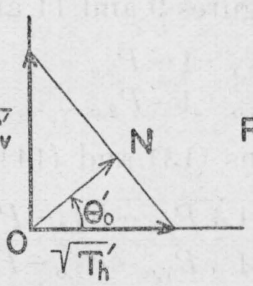

(B)

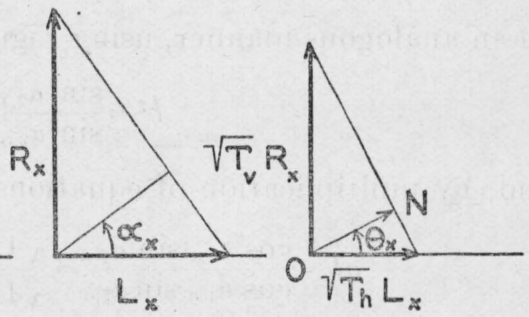

(c)

(D)

FIg. 12.--Representation of the states of polarization of the entrant beam and emergent beam (from ocular slit), when the Koenig-Martens instrument is used as a spectropolarimeter with single central slit. (A) and $(C)$, nonpolarized and polarized entrant beams, respectively; $(B)$ and $(D)$, corresponding emergent beams and conditions in photometric field

Let the subscript "x," as in Figure 12 C, refer to any case of polarization an expression for which is desired. The fractional polarization is defined as follows:

$$
P_{x}=\frac{R_{x}^{2}-L_{x}^{2}}{R_{x}^{2}+L_{x}^{2}}=\cos ^{2} a_{x}-\sin ^{2} a_{x}=\cos 2 a_{x}
$$


Hence

and

$$
\begin{aligned}
& \cos ^{2} a_{x}=\frac{1+P_{x}}{2} \\
& \sin ^{2} a_{x}=\frac{1-P_{x}}{2}
\end{aligned}
$$

$$
-1 \leq P_{\mathrm{x}} \leq+1
$$

The possibility of negative values of $P_{\mathrm{x}}$ arises from the choice of fixed reference planes (as previously defined) to express the state of polarization of the entrant beams, instead of using the principal planes (planes of maximum and minimum component amplitudes), which are not of particular significance in the present discussions.

From Figure 9, showing the initial polarization of beam 1,

$$
\frac{R_{\mathrm{o}}^{2}}{R_{\mathrm{o}}^{2}+L^{\prime 2}{ }_{\mathrm{o}}}=\cos ^{2} a_{1, \mathrm{o}}=\frac{1+P_{1, \mathrm{o}}}{2}
$$

and, from Figure 10, after insertion of the sample,

$$
\frac{a^{2} R_{\mathrm{o}}^{2}}{a^{2} R^{2}{ }_{\mathrm{o}}+b^{\prime 2} L^{\prime 2}{ }_{\mathrm{o}}}=\cos ^{2} a_{1, \mathrm{~s}}=\frac{1+P_{1, \mathrm{~s}}}{2}
$$

Dividing (12) by (11) and using equation (2),

$$
a^{2}=\frac{\cos ^{2} a_{1, \mathrm{~s}}}{\cos ^{2} a_{1, \mathrm{o}}}=\frac{1+P_{1, \mathrm{~s}}}{1+P_{1, \mathrm{o}}}
$$

In an analogous manner, using Figures 9 and 11 and equation (4),

$$
b^{2}=\frac{\sin ^{2} a_{2, \mathrm{~s}}}{\sin ^{2} a_{2, \mathrm{o}}}=\frac{1-P_{2, \mathrm{~s}}}{1-P_{2, \mathrm{o}}}
$$

and, by multiplication of equations (13) and (14),

$$
a b=\frac{\cos a_{1, \mathrm{~s}} \sin a_{2, \mathrm{~s}}}{\cos a_{1, \mathrm{o}} \sin a_{2, \mathrm{o}}}=\frac{\sqrt{1+P_{1, \mathrm{~s}}-P_{2, \mathrm{~s}}-P_{1, \mathrm{~s}} P_{2, \mathrm{~s}}}}{\sqrt{1+P_{1, \mathrm{o}}-P_{2, \mathrm{o}}-P_{1,0} P_{2, \mathrm{o}}}}
$$

When it is permissible to assume that the initial state of polarization of each entrant beam is the same and that the same change in the state of polarization occurs when the sample is inserted in either beam, then

$$
\begin{aligned}
& a_{1, \mathrm{o}}=a_{2, \mathrm{o}}=a_{\mathrm{o}} \\
& a_{1, \mathrm{~s}}=a_{2, \mathrm{~s}}=a_{\mathrm{B}}
\end{aligned}
$$

and equations (13), (14), and (15) become

$$
\begin{aligned}
& a^{2}=\frac{\cos ^{2} a_{\mathrm{s}}}{\cos ^{2} a_{\mathrm{o}}}=\frac{1+P_{\mathrm{s}}}{1+P_{\mathrm{o}}} \\
& b^{2}=\frac{\sin ^{2} a_{\mathrm{s}}}{\sin ^{2} a_{\mathrm{o}}}=\frac{1-P_{\mathrm{s}}}{1-P_{\mathrm{o}}} \\
& a b=\frac{\sin 2 a_{\mathrm{s}}}{\sin 2 a_{\mathrm{o}}}=\frac{\sqrt{1-P_{\mathrm{s}}^{2}}}{\sqrt{1-P_{\mathrm{o}}^{2}}}
\end{aligned}
$$


The polarization factors $a$ and $b$ are, in general, functions of wave length. The measurement of these quantities is discussed in the following section.

\section{USE OF THE INSTRUMENT FOR POLARIZATION MEASUREMENTS}

Although we are not primarily concerned in this paper with the measurement of the polarizing properties of materials, it will be of interest, nevertheless, to extend the preceding discussion to include the general application of the Koenig-Martens instrument to polarization measurements, a purpose for which it is particularly well adapted and has been extensively used by others. ${ }^{15}$ The theory thus available will then be applied to the polarization problem involved in the spectrophotometric measurements.

Let it be required to measure the fractional polarization of a beam of light entering the instrument, the component amplitudes of which are $R_{\mathrm{x}}$ and $L_{\mathrm{x}}$, respectively, as in Figure 12, C. There are two methods of use of the instrument for this purpose, corresponding, respectively, to the use of a single central collimator slit or to the double collimator slit arrangement shown in Figure 3.

To use the central-slit method, let the diaphragm previously described as dividing the collimator slit into two parts, $A$ and $B$, be displaced horizontally until slit $A$ (or slit $B$ ) is centered on the axis of the optical system. The $A$ (or $B$ ) images in the plane of the ocular slit are simultaneously displaced toward the axis of the optical system until the overlapping pair of images $A_{1} h$ and $A_{2} v$ (or $B_{1} h$ and $B_{2} v$ ) are centered on the ocular slit. The vibrations in fields 1 and 2 are now horizontal and vertical, respectively, but the angle $\theta_{\mathbf{x}}$ is still measured from the position of the nicol for the extinction of the vertical component amplitude of the emergent beam (now the position for extinction of light in the left field).

If the instrument is arranged to be movable bodily in a horizontal direction, so that the (now) central slit may occupy either of the positions previously occupied by slits $A$ or $B$ in Figure 3 , the light entering the instrument along either beam 1 or beam 2 may then be analyzed in the manner to be described, both before and after the insertion of the sample. The fractional polarization $P_{\mathrm{x}}$ obtained in each case will be the required value of $P_{1,0}, P_{1,8}, P_{2,0}$, or $P_{2,8}$ in equations (13), (14), and (15).

The absolute intensity of the entrant beam does not enter explicitly into the polarization measurements; hence, the component amplitudes of a nonpolarized entrant beam may each be taken as unity (fig. $12, A)$. Let $\theta^{\prime}{ }_{0}$ be the angular position of the nicol for the equality

1s Woronkoff, G. P., and Pokrowski, G. J., Zeit. f. Physik., 30, p. 129; 1924 (and in later papers by one or both of these authors). Wawilow, S. J., Zeit. f. Physik., 32, p. 721; 1925. 
of brightnesses in the photometric field when the entrant beam is nonpolarized; then (fig. 12, B)

$$
\frac{T_{\mathrm{h}}}{T_{\mathrm{v}}}=\tan ^{2} \theta^{\prime}
$$

where $T_{\mathrm{h}}$ and $T_{\mathrm{v}}$ are again the transmissions of the instrument.

When the entrant beam is polarized, however, with component amplitudes $R_{\mathrm{x}}$ and $L_{\mathrm{x}}$, respectively, the conditions of the entrant beam and the beam transmitted by the ocular slit (emergent beam) are as represented in Figure 12, C and D. For the entrant beam

$$
\frac{L_{\mathrm{x}}}{R_{\mathrm{x}}}=\tan a_{\mathrm{x}}
$$

and for the emergent beam

$$
\sqrt{\frac{T_{\mathrm{h}}}{T_{\mathrm{v}}}} \cdot \frac{L_{\mathrm{x}}}{R_{\mathrm{x}}}=\tan \theta_{\mathrm{x}}
$$

where $\theta_{\mathrm{x}}$ is the new angular position of the nicol for equality of brightnesses in the photometric field. Hence

$$
\tan a_{\mathbf{x}}=\cot \theta^{\prime}{ }_{0} \tan \theta_{\mathbf{x}}
$$

If, now, it be possible to rotate the source of polarization relative to the measuring instrument through an angle of $90^{\circ}$ about the axis of the entrant beam, or, by any other means, to reverse the magnitudes of the component amplitudes $R_{\mathrm{x}}$ and $L_{\mathrm{x}}$ in Figure 12, C, then the angle $\theta^{\prime}{ }_{0}$ may be eliminated from the polarization measurements. In the case of a reflection sample, under the symmetrical (nonpolarized) illumination from the hemispherical source, this reversal of the amplitudes $R_{\mathrm{x}}$ and $L_{\mathrm{x}}$ in the reflected beam is effected simply by a rotation of the sample in its own plane through an angle of $90^{\circ}$ about the prescribed axis.

Let the subscripts " $x$ " and " $y$ " refer, respectively, to the direct and reverse conditions of the entrant beam; then, in the first case,

$$
\tan a_{x}=\cot \theta^{\prime}{ }_{0} \tan \theta_{x}
$$

and in the second case,

$$
\tan \left(90-a_{x}\right)=\cot \theta^{\prime}{ }_{0} \tan \theta_{y}
$$

Dividing one equation by the other,

Hence

$$
\tan ^{2} a_{x}=\tan \theta_{x} \cot \theta_{y}
$$

$$
\begin{aligned}
P_{\mathrm{x}} & =\frac{R_{\mathrm{x}}{ }^{2}-L_{\mathrm{x}}{ }^{2}}{R_{\mathrm{x}}{ }^{2}+L_{\mathrm{x}}^{2}}=\frac{1-\tan ^{2} \alpha_{\mathrm{x}}}{1+\tan ^{2} \alpha_{\mathrm{x}}} \\
& =\frac{\tan ^{2} \theta_{0}^{\prime}-\tan ^{2} \theta_{\mathrm{x}}}{\tan ^{2} \theta_{0}^{\prime}{ }_{0}+\tan ^{2} \theta_{\mathrm{x}}}, \text { by } \\
& =\frac{\tan \theta_{\mathrm{y}}-\tan \theta_{\mathrm{x}}}{\tan \theta_{\mathrm{y}}+\tan \theta_{\mathrm{x}}}, \text { by }
\end{aligned}
$$


In the applications of the Koenig-Martens instrument to polarization measurements it is usually not necessary to replace the double collimator slit by a single central slit. The double-slit arrangement may be regarded as effectually but a splitting and separation of the vertical and horizontal component amplitudes of the single entrant beam analyzed above; for, as previously shown, only the vertical component of beam 1 and the horizontal component of beam 2 contribute to the brightnesses of fields 1 and 2 , respectively. If the source of the polarized light to be measured is extended over a large enough area and is sufficiently uniform so that the state of polarization of each of the entrant beams is the same, the double-slit arrangement is then equivalent to the use of the single central slit.

The component amplitudes $R_{\mathrm{x}}$ and $L_{\mathrm{x}}$ of Figure 12, C may now be regarded as the vertical and horizontal components, respectively, of beams 1 and 2, and equations (24) and (25) apply without modification to this case. The intensities of the polarized entrant beams need not be the same, provided that the angle $\theta^{\prime}$ o (now the angle $\theta_{0}$ of equation (1)) is determined for the same relative intensities of two nonpolarized entrant beams or that the method of rotating the sample through $90^{\circ}$ is used.

The required conditions for the measurement of the polarization of the transmitted or reflected light may be approximately realized with the apparatus herein described by placing two specimens of the same samples orientated in the same way in each beam. It must be assumed, however, that the two specimens are identical, so that the state of polarization of each entrant beam is the same.

\section{USE OF THE INSTRUMENT FOR REFLECTION AND TRANSMISSION MEASUREMENTS}

(a) The Polarization Problem: Simplified Formulas.--In the preceding discussion the general theory of the use of the instrument in the measurement of the relative intensities and states of polarization of the entrant beams has been considered. This theory may now be simplified in its application to the measurement of the particular transmissive and reflective properties previously specified. It will be shown that a quantitative consideration of the polarization factors may always be avoided in the measurement of $X$ with the present illumination apparatus and method of use of the instrument.

The construction of the light sources herein described is such that one would expect to find very little polarization of the light emitted directly to the spectrophotometer. Moreover, any polarization present would be practically the same for all wave lengths. To meet the conditions required in the measurement of the fractional polarization of the light from these sources, it is more convenient and wholly ade$109431^{\circ}-28-3$ 
quate for the purpose to use the Martens ${ }^{16}$ polarization photometer (with central aperture) instead of the spectrophotometer.

The general theory and optical principles involved in the construction, operation, and use of this instrument for both relative intensity and polarization measurements are the same as described on the preceding pages for the Koenig-Martens spectrophotometer. The essential difference in the construction of the two instruments lies in the absence of a dispersion system in the Martens photometer. When used with central aperture the instrument measures an average polarization for all wave lengths contained in the entrant beam. The angle $\theta^{\prime}{ }_{0}$ (equation (19)) for this instrument is practically $45^{\circ}$; so that equations (24) and (25) both reduce, in this case, to the single equation

$$
P_{\mathrm{x}}=\frac{1-\tan ^{2} \theta_{\mathrm{x}}}{1+\tan ^{2} \theta_{\mathrm{x}}}=\frac{\cos ^{2} \theta_{\mathrm{x}}-\sin ^{2} \theta_{\mathrm{x}}}{\cos ^{2} \theta_{\mathrm{x}}+\sin ^{2} \theta_{\mathrm{x}}}=\cos 2 \theta_{\mathrm{x}}
$$

Using the Martens photometer, then, in the same manner as described for the spectrophotometer with central slit, it has been found that the initial polarization of the light emitted to the spectrophotometer from the tungsten, mercury-vapor, and helium lamp sources is in each case less than 1 per cent. Under the symmetrical illumination from the hemispherical source, there is no appreciable polarization of the normally reflected light from a sample having no structural regularities differing markedly in mutually perpendicular directions parallel to its surface. The comparison standard used is a material of this type. (See section V,2.)

In the case of some reflection samples, however, including certain types of textile materials, such as velvets and plushes, there is, in general, an appreciable polarization of the normally reflected light even under completely diffused nonpolarized illumination.

To illustrate the case, the spectral polarization curves of four textile samples have been obtained with the Koenig-Martens instrument, used as a spectropolarimeter with double collimator slit. The curves are shown in Figure 13. In accordance with the theory of polarization measurements, two (square) specimens of each sample were mounted on heavy cardboard and inserted, one in each beam, in the regular position of the sample and standard. The photometric part fields of the instrument were matched with the specimens in the 0 and $90^{\circ}$ positions (" $x$ " and " $y$ " positions), giving the angles $\theta_{\mathrm{x}}$ and $\theta_{\mathrm{y}}$, respectively. The fractional polarization was then computed by equation (25). The initial (or " $\mathrm{x}$ ") orientation of each sample was chosen to give the maximum positive polarization of the entrant beams as referred to the vertical plane, so that the data would serve as an illustration of the magnitude of the polarization effect

${ }_{16}$ Martens, F. F., Phys. Zeit. 1, pp. 299-303; 1900. 
which may be obtained in practical cases. In these measurements it is assumed that the state of polarization of the light reflected from each of the specimens is the same. This condition depends, of course, on the uniformity of the two specimens and is fulfilled to a sufficient approximation for these purposes, for an interchange of the specimens from one beam to the other had but slight effect on the angles $\theta_{\mathrm{x}}$ and $\theta_{\mathrm{y}}$.

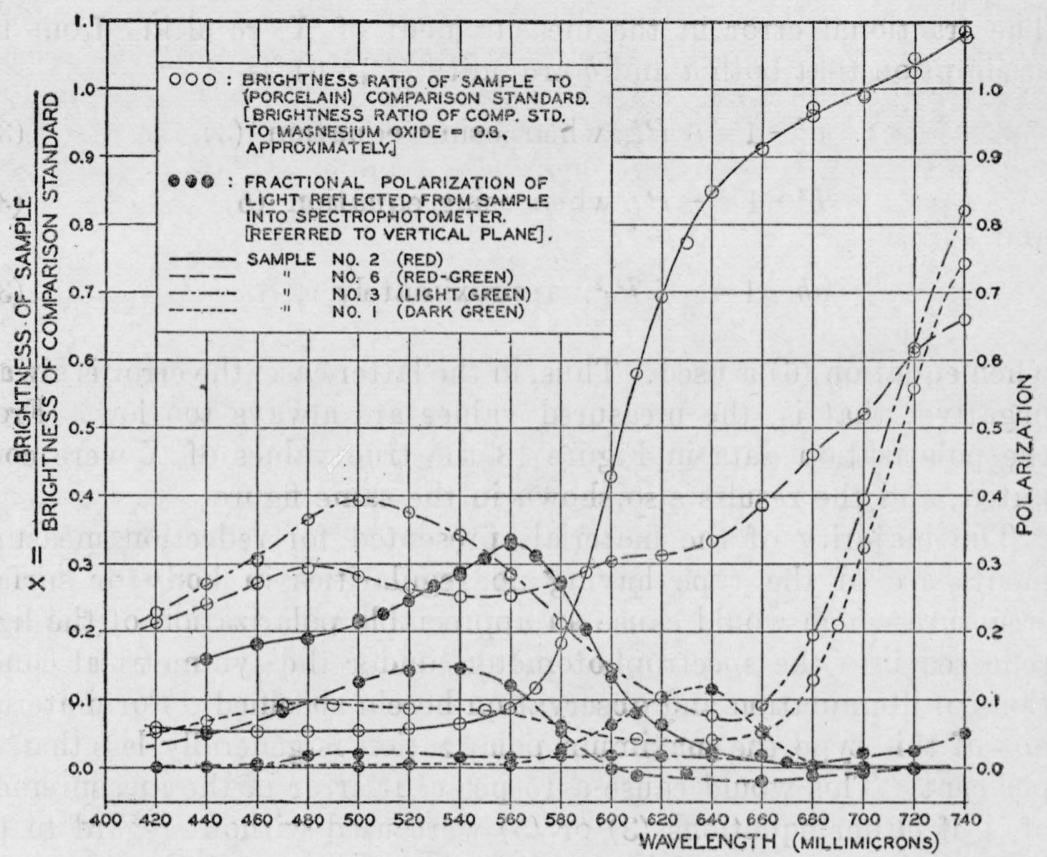

FIG. 13.-Reflection and polarization measurements made with the KoenigMartens spectrophotometer, under the specified conditions of illumination and observation

Samples were chosen from certain textile materials in order to illustrate, particularly, the degree of polarization of the normally reflected light under nearly completely diffused nonpolarized illumination. The orientation of the sample (in its own plane) was chosen to obtain the maximum polarization with reference to the vertical plane.

Samples Nos. 2 and 3 are cotton back, silk cut-pile fabrics (velvets). No. 1 is a warp face sateen silk. No. 6 is a plain weave broadcloth with a silk warp (dyed green) and a rayon filling (dyed pink).

Replacing one of the specimens by the comparison standard, the values of $a b X$ for various wave lengths were obtained in the regular way (described later in this section) as the product $\cot \theta_{1} \tan \theta_{2}$ by equation (6).

It is now of interest to determine the difference between the measured quantity $a b X$ and the value of $X$ itself. Having shown that the initial polarization of the light emitted to the spectrophotometer by the hemispherical source (light reflected from comparison standard) and by the transmission sources is very small, equations 
(16), (17), and (18) may then be further simplified by placing $P_{0}=0$. Thus

$$
\begin{aligned}
& a^{2}=2 \cos ^{2} \alpha_{\mathrm{s}}=1+P_{\mathrm{s}} \\
& b^{2}=2 \sin ^{2} \alpha_{\mathrm{s}}=1-P_{\mathrm{s}} \\
& a b=\sin 2 \alpha_{\mathrm{s}}=\sqrt{1-P_{\mathrm{s}}^{2}}=1-\frac{1}{2} P_{\mathrm{s}}^{2}, \text { approximately }
\end{aligned}
$$

The fractional error in the measurement of $X$, resulting from the assumption that both $a$ and $b$ are unity $\left(P_{\mathrm{s}}=0\right)$, is

and

$$
\begin{aligned}
& a^{2}-1=+P_{\mathrm{s}}, \text { when using equation } \\
& b^{2}-1=-P_{\mathrm{s}} \text {, when using equation }
\end{aligned}
$$

$$
a b-1=-\frac{1}{2} P_{\mathrm{s}}^{2}, \text { approximately }
$$

when equation (6) is used. Thus, in the latter case, the error is always negative; that is, the measured values are always too low. From the polarization data in Figure 13 the true values of $X$ were computed, and the results also shown in the same figure.

The majority of the materials presented for reflection measurements are of the type having no regularities in body or surface structure which would cause an appreciable polarization of the light reflected in to the spectrophotometer, under the symmetrical conditions of illumination and observation herein specified. For materials not of this type the maximum polarization is generally less than 15 per cent. This would cause a 15 per cent error in the measurement of $X$ if either equations (3) or (5) were used without regard to the polarization factors. Using equation (6), however, in this way, the error is only 1 per cent. Sample No. 2 (fig. 13) represents an extreme case. The polarization of 33 per cent at wave length $560 \mathrm{~m} \mu$ would cause an error of 5.5 per cent in the measurement of $X$, if the polarization of the reflected light is not taken into consideration. ${ }^{17}$

As may be seen from the curves of Figure 13, the polarization is generally higher in the spectral regions wherein the material strongly absorbs the incident light. Superposed on this effect, however, there appears to be a general decrease in the polarization for shorter wave lengths.

The polarization errors in the measurement of $X$ may be reduced to negligible proportions by proper orientation of the sample in its own plane, for it is always possible in this way to reduce the polari-

${ }^{17}$ It may be noted that the polarization errors herein considered are not particularly inherent in the use of the Koenig-Martens spectrophotometer. Thus, in the case of another type of spectrophotometer containing no polarizing parts whatever other than the dispersion prism, a 33 per cent polarization of the light reflected from the sample would cause an error of 10 per cent in the measurement of $X$. Such a spectrophotometer is part of the equipment briefly described later in section $\mathrm{V}, 2$. 
zation factors $a$ and $b$ each to unity, leaving the initial state of polarization of the entrant beam unchanged. An inspection of the sample structure will usually be sufficient to determine the reference plane of maximum polarization (positive or negative) under the symmetrical hemispherical illumination, and the sample may then be cut and mounted in the sample holder so that this plane of maximum polarization makes an angle of approximately $45^{\circ}$ with the vertical reference plane previously defined (through the optical axis of the spectrophotometer). The component amplitudes of the entrant beam are then approximately the same, and since the initial polarization of the beam is practically zero, the required condition is fulfilled. If the initial polarization of the beam was not negligible, however, a different orientation of the sample would be required. In the operation of interchanging the positions of the sample and standard for a reflection measurement, the sample itself rotates through $180^{\circ}$, so that its orientation with respect to the spectrophotometer is, under the symmetrical illumination, effectually unchanged.

In transmission measurements we are usually not troubled with any polarization introduced by the sample itself. If the incident beam is appreciably polarized, however, a strained-glass sample or an optically active solution may possibly so alter the state of polarization as to introduce an appreciable error into the transmission measurements. It is well, therefore, that the initial polarization of the beams incident on the sample be small, as is the case with the sources herein employed.

In conclusion, it may be stated that in all the transmission and reflection measurements to be made with the equipment herein described, the polarization factors may be either neglected or eliminated by proper procedure in the measurements. Hence the previous formulas for $X$ reduce simply to

$$
\begin{aligned}
& X=\tan ^{2} \theta_{0} \cot ^{2} \theta_{1} \\
& X=\cot ^{2} \theta_{0} \tan ^{2} \theta_{2} \\
& X=\cot \theta_{1} \tan \theta_{2}
\end{aligned}
$$

(b) Propagation of Error Through the Formulas.- It is sometimes desirable to know the precision with which the value of $X$ is determined from a set of readings of the angles of match when using any one of the equations (33), (34), and (35). For this purpose let $Z_{1}, Z_{2}$, and $Z$ be the fractional probable errors ${ }^{18}$ in the computed values of $X$ by equations (33), (34), and (35), respectively; and let $p_{0}, p_{1}$, and $p_{2}$ be the probable errors in the determination of the corresponding angles $\theta_{0}, \theta_{1}$, and $\theta_{2}$. Then, in accordance with the general theory of the propagation of error, ${ }^{19}$ the following relations 18 The fractional probable error is here defined as the ratio of the probable error in $X$ to the value of $X$
itself.

10 Merriman, Method of Least Squares, 8th ed., p. 79.

$109431^{\circ}-28-4$ 
may be derived (in which the probable errors are expressed in radians):

$$
\begin{aligned}
Z_{1}^{2} & =16\left(p_{1}^{2} \csc ^{2} 2 \theta_{1}+p_{\mathrm{o}}{ }^{2} \csc ^{2} 2 \theta_{\mathrm{o}}\right) \\
Z_{2}^{2} & =16\left(p_{2}^{2} \csc ^{2} 2 \theta_{2}+p_{\mathrm{o}}{ }^{2} \csc ^{2} 2 \theta_{\mathrm{o}}\right) \\
Z^{2} & =4\left(p_{1}^{2} \csc ^{2} 2 \theta_{1}+p_{2}^{2} \csc ^{2} 2 \theta_{2}\right)
\end{aligned}
$$

Let $\theta_{0}, \theta_{1}$, and $\theta_{2}$ be each the mean of $n$ settings of the angle of match. Let $\Sigma \Delta \theta_{0}, \Sigma \Delta \theta_{1}$, and $\Sigma \Delta \theta_{2}$ be the sum of the $n$ residuals of $\theta_{0}, \theta_{1}$, and $\theta_{2}$, respectively, these residuals being taken without regard to algebraic sign and expressed in circular degrees. The relation between the probable error of the mean of the observed angles and the sum of the residuals is given by the approximate formula ${ }^{20}$

$$
p=\frac{0.8453 \Sigma \Delta \theta}{n \sqrt{n-1}}
$$

In routine spectrophotometric measurements, 10 settings are usually made of each angle of match. Placing $n=10$ in equation (39) and remembering that $p$ in equations (36), (37), and (38) must be expressed in radians, the following formulas are then obtained:

$$
\begin{aligned}
& Z_{1}=0.002 \sqrt{\frac{\left[\Sigma \Delta \theta_{0}\right]^{2}}{\sin ^{2} 2 \theta_{0}}+\frac{\left[\Sigma \Delta \theta_{1}\right]^{2}}{\sin ^{2} 2 \theta_{1}}} \\
& Z_{2}=0.002 \sqrt{\frac{\left[\Sigma \Delta \theta_{0}\right]^{2}}{\sin ^{2} 2 \theta_{0}}+\frac{\left[\Sigma \Delta \theta_{2}\right]^{2}}{\sin ^{2} 2 \theta_{2}}} \\
& Z=0.001 \sqrt{\frac{\left[\Sigma \Delta \theta_{1}\right]^{2}}{\sin ^{2} 2 \theta_{1}}+\frac{\left[\Sigma \Delta \theta_{2}\right]^{2}}{\sin ^{2} 2 \theta_{2}}}
\end{aligned}
$$

A useful graph for the computation of the probable error by any one of the equations (40), (41), and (42) has been previously published, ${ }^{21}$ but is reproduced in the present paper in Figure 14. The legend on this graph clearly explains its use with equations (35) and (42). The symbols $X$ and $Z$ are replaced by $T$ and $(P . E .)_{T} / T$, respectively, on the graph. Its use with equations (40) and (41) is obviously the same.

(c) Choice of Formula for Routine Work.-It is evident that two different methods of use of the instrument are available for the measurement of $X$. In the first method the two parts of the field are matched with the sample in either beam 1 or beam 2 , and again with both beams clear. Equation (33) is used in the first case and equation (34) in the second case. In the second method the two parts of the field are first matched with the sample in (say) beam 1, then again with the sample in beam 2 , and equation (35) is used.

${ }^{20}$ Smithsonian Physical Tables, 2d rev. ed., p. 59; 1920.

21 See paper by Priest, McNicholas, and Frehafer on "Some tests of the precision and reliability of measurements of spectral transmission by the Koenig-Martens spectrophotometer," J. O. S. and R. S. I., p. $211 ; 1924$. 
The second method is generally applied in all work with the instrument, chiefly for the following reasons:

1. The possibility of polarization errors are very much reduced, and we may assume that for all ordinary samples no appreciable polarization errors are present in the measurement of $X$ when equation (35) is used.

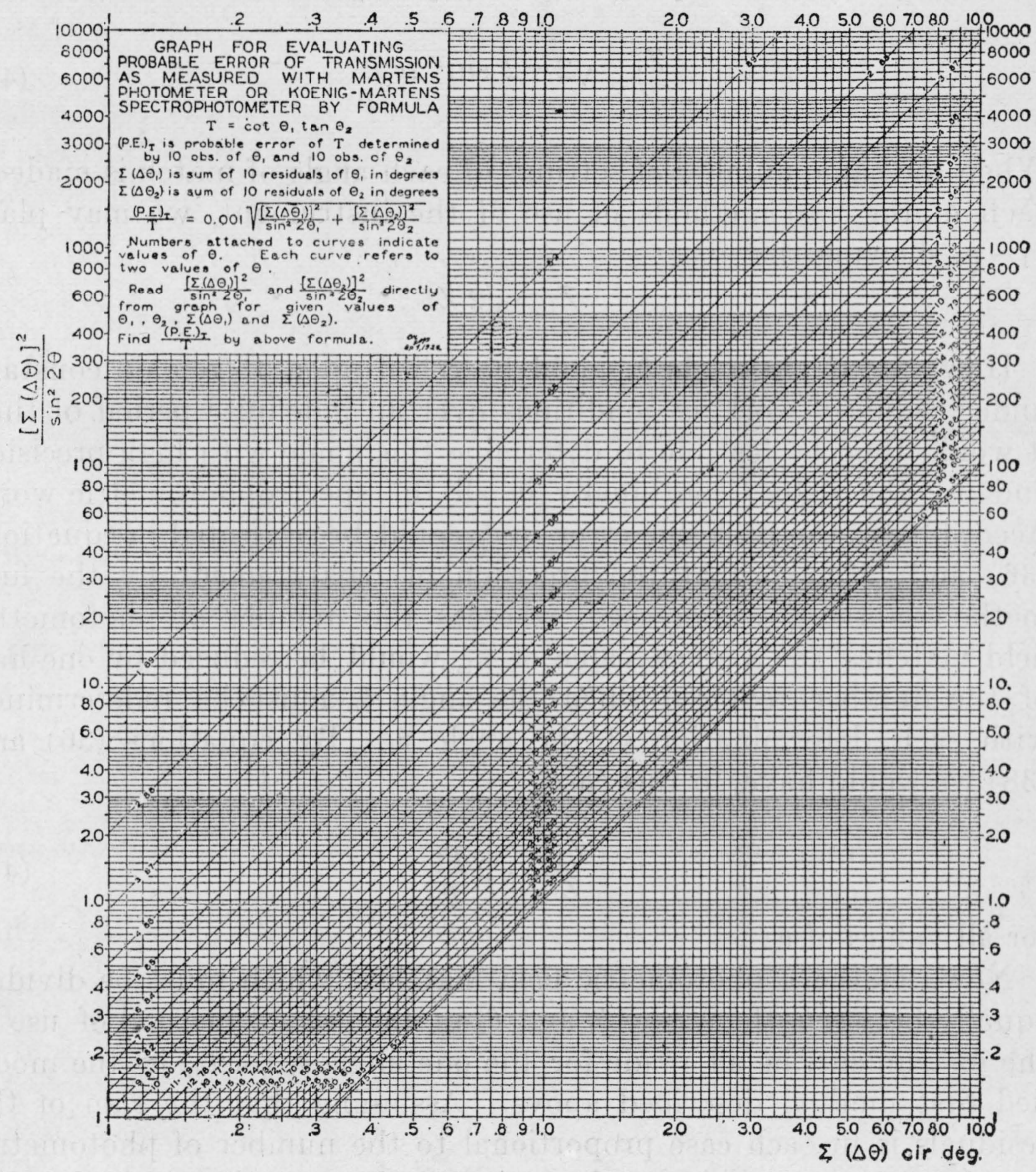

Fig. 14.-Graph for the evaluation of probable errors

2. In the second method the field brightness at match is nearly the same for both positions of the sample. This is an important consideration in the measurement of low values of $X$, as it avoids eye strain and consequent loss in photometric precision which would result from alternate settings at widely different field brightnesses.

3. A consideration of the probable errors in $X$ shows that the precision with which the value of $X$ is determined from a set of readings of the angles of match is different for each of the equations 
(33), (34), and (35), and that a greater precision of measurement is generally obtained in the second method of use of the instrument.

In the following comparison of the two methods of use of the instrument we are justified in making the following approximations in the preceding formulas for the probable errors: Let $p_{0}=p_{2}=p_{1}$ in equations (36) and (37), and let $p_{2}=p_{1}=p$ in equation (38). Place $\theta_{0}=45^{\circ}$, so that $\theta_{2}=90-\theta_{1}$ and $Z_{2}=Z_{1}$. Then

$$
\frac{Z_{1}}{Z}=\sqrt{2+2 \sin ^{2} 2 \theta} \frac{p_{1}}{p}
$$

When the same number of settings of each angle of match is made in each of the two methods of use of the instrument, we may place $p_{1}=p$, showing that

$$
Z_{1}>\sqrt{2} Z
$$

However, if the angle $\theta_{0}$ could be relied upon to remain constant under ordinary working conditions over an indefinite period of time it would then be feasible to determine this angle with high precision and use the values continually in all the spectrophotometric work. Accordingly, the probable error in $\theta_{\mathbf{o}}$ could be neglected in equations (36) and (37), so that the precision of measurement by the first method is thereby increased, whereas the number of photometric field matches, for a given value of $p_{1}$, would be reduced by one-half of the number required when the angle $\theta_{0}$ must be redetermined with each determination of the angle $\theta_{1}$. By equations (36) and (38) we would have, in this case,

$$
\frac{Z_{1}}{Z}=\sqrt{2} \frac{p_{1}}{p}
$$

for all values of $\theta_{1}$.

Now, let the $n$ photometric field matches, which must be divided equally between the angles $\theta_{1}$ and $\theta_{2}$ in the second method of use of the instrument, be all made for the one angle $\theta_{1}\left(\right.$ or $\left.\theta_{2}\right)$ in the modified first method described above. Assuming that the sum of the residuals is in each case proportional to the number of photometric matches, we may evaluate the ratio $p_{1} / p$ by equation (39), showing that

$$
\frac{p_{1}}{p}=\frac{1}{\sqrt{2}} \cdot \frac{\sqrt{n-1}}{\sqrt{n-1 / 2}}
$$

Hence, in this case, $Z_{1}=Z$, approximately, when $n$ is fairly large, say about 10 or 20 .

Whereas some advantages might be derived from the use of equations (33) or (34) with the Martens photometer under certain experimental conditions, it is probable that the modified first method would 
not be satisfactory and reliable for general use in routine spectrophotometric measurements with the equipment herein described. As previously mentioned, the angle $\theta_{0}$ varies slightly throughout the spectrum and would be subject to change with the deposition of dust or with the formation of slight diffusing films on the optical surfaces, particularly those surfaces outside of the instrument, where the beams of light to the spectrophotometer are completely separated.

(d) Use of One Quadrant of the Circular Scale on the Nicot Mounting.-As previously stated, angles of match can be obtained in all four quadrants of the circular scale when the sample is placed in either beam. In order to eliminate all errors in the reading of these angles, such as may be caused by eccentricities of the circular scale and vernier of the nicol mounting, it is necessary to average settings made in all four quadrants of the circle. The use of four quadrants instead of one slightly complicates the experimental procedure, with the result that more time is consumed in the measurements. In routine work, therefore, it is desirable to avoid this complication, if possible.

To investigate the relative importance of these errors, let the true angular position of the principal plane of transmission $O N$ of the nicol be given by the (imaginary) $\theta$ scale shown in Figure 15. This scale is fixed in position relative to the instrument and is accurately centered on the axis of rotation of the nicol. Angles are measured in each quadrant from the position of $O N$ for the extinction of light in the right field. Assuming that the vibrations in the plane-polarized beams entering the nicol from fields 1 and 2 are exactly at right angles to each other, then the angular position of the nicol for extinction of light in the left field is exactly $90^{\circ}$ on the $\theta$ scale.

Let the $\phi$ scale be the circular scale actually engraved on the nicol mounting. The following assumptions are made in regard to this scale:

1. The engraving of the scale is accurate, all divisions being exactly $1^{\circ}$ apart.

2. The center $O^{\prime}$ of the graduated circle is slightly eccentric with the center $O$ of the $\theta$ scale. Hence $O^{\prime}$ rotates about $O$ with rotation of the nicol.

3. When the nicol is set in position for extinction of the right field (zero position, as in fig. 15), there is a small angular displacement of the whole $\phi$ scale relative to the $\theta$ scale, and a displacement of the vernier so that its zero line may not coincide with either the zero or $90^{\circ}$ marks of the $\phi$ and $\theta$ scales. The $\phi$ scale rotates with the nicol, the (single) vernier being attached to the body of the instrument. The angular displacements of both the index and the $\phi$ scale relative to the $\theta$ scale cause errors in the reading of $\theta$ which are constant (numerically) over the whole circle. In the discussion of these 
errors, however, we can regard the position of the index as exactly coincident with the $90^{\circ}$ mark on the $\theta$ scale and include both displacements in a single rotation of the $\phi$ scale.

The errors in the reading of the angular positions of the nicol are caused, then, by the combined effect of the linear and rotary displacements of the $\phi$ scale relative to the $\theta$ scale.

It is evident that there is only one angle of match, $\theta$, for which the readings in opposite quadrants of the $\phi$ scale are exactly $180^{\circ}$ apart. At this value of $\theta$ the center of the $\phi$ scale lies on the line connecting the zero of the vernier with the center of the $\theta$ scale. For all other angles of match the reading in one quadrant will be as much too large

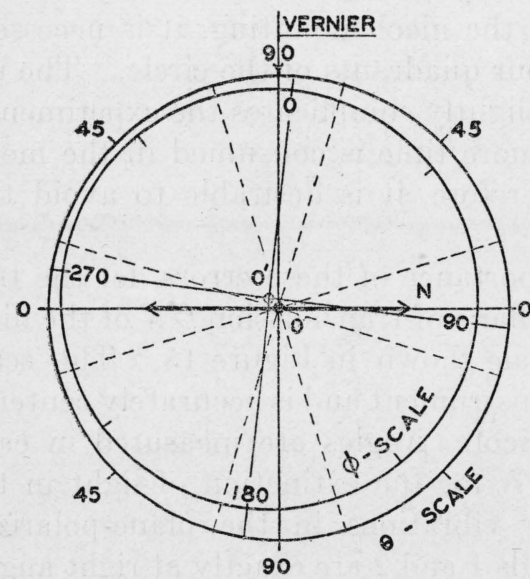

(A)

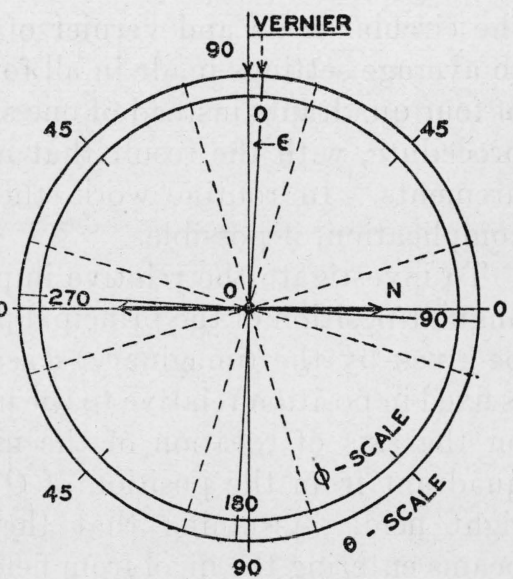

(B)

FIG. 15.-Illustration of errors in the circular scale on nicol mounting (Koenig-Martens spectrophotometer)

Double arrow $O N$ represents principal plane of transmission of nicol. $O$ indicates axis of rotation of nicol. $\Theta$ scale (imaginary) gives true angular position of $O N$. $\Phi$ scale is the circular scale actually engraved on nicol mounting. ( $A$ ) shows combined effect of eccentricity of centers and rotatory displacement of $\Phi$ scale relative to $\Theta$ scale. $(B)$ shows rotatory displacement only. The dotted lines indicate positions of $O N$ for a photometric field match in each of the four quad. rants, and corresponding angles read with the vernier.

as the reading in the opposite quadrant is too small, so that the error resulting from an eccentricity of the centers is eliminated by averaging the two readings in opposite quadrants. The error caused by a pure rotation of the $\phi$ scale relative to the $\theta$ scale is, on the other hand, eliminated by proper averaging of readings in adjacent quadrants. Let subscripts I, II, III, and IV denote readings made in the first, second, third, and fourth quadrants, respectively. Then

$$
2 \theta=\frac{\phi_{I}+\left(\phi_{I I I}-180\right)}{2}+\frac{\left(180-\phi_{\text {II }}\right)+\left(360-\phi_{\text {IV }}\right)}{2}
$$

or

$$
4 \theta=360+\phi_{\mathrm{I}}-\phi_{\mathrm{II}}+\phi_{\mathrm{III}}-\phi_{\mathrm{IV}}
$$


It is evident that the same small angular increment added to each value of $\phi$ in the above formula leaves the value of $\theta$ unchanged. Thus, by combining angles of match made in all four quadrants in the above manner, all errors in the reading of the value of $\theta$ on the $\phi$ scale are eliminated.

The amount of the eccentricity and rotary displacement of the $\phi$ scale relative to the true $\theta$ scale may be determined from readings of the angles of match in each of the four quadrants of the circle, when a low transmission sample (for instance) is placed alternately in each beam. The $\theta$ values will be the same in adjacent quadrants, but the readings on the $\phi$ scale will generally not be equidistant from the $0,90,180$, or $270^{\circ}$ marks. Let the subscripts 1 and 2 refer to the positions of the sample in beams 1 and 2 , respectively. The angular differences between the $0,90,180$, and $270^{\circ}$ marks on the $\phi$ scale and the corresponding positions on the $\theta$ scale are denoted by the general symbol $[\theta-\phi]$ and given by the following equations:

$$
\begin{gathered}
{[\theta-\phi]_{\phi=0}=180-\frac{1}{2}\left(\phi_{2, \mathrm{IV}}+\phi_{2, \mathrm{I}}\right)} \\
{[\theta-\phi]_{\phi=90}=90-\frac{1}{2}\left(\phi_{1, \mathrm{I}}+\phi_{1, \mathrm{II}}\right)} \\
{[\theta-\phi]_{\phi=180}=180-\frac{1}{2}\left(\phi_{2, \mathrm{II}}+\phi_{2, \mathrm{III}}\right)} \\
{[\theta-\phi]_{\phi=270}=270-\frac{1}{2}\left(\phi_{1, \mathrm{III}}+\phi_{1, \mathrm{IV}}\right)}
\end{gathered}
$$

These differences are the result of both sources of error mentioned above and will not generally have the same value. (Fig. 15, A.) Let $\epsilon$ be the average of the four values of $[\theta-\phi]$ thus obtained. Then $\epsilon$ represents the rotational displacement of the scale as a whole, and the residuals are an indication of the eccentricity of the center. If the eccentricity of the $\phi$ scale is negligible or zero, however, the $[\theta-\phi]$ differences are the same for all parts of the scale. In this case (the sample being stationary in either beam)

$$
4 \epsilon=720-\phi_{\mathrm{I}}-\phi_{\mathrm{II}}-\phi_{\mathrm{III}}-\phi_{\mathrm{IV}}
$$

True values of $\theta$ can then be obtained by applying the constant correction $\epsilon$ to the readings of $\phi$, as made in one quadrant only, or by adjusting the vernier into coincidence with the zero mark on the $\phi$ scale when the nicol is set for the extinction of light in the right field.

To test the accuracy of the scale on the particular instrument herein used, angles of match have been made in all four quadrants in the measurement of the transmission of the 1 and 10 per cent sectored disks. The mounting of the disks has been described, and their use 
with the instrument will be further discussed in section $V, 1$. The $[\theta-\phi]$ differences in equations $(46),(47),(48)$, and (49) were found to be practically the same for this particular scale, and the value of $\epsilon$ was $0.24^{\circ}$. In Table 1 , columns $3,4,5$, and 6 , the transmissions are recorded as computed from the corrected angles of match, using each quadrant separately. In column 8 the transmissions are computed from the averaged angle as given by equation (45). The purpose of this table is to show that it is not necessary to make readings in more than one quadrant of the circular scale in routine spectrophotometric work with this particular instrument. Errors introduced by neglecting the small correction to the scale readings are reduced by use of equation (35) instead of either of equations (33) or (34), for the angles of match are then more nearly equally distant from $45^{\circ}$, and the same small increment added to both $\theta_{1}$ and $\theta_{2}$ causes counteractive changes in the cotangent and tangent. If the scale correction $\epsilon$ were neglected in the computation of the transmissions in column 3 of Table 1 , the values obtained would be on the average about 1.8 per cent lower than the values in column 8 . In the measurements of the 10 per cent sectored disk the values would be lower by only 0.4 per cent. By care in the adjustment of the vernier the above errors may be further reduced. An error of $0.2^{\circ}$ is of no importance unless very low values of $X$ are being measured, so that $\theta_{1}$ and $\theta_{2}$ are close to 90 or $0^{\circ}$, respectively. Readings in these parts of the scale are therefore avoided by use of the sectored-disk attachment.

In routine work with the instrument, then, readings of the angle of match are made only in the first quadrant of the circular scale. An occasional check is made on the adjustment of the vernier.

TABLE 1.-Transmission measurements with the Koenig-Martens spectrophotometer, using four quadrants of the circular scale on the nicol mounting

\begin{tabular}{|c|c|c|c|c|c|c|c|}
\hline Sample & $\begin{array}{l}\text { Wave } \\
\text { length }\end{array}$ & \multicolumn{5}{|c|}{$\begin{array}{l}T=\cot \left(360-\phi_{1}-\epsilon\right) \tan \left(360-\phi_{2}-\epsilon\right) \text { (for quadrants I } \\
\text { and IV) } \\
T=\cot \left(180-\phi_{1}-\epsilon\right) \tan \left(180-\phi_{2}-\epsilon\right) \text { (for quadrants II } \\
\text { and III) } \\
4 \epsilon=720-\phi_{I}-\phi_{I I}-\phi_{I I}-\phi_{1 V}\end{array}$} & $\begin{array}{c}T=\cot \theta_{1} \\
\tan \theta_{2} \\
4 \theta=360 \\
+\phi_{1}-\phi_{11} \\
+\phi_{111}-\phi_{1 V}\end{array}$ \\
\hline 1 per cent sectored disk .- & $\left\{\begin{array}{c}m \mu \\
490 \\
530 \\
570 \\
610 \\
650\end{array}\right.$ & $\begin{array}{r}T_{1} \\
0.00855 \\
.00873 \\
.00873 \\
.00866 \\
.00867\end{array}$ & $\begin{array}{r}T_{\mathrm{II}} \\
0.00848 \\
.00848 \\
.00855 \\
.00854 \\
.00838\end{array}$ & $\begin{array}{r}T_{\mathrm{III}} \\
0.00845 \\
.00860 \\
.00869 \\
.00846 \\
.00863\end{array}$ & $\begin{array}{c}T_{\mathrm{zV}} \\
0.00840 \\
.00862 \\
.00884 \\
.00873 \\
.00849\end{array}$ & $\begin{array}{c}T_{\mathrm{Mean}} \\
0.00847 \\
.00861 \\
.00870 \\
.00860 \\
.00854\end{array}$ & $\begin{array}{r}0.00848 \\
.00860 \\
.00870 \\
.00860 \\
.00856\end{array}$ \\
\hline 10 per cent sectored disk. & $\left\{\begin{array}{l}530 \\
530 \\
610\end{array}\right.$ & $\begin{array}{l}.0994 \\
.1005 \\
.0984\end{array}$ & $\begin{array}{l}.0995 \\
.1004 \\
.1005\end{array}$ & $\begin{array}{l}.0993 \\
.0999 \\
.0990\end{array}$ & $\begin{array}{l}.0996 \\
.1000 \\
.1004\end{array}$ & $\begin{array}{r}.0994 \\
.1002 \\
.0996\end{array}$ & $\begin{array}{l}.0995 \\
.1002 \\
.0996\end{array}$ \\
\hline
\end{tabular}

(e) Procedure in Measurements.-The usual procedure in a transmission or reflection measurement at a given wave-length setting is as follows: After the wave-length scale has been set to the desired position, 5 settings of the angle of match $\theta_{1}$ are made in the 
first quadrant with the sample in (say) beam 1 . The sample is then shifted to beam 2 , and 10 settings of the angle $\theta_{2}$ are made. Returning the sample to beam 1 again, the group of 10 settings of $\theta_{1}$ is completed. As these readings are made they are immediately recorded by the observer on an (8-place) adding machine, the keyboard of which is split so that $\theta_{1}$ and $\theta_{2}$ may be recorded in separate columns. This machine is shown in Figure 1, conveniently situated for the observer's right hand. After the two sets of readings at a particular wave length are completed, a pull on the lever adds these readings, and thus records the mean angles. When a complete set of measurements have been made at various wave lengths throughout the spectrum, the values of $X$ are computed with a special slide rule having a cotangent and tangent scale. ${ }^{22}$

In the case of some reflection samples the intense illumination from the hemispherical source may, as previously noted, cause changes in the reflective properties of the sample during the course of the measurements. In order to detect these changes and to make an

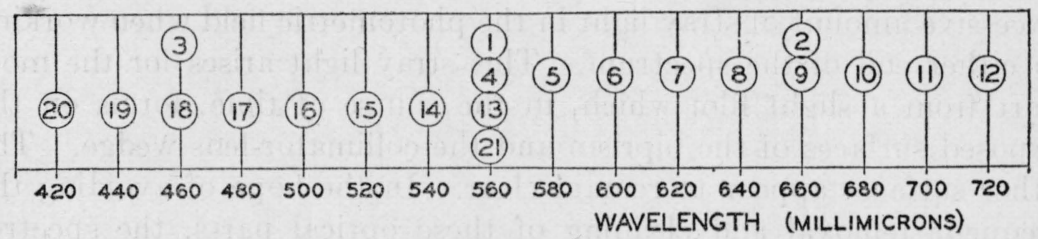

Fig. 16.-Order of determinations with respect to wave length, generally followed in reflection measurements

approximate correction therefor, a definite predetermined order of observation with respect to wave length is always followed. The procedure may vary with the type of sample to be measured and with other conditions; but the order of observation generally followed is depicted in Figure 16 and represents a minimum set of observations for a given reflection sample. If the determinations 1 and 4 (at wave length 560) show no change, for instance, but a definite difference is found between determinations 2 and 9 (at wave length 660), one may generally assume, to a first approximation at least, that the change has been progressive with time and that proportional changes have taken place at the intervening or adjacent wave lengths. Sometimes the reverse changes are noted. If there is reason to suspect that the sample is particularly subject to change in the blue region of the spectrum, the series 13 to 20 may precede 4 to 12 . A more elaborate arrangement of the observations may be devised in particular cases.

${ }_{22}$ Made to order by Keuffel \& Esser Co., Hoboken, N. J., after design by I. G. Priest at the Bureau of Standards. 
It is difficult to establish any fixed order of observation for all samples or rules for the correction of the data. The experience and judgment of the observer determines the exact procedure followed with a given kind of material, type of reflection curve, or other circumstances. Usually the changes, when they do occur, are not more than a few per cent of the value measured, and the approximate corrections derived from a study of the observed changes at a few

- chosen wave lengths are better than none whatever. The nature of the materials usually does not warrant more accurate data than are obtained in this way. In cases wherein a large change does occur it is often desirable to repeat some of the measurements with the use of a new and unexposed sample of the material.

\section{FURTHER REMARKS ON THE INSTRUMENT AND ITS ADJUSTMENTS}

(a) Stray Light.-Although the optical parts of the instrument are well inclosed by the manufacturer, it was formerly necessary to remove some of these parts during the course of each year in order to clean the optical surfaces and thus prevent the appearance of an excessive amount of stray light in the photometric field when working in either end of the spectrum. This stray light arises for the most part from a slight film which, in the course of time, forms on the exposed surfaces of the biprism and the collimator-lens wedge. The other surfaces appear to remain clear. In the hope of avoiding the frequent removal and cleaning of these optical parts, the spectrophotometer has been inclosed in a dust-proof and approximately moisture-proof box, which is shown in Figure 1. (Door shown open ready for measurements.) This housing prevents the accumulation of dust on the instrument and has proved very effective in retarding the development of stray light in the photometric field. Several glass light filters are provided, having transmission bands in different regions of the spectrum. These filters may be placed between the eye and the nicol and practically eliminate any small amount of stray light present.

(b) Photometric Field.--Under ordinary conditions of measurement and with the sources of light herein employed the photometric field of the instrument is well-nigh perfect, with a complete disappearance of the dividing line when both parts of the field are matched in brightness. The photometric precision of the brightness matches is believed to be as good as can be obtained with any other type of photometric field used in spectrophotometry.

There is one experimental condition, however, under which very imperfect field conditions will be obtained unless care is taken in the adjustment of the lens-Wollaston-biprism combination (hereafter referred to by the letters LWB). This condition often arises in transmission or reflection measurements on materials exhibiting a strong 
selective absorption, so that the spectral distribution of the light entering one of the collimator slits of the instrument is varying rapidly with the wave length in certain regions of the spectrum, whereas the variation with wave length of the light entering the other collimator slit is comparatively small. The extremely rapid variation in the spectral light distribution of the entrant beam may be accompanied by an appreciable variation in brightness across the corresponding half of the photometric field, this variation being always in a direction parallel to the dividing line (vertical). The brightness in the other half of the field will, on the other hand, appear sensibly uniform. This brightness variation may be directly attributed to two possible causes, both producing the same effect. These causes are: (1) The inaccurate focusing of the telescope lens, and (2) the spherical aberration present in the lens system of the instrument.

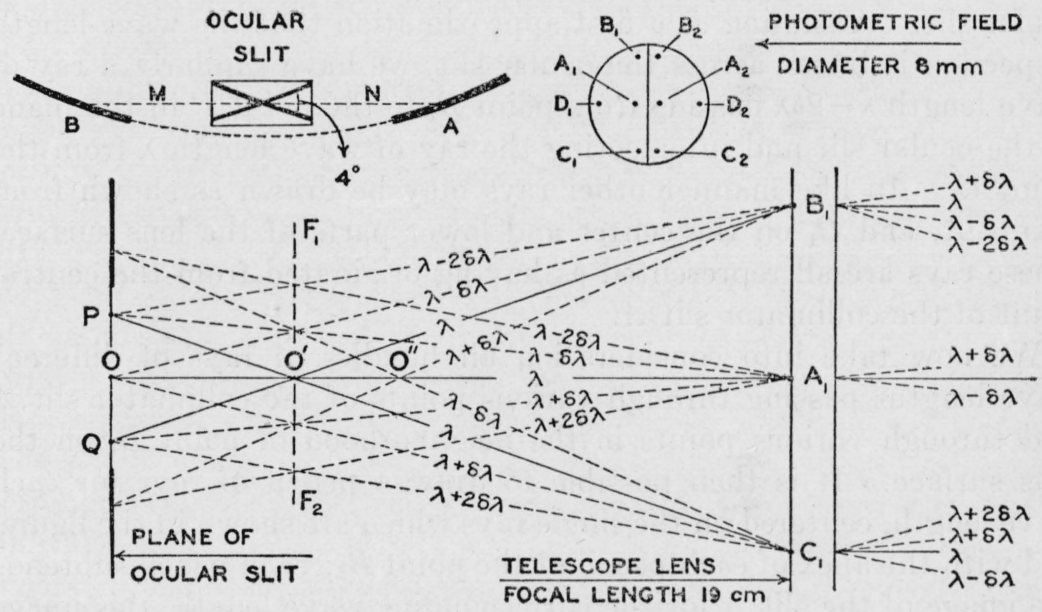

FIG. 17.-Conditions resulting in a nonuniform brightness distribution over the photometric field

An explanation of the effect will now be given, along with the proper procedure to be followed in the adjustment of the LWB combination in order that the above causes may have a minimum effect on the uniformity of the brightness distribution over the photometric field.

In the first part of this discussion all defects which may be present in the lens system of the instrument will be neglected. The LWB combination is regarded as a perfect lens. We recall, however, that, as viewed through the ocular slit, the lens surface is divided into two equal parts by the line of intersection of the biprism faces (dividing line in photometric field; see fig. 17, upper right) and each part is independently illuminated by light entering the corresponding collimator slit (fig. 3). Let the wave-length scale of the instrument be set for a particular wave length $\lambda$ and let the entrant beams be heterogeneous with respect to wave-length composition. 
Consider a homogeneous bundle of rays of wave length $\lambda$ entering the instrument through the central point of the collimator slit $A$. These rays may be assumed in the present discussion to be incident on the telescope lens, in Figure 17, as a parallel beam which is itself parallel to the axis of the lens; they are brought to a focus at the point $O^{\prime}$ on the axis and in the focal plane $F_{1} F_{2}$ of the lens. Let the ocular slit, which is centered on the axis of the lens, be displaced a distance $O O^{\prime}$ from its correct position in the focal plane. Consider, now, another homogeneous bundle of rays of wave length $\lambda-\delta \lambda$, originating also at the central point of the slit $A$. These rays will be slightly inclined to the axis of the telescope lêns and also brought to a focus at a point in the plane $F_{1} F_{2}$. The wave-length difference $\delta \lambda$ may be so chosen that the ray which passes through the point $B_{1}$ near the periphery of the lens will also pass through the central point $O$ of the ocular slit. Assuming as a first approximation that the wave-length dispersion is linear across the ocular slit, we have similarly a ray of wave length $\lambda-2 \delta \lambda$ passing from point $B_{1}$ to the point $P$ in the plane of the ocular slit and intersecting the ray of wave length $\lambda$ from the point $C_{1}$. In like manner other rays may be drawn as shown from points $A_{1}$ and $C_{1}$ on the center and lower parts of the lens surface. These rays are all represented as having originated from the central point of the collimator slit $A$.

We now take into consideration all bundles of rays of different wave lengths passing through various points of the collimator slit $A$ and through various points in the neighborhood of point $B_{1}$ on the lens surface. It is then possible to draw a pencil of rays for each wave length, centered on the single rays which are shown in the figure, and with the apex of each pencil at the point $B_{1}$; each pencil subtends the image of the slit $A$ for the corresponding wave length, this image being here considered as formed only by rays passing through the surface of the lens in the immediate neighborhood of the point $B_{1}$. The slit images for different wave lengths are displaced vertically relative to one another to form a sharply focused spectrum in the plane $F_{1} F_{2}$, but a slightly out-of-focus spectrum in the plane of the ocular slit. Considering all the rays passing through any other small area of the lens surface, as at points $A_{1}$ and $C_{1}$, a similar construction of rays may be made. It is evident from Figure 17 that the various spectra thus formed by various portions of the telescope lens coincide exactly in the plane $F_{1} F_{2}$, but are not coincident in the plane of the ocular slit. The spectrum formed in the latter plane by the upper part of the lens, at $B_{1}$, is shifted downward toward the longer wave lengths by an amount $\delta \lambda$, relative to the spectrum formed by the central part of the lens. Similarly, the spectrum formed by the lower part of the lens, at $C_{1}$, is shifted upward toward the shorter wave lengths by (approximately) the same amount. In addition to 
the vertical shift, there is also a lateral shift of each spectrum relative to the spectrum formed by the central part of the lens, this shift depending on the horizontal distance from the center of the lens of the image-forming portion under consideration. As will be shown later, the effect of the horizontal shift on the brightness variation across the photometric field is entirely negligible.

Looking through the ocular slit at a central portion of lens surface in the neighborhood of point $A_{1}$ (or $A_{2}$, fig. 17), one sees this area illuminated by light of mean wave length $\lambda$, but extending over a spectral range depending primarily on the widths of the collimator and ocular slits being used. The image on the retina of the eye is formed with all the homogeneous ray pencils, previously defined, originating at points in the area about $A_{1}$ and subtending the whole or a part of the ocular slit. The image of an upper portion of the lens surface in the neighborhood of point $B_{1}$ (or $B_{2}$ ) is formed with light of approximately the same range of wave lengths, but the mean wave length in this range is lower by $\delta \lambda$ than the mean wave length $\lambda$ at the center of the lens. Similarly, for a lower portion of the lens surface in the neighborhood of point $C_{1}$ (or $C_{2}$ ), the mean wave length is higher by $\delta \lambda$. There is no appreciable variation in mean wave length in a horizontal direction across the photometric field. The angle $Q B_{1} O$ (which is equal to $P C_{1} O$ ) is practically independent of $\lambda$; but the wave-length shift $\delta \lambda$, which corresponds to the linear distance $O P$ (or $O Q$ ) in the spectrum, increases rapidly with an increase in $\lambda$ as a result of the decrease in the linear dispersion in the spectrum toward the longer wave-length region. Hence, the shift in the mean wave length over the photometric field increases as the ocular slit is moved toward the longer wave lengths.

Now, let the spectral light distribution in beam 1 (say) be decreasing very rapidly with the wave length, whereas the variation with wave length of the light in beam 2 is, on the other hand, comparatively small. Then, if the nicol is set for equality of brightnesses at $A_{1}$ and $A_{2}$, where the mean wave length is $\lambda$, there will be an appreciably lower brightness in the upper part of field 1 at point $B_{1}$, where the mean wave length is $\lambda-\delta \lambda$, and a higher brightness at $C_{1}$, where the mean wave length is $\lambda+\delta \lambda$. Unless the ocular slit is very badly out of focus, the small wave-length shift $\delta \lambda$ will cause no appreciable difference between the brightnesses at points $A_{2}, B_{2}$, and $C_{2}$.

It is obvious from Figure 17 that the magnitude of $\delta \lambda$ may be increased or decreased by moving the ocular slit parallel to the axis of the lens, so that the distance $O O^{\prime}$ is increased or decreased. Thus, when $O O^{\prime}$ is zero, $\delta \lambda$ is also reduced to zero; hence, the photometric field must appear uniform in brightness. When the slit is moved in beyond the point $O^{\prime}$, the brightness gradient across the field is, of course, reversed, 
We will now consider the effect of any spherical aberration which may be present in the lens'system of the instrument. In order to use Figure 17 for this purpose, any effect of the collimator lens will not be represented. The spherical aberration of this lens would but increase the effect introduced by the telescope lens. Chromatic aberration and other defects in the optical system are of minor importance in this connection, and are therefore neglected in the following discussion.

Let a narrow pencil of rays of wave length $\lambda$, passing through the central part of the lens, be brought to a focus at $O^{\prime}$, as before. As a matter of convenience in the diagram, we may choose the distance $O^{\prime} O^{\prime \prime}$ to represent the longitudinal aberration of the lens. Then, a pencil of rays of wave length $\lambda$ from a peripheral part of the lens at $B_{1}$ will be brought to a focus at a point slightly above the axis of the lens on the line $B_{1} O^{\prime \prime}$. (The locus of all the foci of these narrow pencils of rays from various portions of the lens would form the caustic surface of the lens for the wave length $\lambda$.) Similarly, a ray $B_{1} O^{\prime}$ of wave length $\lambda-\delta \lambda$ may be drawn coincident with the ray of wave length $\lambda$ in the previous discussion. The ray $C_{1} O^{\prime}$ is now of wave length $\lambda+\delta \lambda$, and, likewise, other rays shown in the diagram are shifted in wave length by the same amount $\delta \lambda$. It is evident that the previous discussion applies identically to this case, so that the aberration effect is minimized by moving the center of the ocular slit to the point $O^{\prime \prime}$.

It is probable that the aberration effect is small, if not negligible, and that the focusing effect accounts for the greater part of the brightness gradient which may be observed in the photometric field under the conditions described above.

As illustrated in Figure 17 (upper left), the images of the two collimator slits do not coincide over their entire length. At their closest contact they make an angle of approximately $4^{\circ}$ with each other. It is of interest to consider what relation, if any, this crossing of the images may have on the focusing (or aberration) effect discussed above. This crossing of the images is a result of the curvature in the plane of the image, introduced by the action of the dispersion prism. If the biprism and Wollaston prism were removed from the instrument, the images of the two (separated) collimator slits would lie on the arc of a circle as shown at $A$ and $B$ in the figure. The Wollaston and biprism combination, however, in addition to dividing: these images into eight separate images, as previously explained, shifts them horizontally, causing two of them to overlap as shown at the center of the ocular slit. The horizontal shift of the spectra formed by the lateral portions of the telescope lens would obviously result only in a slight difference between the mean wave lengths at corresponding points, such as $D_{1}$ and $D_{2}$, in the lateral areas. The 
crossing of the images will also broaden the total wave-length range included by the ocular slit. In this respect it is equivalent to a widening of the ocular slit in the diagram. This does not change the distance $O O^{\prime}$ or therefore the value of $\delta \lambda$. The actual range of wave lengths above and below the average is increased, however, equally over all parts of the lens. If the rapidly varying spectral light distribution in the entrant beam is not a linear function of the wave length, it is possible that the brightness gradient across the lens may thereby be slightly increased or decreased. Experiment shows, however, that a change in the ocular-slit width, or a decrease (by use of a diaphragm) in the length of the slit, has very little effect on the appearance of the field.

The brightness gradient is never present when a homogeneous source, such as the mercury arc, is used for the illumination of the collimator slits. If the collimator-slit images were exactly parallel to the ocular-slit jaws, however, and if the edge of the ocular slit were then allowed to encroach slightly on the image of the spectral line, a brightness gradient across the lens would be observed. This is evident from a consideration of the $\lambda$ rays only in Figure 17, letting the distance $P Q$ represent the image of the collimator slit formed by the central portion of the telescope lens. The shifted image formed by a peripheral portion of the lens is obviously partly cut off by one edge of the slit, causing a decreased brightness in the corresponding part of the photometric field. (The duplication of this pattern for infinitesimal wave-length steps, as when a continuous-spectrum source is used, results in the somewhat different brightness gradient previously discussed.) It may be noted that the shift of the slit images is greatly exaggerated in the diagram. Unless the slit is very far from the plane $F_{1} F_{2}$, a rather nice adjustment of its edges would be necessary in order to observe any brightness gradient across the field, when a line-spectrum source is used. The crossing of the slit images, however, never permits the required relation to exist.

The brightness gradient can not be completely eliminated in the Koenig-Martens spectrophotometer, owing to the asymmetry introduced into the optical system by the wedges on the collimator and telescope lenses. In Figure 18 it is shown that the rays passing the upper parts of the telescope lens suffer excessive refraction and cross the axis of the lens between the point $O^{\prime}$ and the lens. Similarly, the rays passing the lower parts of the lens cross the axis slightly beyond the point $O^{\prime}$. The aberration, if present, is consequently also greater for the upper parts of the lens and less for the lower parts than that previously represented in Figure 17. There is no position of the ocular slit, therefore, for which the whole field is equal in brightness to the central portion. With the center of the 
slit at $O^{\prime}$ the brightness gradient from the central toward the lower portions of the photometric field is reversed, whereas the gradient toward the upper portions is not entirely eliminated. Hence, it is evident that both the lower and upper parts of the field will be somewhat darker than the central part.

This condition of the photometric field is not serious, and the importance of the residual brightness gradient, considered as an inherent defect in this particular type of instrument, must not be overrated or adjudged by the length of the discussion here given to it. The residual effect is only occasionally observed in transmission measurements under the condition previously stated. It is entirely absent when the mercury-vapor or helium lamp sources are used, as previously explained. In the case of reflection measurements the required condition of the entrant beam is rarely present. The effect introduces no error in the spectrophotometric measurements, if only

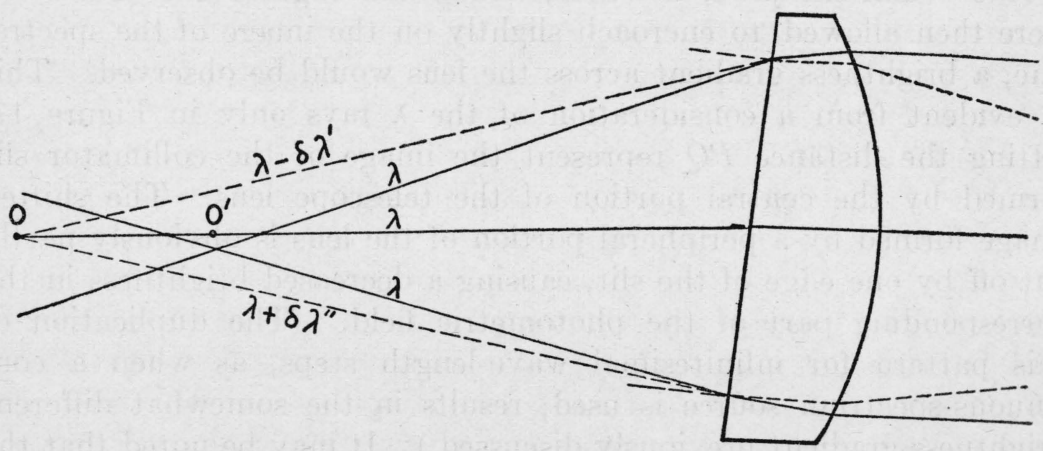

FIG. 18.-Effect of the asymmetry of the lens system on the brightness gradient across the photometric field

the central portion of the photometric field (which is free from the disturbing effects above described) is used for the brightness matches. But the precision of the settings of the angle of match is somewhat reduced, owing to the decreased area of the field and the decreased length of the dividing line which may be used.

The brightness gradient may well become a serious matter in the use of the Koenig-Martens spectrophotometer, however, if more than ordinary care is not taken in the adjustment of the distance of the $L W B$ combination from the ocular slit. The slit being stationary in this instrument, the $L W B$ combination must be moved along the axis of the telescope tube. The ordinary method of adjustment, using an eyepiece to view the ocular slit and adjusting the position of the lens so that the image of a spectral line is apparently as sharp as possible, is not sufficiently sensitive for the purpose. A better adjustment may be made in the following manner: 
The condition for the appearance of the brightness gradient is first established by inserting the proper type of (transmission) sample in one of the entrant beams. A high-transmission red glass with a sharp cut-off in the orange region of the spectrum is a suitable sample for this purpose. If the wave-length scale is set to a wave length on the steep slope of the transmission curve, the brightness gradient will generally be visible when the continuous-spectrum source of light is used. The $L W B$ combination is mounted in a brass tube which fits snugly into the telescope tube and is held in place by a clamping screw. Loosening this screw, the combination may be moved along the axis of the tube and also rotated about this axis. The combination is moved toward or away from the slit until the brightness gradient is reduced to a minimum. During this operation the center of the photometric field may be kept matched in brightness by rotation of the nicol; also, the angular position of the combination must be properly maintained such that no color difference appears between the two halves of the field. This color difference appears when the two line images of the collimator slits (fig. 17, upper left) are not crossing approximately at the center of the ocular slit, for it is evident that when this condition is not fulfilled the two spectra formed with all the light passing fields 1 and 2, respectively, are then shifted vertically and in opposite directions relative to each other. The mean wave length of the small spectral range transmitted by the ocular slit is then different for each spectrum, and consequently for each half of the photometric field. The image will cross at the center of the slit when the intersecting line of the biprism surfaces (dividing line in the photometric field) is perpendicular to the line $M N$.

The final adjustment of the angular position of the $L W B$ combination may be made more accurately by removing the red glass from the entrant beam and setting the wave-length scale in the neighborhood of wave length $580 \mathrm{~m} \mu$, for in this region of the spectrum a small change in wave length produces a maximum change in color. The final adjustment is best made, however, by illuminating the collimator slits with a line-spectrum source (such as the mercury-vapor lamp), then replacing the nicol with the eyepiece in order to view the slit images in the plane of the ocular slit, and finally making any rotation of the $L W B$ combination necessary to obtain a crossing of the images at the center of the slit. The complete adjustment of the $L W B$ combination is easily accomplished, and when once made is very stable.

(c) Wave-Length Scale.-Occasional attention to the adjustment of the wave-length scale is necessary. For this purpose the eyepiece is again used to view the-ocular slit. Using the homogeneous light from the helium and mercury-vapor lamps, the check or recali$109431^{\circ}-28-5$ 
bration of the scale is quickly made by finding the scale reading for which the crossed images of the collimator slits are centered on the ocular slit.

(d) Circular Scale.--The circular scale on the nicol mounting is large enough (75 $\mathrm{mm}$ in diameter) so that tenths of a degree may easily be estimated or read with the vernier. A low-power reading lens is provided for this purpose. This scale, with its single vernier, is sufficiently accurate for photometric purposes (when the regions close to 0 and $90^{\circ}$ are avoided, as previously discussed). Successive settings of the nicol can not generally be made closer than $0.2^{\circ}$; and in consequence of some obscure subjective vagaries of the human visual system the mean of a number of settings of the angle of match may vary as much from time to time.

(e) General Remarks. - The instrument is compactly made and is convenient to use. As shown in the vertical median section of Figure 3, the refracting edge of the dispersion prism is horizontal, giving the telescope tube an upward slant. The observer thus takes a convenient and natural position at the instrument, which adds considerably to his comfort during a long continuous series of observations, and hence to the quality of his work. The greatest disadvantage in this type of instrument is the waste of light inherent in the nature of the optical system. Some minor mechanical imperfections do exist, as in the construction of the ocular slit; because of the method employed for the mounting of the nicol prism this slit is somewhat inaccessible and is not provided with a means for quick and accurate adjustment in width. Whereas some improvements in the construction of its mechanical parts could be suggested it is doubtful if such changes would increase the general accuracy of measurement attainable with the instrument. Other matters in visual spectrophotometry would warrant a corresponding consideration.

\section{USE OF EQUIPMENT AND TESTS OF ITS PERFORMANCE}

\section{TRANSMISSION MEASUREMENTS}

The tungsten lamp (continuous spectrum) source is for obvious reasons used in the greater part of the transmission work. The maximum brightness of this source (88 lamberts) is more than sufficient for measurements in the more luminous region of the spectrum, so that the lamps are usually operated below normal voltage, except in the blue and extreme red ends of the spectrum or for low-transmission samples.

Many of the materials presented for transmission measurements (such as dye solutions, colored glasses, etc.) often exhibit rapid variations of transmission with wave length (steep transmission 
curves) in certain regions of the spectrum. In these cases a small spectral range (narrow collimator and ocular slits) must generally be used in order to avoid slit-width errors ${ }^{23}$ in the measurements. Except on the steep sides or near the minima or maxima of strong absorption bands, a spectral range of about $5 \mathrm{~m} \mu$ is generally safe for all spectrophotometric work. The slit widths on the KoenigMartens instrument corresponding to a 2 and $5 \mathrm{~m} \mu$ spectral range ${ }^{24}$ are given in Table 2. By experience the observer learns to adjust the slit widths to suit the conditions of measurement, thus obtaining in many instances the advantage of greater field brightness when needed without introducing appreciable errors in the work.

TABLE 2.-Widths of ocular slit on the Koenig-Martens spectrophotometer, for the inclusion of a given wave length range of pure spectrum

\begin{tabular}{|c|c|c|c|c|c|}
\hline \multirow{2}{*}{$\begin{array}{l}\text { Wave } \\
\text { length }\end{array}$} & \multicolumn{2}{|c|}{$\begin{array}{l}\text { Slit widths in mm } \\
\text { for spectral range } \\
\text { of- }\end{array}$} & \multirow{2}{*}{$\begin{array}{l}\text { Wave } \\
\text { length }\end{array}$} & \multicolumn{2}{|c|}{$\begin{array}{l}\text { Slit widths in mm } \\
\text { for spectral range } \\
\text { of- }\end{array}$} \\
\hline & $2 \mathrm{~m} \mu$ & $5 \mathrm{~m} \mu$ & & $2 \mathrm{~m} \mu$ & $5 \mathrm{~m} \mu$ \\
\hline $\begin{array}{r}\mathrm{m} \mu \\
720 \\
680 \\
640 \\
600 \\
560\end{array}$ & $\begin{array}{r}0.04 \\
.05 \\
.06 \\
.07 \\
.09\end{array}$ & $\begin{array}{r}0.10 \\
.12 \\
.14 \\
.18\end{array}$ & $\begin{array}{c}\mathrm{m}_{\mu} \\
520 \\
480 \\
440 \\
420 \\
400\end{array}$ & $\begin{array}{c}0.12 \\
.16 \\
.23 \\
.29 \\
.36\end{array}$ & $\begin{array}{r}0.30 \\
.40 \\
.58 \\
.71 \\
.91\end{array}$ \\
\hline
\end{tabular}

In this connection the measurements with the helium and mercuryvapor lamp sources are of special value as checks on the measurements made with the heterogeneous (tungsten lamp) source. With the homogeneous light from these sources the slits may be opened wide without danger of slit-width errors and with a proportional gain in field brightness. The distribution of the $\mathrm{Hg}$ and $\mathrm{He}$ wave lengths usually permits a check on the steep part of the transmission curves.

The availability of the $\mathrm{Hg}$ lines at wave lengths 436 and $405 \mathrm{~m} \mu$ is of further advantage in supplementing the continuous-spectrum source in the blue and violet regions of the spectrum, where the field brightness with the latter source is usually too low for best photometric work. Using these homogeneous radiations, however, precise measurements may be made at the corresponding wave lengths for transmissions as low as 1 per cent at wave length $405 \mathrm{~m} \mu$ and lower at $435 \mathrm{~m} \mu$. This matter is of considerable importance in the computa-

${ }^{23}$ See discussion of slit-width errors in papers by Nichols and Merritt, Phys. Rev., 30, p. 328; 1920. Hyde, E. P., Astrop. J., 35, p. 237; 1912.

24 The spectral range included by the ocular slit is somewhat indefinite until further conditions are specified. The data in Table 2 are computed from the wave-length calibration curve of the instrument and represent the ocular-slit widths which would include a given range of a pure spectrum such as would be formed if the collimator slit were very narrow. When the collimator-slit width is equal to that of the ocular slit, the actual range of wave lengths in the transmitted light is doubled, owing to the overlapping of collimator-slit images at the ocular slit; but a greater part of the total radiant energy transmitted by the ocular slit is distributed among the wave lengths contained in the pure spectrum range given in Table 2. 
tion from the spectrophotometric data of the trichromatic coordinates of the color of the transmitted light, as well as in other applications of the spectrophotometric data. The change to the helium or mercury-vapor sources is rapidly and conveniently made, as previously explained.

As an indication of the precision and accuracy of the measurements with the spectrophotometer and transmission sources herein described, the measured transmissions of the 1, 10, 50, and 80 per cent sectored disks are shown in Figure 19 along with the fractional probable errors in the transmission as computed by equation (42). The precision of measurement depends to some extent, of course, on the general brightness level of the photometric field. In order, therefore, that the field brightnesses in these tests be representative of general working conditions with the heterogeneous source, a spectral range of $5 \mathrm{~m} \mu$ was maintained at all wave lengths with the following exceptions: At wave lengths 700,720,740, and $760 \mathrm{~m} \mu$ the ranges were $7,10,16$, and $18 \mathrm{~m} \mu$, respectively. With the mercury-vapor lamp source, slits as wide as possible were used for wave lengths 436 and 405 $\mathrm{m} \mu$. In this way a greater field brightness is obtained at these wave lengths than can be obtained with the heterogeneous source and narrow slits. For wave lengths 546 and $578 \mathrm{~m} \mu(576.9+579.1 \mathrm{~m} \mu)$ the field brightness with the wide slits was too great for eye comfort. In these cases the slits were either narrowed or a neutral low-transmission screen placed before the nicol. The precision of the measurements made with the $\mathrm{Hg}$ lines at wave lengths 436 and $405 \mathrm{~m} \mu$ is, in general, as good, even for low-transmission samples, as that obtained with the heterogeneous source in the more luminous regions of the spectrum.

The horizontal broken lines in Figure 19 represent the known transmissions of the disks as given by angular measurements of the sector openings. ${ }^{25}$ These values are given in the figure and are known to be accurate within one unit in the last decimal place. The accuracy of the measurements with the spectrophotometer is indicated by the general proximity of the points to horizontal lines. Occasional wide departures, which do not appear to be caused by inherent instrumental errors, are common in spectrophotometric work and are often difficult to explain. Measurements on these disks, made by other observers in this laboratory on various occasions, are in good agreement with the above data. The data, as a whole, show no systematic error in the measurements at any wave length and for transmission values down to 10 per cent. Measurements on a sectored disk having a known transmission of 0.0154 have been reported in a previous publication. ${ }^{26}$ These measurements indicate no discrepancies in the direct measurement of low-transmission values of this magnitude. 
In the case of the 1 per cent sectored disk (accurate transmission $=$ 0.88 per cent), however, the measurements herein reported are consistently too low by an average value of about 2 per cent. Referring to the data in Table 1, it is evident that this error is not caused by the inaccuracies there considered in the circular scale of the nicol mounting, for it is not eliminated by the averaging of the readings made in the four quadrants. In the previous discussion of errors in the circular scale it was assumed that the plane-polarized vibrations entering the nicol from fields 1 and 2 were strictly at right angles to each other; also, that the transmission of the nicol prism for a planepolarized beam followed exactly the cosine-square law. Neither of these conditions may be accurately fulfilled, and the discrepancies,

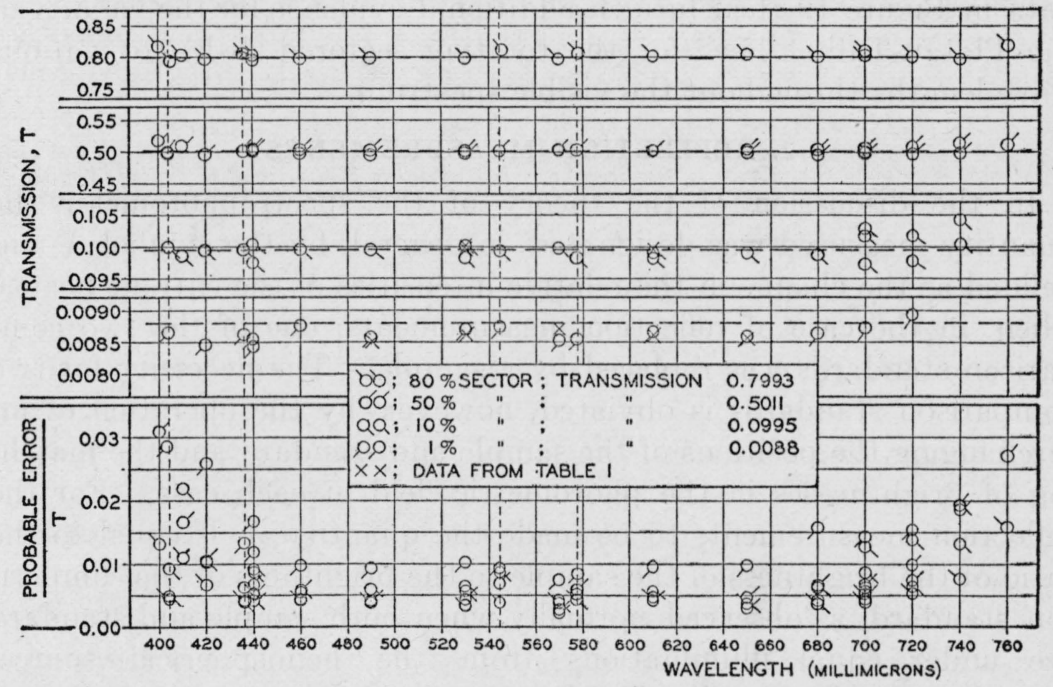

FIG. 19.-Test of precision and accuracy of measurements with the KoenigMartens spectrophotometer (using transmission sources)

though small, may readily give rise to the error observed in the direct measurement of such low-transmission values.

These errors are avoided, however, by use of the 1 or 10 per cent sectored disk in the beam opposite the sample, as previously described. In this way transmissions in the neighborhood of 0.001 are measured with accuracy, for all wave lengths at which a sufficient field brightness may be obtained.

To test the accuracy of the mechanical calibration of the 1 per cent sectored disk, its transmission was measured with the spectrophotometer relative to that of the 10 per cent disk. The following substitution method was used in order to avoid readings on the circular scale near 0 and $90^{\circ}$ : A glass having a transmission of approximately 10 per cent was mounted in the regular manner in one beam, with the 1 per cent sectored disk mounted in the other beam. 
The transmission of the disk was then carefully measured. (for wave length $530 \mathrm{~m} \mu$ ) relative to that of the glass by the usual method of interchanging the glass and sector in the beams. Ten readings of each angle of match were made in each quadrant of the circular scale. The 10 per cent disk was then substituted for the 1 per cent disk and. likewise measured relative to the same glass (without disturbing the position of the glass on the sample holder). From these data the transmission of the 1 per cent disk was computed, using the known transmission of the 10 per cent disk, as given by both mechanical and. photometric calibrations (fig. 19). A value of 0.00880 was obtained, which is in excellent agreement with the mechanical calibration.

Assuming the accuracy of the photometric measurements, the data in Figure 19 then furnish additional evidence for the validity of the Platau-Talbot law (for the rotating sectored disk) for various wave lengths throughout the visible spectrum.

\section{REFLECTION MEASUREMENTS}

In the discussion of the theory of the spectrophotometer the quantity measured was designated, in general, by the symbol $X$ and. defined as the change in the relative intensities of the entrant beams, when, in the case of reflection measurements, one of the two comparison standards was replaced by a sample. The necessity for two comparison standards is obviated, however, by the operation of interchanging the positions of the sample and standard and the matching of brightnesses in the photometric field in each case. For the reflection measurements to be made the quantity $X$ is equal to the ratio of the brightness of the sample to the brightness of the comparison standard, as observed normally when both sample and standard are under equal illuminations from the hemispherical source. Actually, the absolute illuminations on the sample and standard need. not be the same, such inequalities being effectually eliminated in either of the two methods of use of the instrument. (See Section IV , 3.) The brightness ratio may depend, however, on the degree of diffusion of this illumination. This matter will be fully discussed. läter in this section.

(a) Reflection Standards.-The comparison (or "working") standard which has been in use for some time is an opaque porcelain plate having a fine-ground (matt) reflecting surface. The choice of this particular material was purely a matter of convenience and serviceability. Its absolute reflectance is fairly high and approximately nonselective with respect to wave length; it is durable, withstands the heat from the lamps without special cooling, and may be removed for cleaning as frequently as desired.

In order to express the reflective properties of a given material in terms of a more definite and reproducible material, however, a magnesium oxide film is used as the primary reference standard for 
all spectral reflection work. This material is used pending the development of a more suitable material or means for the absolute evaluation of the working standard.

The magnesium oxide standard is prepared by allowing the oxide from burning metallic magnesium to deposit upon a plane matt porcelain plate to a thickness such that a further increment in thickness would produce no change in the reflective properties. A magnesium oxide film so prepared is a good diffuser and has a reflectance almost unity (approximately 0.98), with very little, if any, selectivity with respect to wave length.

No extensive study of the permanence or reproducibility of this standard has been,made. In order to calibrate the working standard, however, measurements of its brightness relative to that of the oxide have been made (with the equipment herein described), using five different oxide films. These films were prepared in the prescribed manner from two different commercial sources of magnesium metal shavings (as used for the Grignard reaction). The films varied in thickness from 0.36 to $0.50 \mathrm{~mm}$, and measurements were made in each case on the same day the films were prepared. Three different observers participated in these measurements. Three of the films were prepared and measured (by H. J. M.) soon after the equipment was completely installed. The other two films were prepared and measured two years later (by K. S. G. and D. B. J.). No consistent differences between these various determinations were found which could be definitely ascribed to real differences between the reflective properties of the various films. From the data obtained thus far, this standard appears to be reproducible, under the prescribed conditions of preparation and use, within the limits of accuracy of the spectrophotometric measurements.

Under the specified conditions of illumination and observation, then, let

$$
\begin{aligned}
& B_{0} \equiv \text { brightness of primary MgO standard, } \\
& B_{0}{ }^{\prime} \equiv \text { brightness of working standard, } \\
& B_{\mathrm{x}} \equiv \text { brightness of test sample. }
\end{aligned}
$$

In routine reflection measurements the quantity $B_{\mathrm{x}} / B_{0}{ }^{\prime}$ is directly measured. $B_{\mathrm{o}}{ }^{\prime} / B_{\mathrm{o}}$ is the result of the calibration of the working standard in terms of the primary standard. The final expression of the reflective properties of the sample is then computed as

$$
\frac{B_{\mathrm{x}}}{B_{0}}=\frac{\text { Brightness of sample }}{\text { Brightness of } \mathrm{MgO} \text { standard }}=\frac{B_{\mathrm{x}}}{B_{0}{ }^{\prime}} \frac{B_{\mathrm{o}}{ }^{\prime}}{B_{\mathrm{o}}}
$$

This ratio is, of course, a function of the wave length.

Referring to the discussion of Absolute Methods in Reflectometry in Bureau of Standards Journal of Research, vol. 1, No. 3, it is of interest to note in this connection that the brightness ratio measured 
in the present paper may also be expressed (in the terminology of the paper cited) as the apparent normal reflectance of the sample relative to that of the standard under the given conditions of completely diffused illumination. Also, in accordance with the reciprocity law of Helmholtz, the quantity measured is the reflectance of the sample relative to that of the standard for unidirectional normal illumination. If the absolute spectral reflective properties of the standard were known, the absolute spectral reflectances of the sample would then be determined. Equipment is now being assembled with which the standard may be calibrated in absolute reflective terms, if desired.

(b) Adequacy of Absolute Illumination on Sample.-The nature of the materials usually presented for reflection measurements is such that the above brightness ratio does not generally exhibit such rapid variations with wave length as does the transmission of many glasses, solutions, etc. In other words, reflection "curves" are not generally so steep as transmission curves. Without danger of appreciable slit-width errors, then, wider slits may generally be used in reflection measurements, with a corresponding gain in photometric field brightness. Another characteristic of reflection samples is that a measurable quantity of light is always reflected into the spectrophotometer at all wave lengths. Under completely diffused illumination this quantity is rarely less than 2 or 3 per cent of that reflected by the comparison standard. Hence the photometric field brightness at a given wave length is never reduced below a certain limit.

These characteristics of the reflection samples have a bearing on the adequacy of the hemispherical source herein described, in regard to the absolute illumination on the sample. Although, for a given value of $X$ and given widths of the instrument slits, the spectrophotometric field brightness with this source is not as high as that obtainable with the transmission sources, the illumination on the sample and standard is sufficient, nevertheless, for good photometric precision in the measurements throughout the brighter region of the spectrum. When proper advantage of the permissible slit widths is taken, measurements of sufficient precision may always be made over the whole spectral range from 700 or 720 to $420 \mathrm{~m} \mu{ }^{27}$

\footnotetext{
${ }^{27}$ An arrangement providing completely diffused illumination from a mercury-vapor lamp is desirable to supplement the above heterogenerous illumination, so that accurate measurements may be made on any sample at wave lengths 405 and $436 \mathrm{~m} \mu$ (as obtained in the case of transmission measurements). Apparatus has been installed in the laboratory, however, with which the desired brightness ratio may be measured under approximately $45^{\circ}$ illumination from the mercury-vapor lamp. The Martens photometer is used in this case with selective filters to isolate the various spectral lines. Proper precautions are also taken to avoid polarization errors in the measurements. When the diffusive properties of the sample are not widely different from those of the comparison standard, the normal brightness ratios obtained under these conditions of illumination are practically the same as obtained under completely diffused illumination. With this equipment the field brightness at wave length $405 \mathrm{~m} \mu$ is wholly adequate for precise reflection measurements on all samples.

This arrangement of apparatus was designed and assembled by Dr. K. S. Gibson especially for use in measurements made for the Munsell Research Laboratory on 70 representative samples from the Munsell Color System (B. S. test 46045; 1926). The method has subsequently been further developed and applied by W. D. Appel. See American Dyestuff Reporter (Sample Swatch Quarterly), p. 49; Jan. 23, 1928.
} 
'The hemisphere lamps are usually operated at a voltage considerably below normal, except in the end regions of the spectrum or for low-reflection samples.

(c) Equivalence of the Illumination to the Ideal Completęly Diffused Condition.- The measurements described on the sectored disks are a proof of the accuracy of the spectrophotometer in the measurement of the normal brightness ratio of the sample to the standard under the actual conditions of illumination from the hemispherical source. The question now arises whether or not the numerical value thus obtained truly represents the desired brightness ratio under the ideal condition of completely diffused illumination. This ideal condition would be realized if all elements of the hemispherical inclosure were of equal brightness. Obviously, the condition is not rigorously satisfied in the actual construction of the hemispherical source, but the tests to be described will show that the illumination obtained with the present construction is practically equivalent to the desired condition of illumination for the reflection measurements to be made on different kinds of materials.

If the sample and comparison standard have very nearly the same diffusive properties ${ }^{28}$ (but not necessarily the same reflectance), the normal brightness ratio would be the same for any condition of illumination. When the diffusive properties of the sample differ widely from those of the standard, however, the brightness ratio may depend to a considerable extent on the condition of illumination.

Assuming that the illumination is not a sufficient approximation to the desired condition, then the error in the measurement of the normal brightness ratio of the sample to the standard depends essentially on the following considerations: (1) The degree and nature of the departure of the illumination on the sample from the desired completely diffused condition, and (2) on the diffusive properties of the sample and comparison standard.

The comparison standard is usually a fairly matt diffusely reflecting material. The samples, on the other hand, may represent various stages of mixed diffuse and specular reflection varying from nearly perfect diffusion on the one extreme to pure specular reflection (mirror sample) on the other.

In the test of the illumination apparatus and in the choice of samples for this purpose we are not particularly concerned with the absolute reflectance of the test samples (or standard), or with the spectral composition of the incident and reflected light. These matters play no essential rôle in the test and enter into consideration

${ }^{28}$ Two samples will be considered to have the same diffusive properties under given conditions of illumination when the relative angular distributions of the reflected light flux-compared always at the same wave length - are practically the same for each sample. The diffusive properties depend to a large extent on the absolute reflectance of the sample; hence, when the latter property varies markedly through the spectrum the diffusive properties change accordingly. 
only implicitly through their relation to the diffusive properties of the test samples. For example, a sample of black paint with a smooth, glossy surface will approximate closely to the mirror sample, because very little light is reflected diffusely from the body of the material. Given a similar material, however, with a white pigment instead of the black, the large quantity of body reflection accompanying the surface reflection will bring the sample closer in type to the perfect diffuser. Likewise, the diffusive properties of a colored sample may be quite different for different parts of the spectrum.

A practical test of the adequacy of the hemispherical arrangement herein described, as a source of completely diffused illumination for the prescribed purposes, has been made in the following manner:

1. A separate source of diffused illumination was available, which was known by independent tests to be a very close and sufficient approximation to the completely diffused condition of illumination for these purposes.

2. A group of samples was chosen representing various degrees of departure from the perfectly diffusing sample on the one hand and a mirror sample on the other. The color of these samples being unimportant, a group of neutral samples was used of which the reflective properties were already known from the results of a previous investigation.

3. The brightness ratios of the test samples to the (porcelain) comparison standard were measured for both sources of diffused illumination and the results compared. An agreement between the two sets of measurements is then considered as a sufficient proof of the equivalence of the two illuminations for the intended purpose.

The known source for completely diffused illumination used in this test is part of an equipment now being assembled for the study of various problems involving the reflective and transmissive properties of diffusing materials. A complete description of the source and the spectrophotometric apparatus used with it will be given in a forthcoming publication of the bureau series. In the following brief description of this new equipment only those features are mentioned which have a bearing on its application to the present problem:

1. The source for completely diffused illumination is constructed in a manner somewhat similar to that described in the present paper, the essential difference respecting the illumination on the sample being the interposition of a milk-glass hemisphere between the lamps and the sample.

2. The sample and standard are not mounted side by side and compared simultaneously, but either one may be placed alone at the center of the base of the milk-glass hemisphere and the normal brightness of the sample observed with a spectrophotometer through 
a small hole at the pole of the hemisphere. The sample may also be observed at $12^{\circ}$ from the normal (and at five other angles).

3 . The spectrophotometer ${ }^{29}$ differs in type from the KoenigMartens instrument and uses a comparison source, separate from the hemispherical source, for the illumination of one half of the photometric field. The other half of the field is illuminated by light reflected from either the sample or the standard under the (completely) diffused illumination from the milk-glass hemisphere. The desired brightness ratio is obtained by alternate substitutions of the sample and standard.

4. The relative brightness distribution over the inner concave surface of the milk-glass hemisphere may be measured directly from the position of the sample and is known to be constant within definite limits, so that the degree of approximation to the ideal completely diffused condition of illumination on the sample is also definitely known.

This illumination is as nearly completely diffused as any of three diffused illuminations previously described by the author in connection with a study of some absolute methods in reflectometry. (B. S. J. Research, $\mathbb{1}$, No. 3, fig. 4.) In that work a group of samples. was used, approximately neutral in color, and representing various degrees of departure from the perfectly diffusing sample. The reflective properties of these samples were found to be the same under each of the three diffused illuminations above referred to. Hence it was concluded that the three illuminations were each a sufficient approximation to the completely diffused condition for all practical purposes. The reflective properties of the samples were not analyzed with respect to wave length, however, as a spectrophotometer was not used in the investigation. Hence the measurements therein made can not be compared directly with measurements which might be made on the same samples with the Koenig-Martens spectrophotometer and the illumination apparatus here under test. Different quantities are measured in each case. This previous work is referred to here as a proof of the equivalence of the illumination from the milk-glass hemispherical source to the ideal completely diffused condition of illumination, in order that the equipment briefly described above may be justly applied to the present test of the source of diffused illumination used with the Koenig-Martens spectrophotometer. Furthermore, the samples therein employed form a series such as is required in this test. The samples actually used, however, are not the identical samples employed in the previous work, but very similar pieces from the same stock or kind of material;

${ }_{20}$ The type of instrument used is described by C. W. Keuffel, J. Opt. Soc. and Rev. Sci. Inst., 2, p. $403 ; 1925$. 
so that the data and curves in the paper cited serve as a good description of the reflective properties exhibited by the test samples. These samples are all of a type showing no appreciable polarization of the light reflected to the spectrophotometer.

The brightness ratios, as measured with both sources of diffused illumination, are given in Table 3. From the preceding discussion it is evident that complete measurements for various wave lengths throughout the whole visible spectrum are not essential to this test. Measurements have been made only at wave lengths 480,580 , and $670 \mathrm{~m} \mu$. Slit widths on each spectrophotometer corresponding to a $10 \mathrm{~m} \mu$ spectral range were used to insure an entirely adequate field brightness and thus a maximum photometric precision in all the measurements. The use of neutral samples avoids the possibility of any slit-width errors or other uncertainties incident to the measurement of brightness ratios varying rapidly with the wave length. The magnesium-carbonate, white-felt, and $\mathbf{M}$ samples have a matt surface, whereas the $V$ samples are photographic papers having a smooth, very glossy surface.

TABLE 3.-Test of the diffused illumination used for reflection measurements, showing its equivalence to the ideal completely diffused condition of illumination

\begin{tabular}{|c|c|c|c|c|c|c|c|c|c|}
\hline \multirow{4}{*}{ Sample 1} & \multirow{4}{*}{$\begin{array}{l}\text { Wave } \\
\text { length }\end{array}$} & \multicolumn{8}{|c|}{$\frac{B_{\mathbf{x}}}{B_{\mathrm{o}}}=\frac{\text { Brightness of sample }}{\begin{array}{c}\text { Brightness of (porcelain) comparison standard } \\
\text { measured under the illuminations from- }\end{array}}$} \\
\hline & & \multicolumn{4}{|c|}{$\begin{array}{l}\text { Source used with Koenig- } \\
\text { Martens spectrophotometer } \\
\text { (shown in figs. } 3 \text { and } 4 \text { ) }\end{array}$} & \multicolumn{4}{|c|}{$\begin{array}{l}\text { Milk-glass hemispherical source } \\
\text { for completely diffused illu- } \\
\text { mination (briefly described in } \\
\text { text) }\end{array}$} \\
\hline & & \multicolumn{3}{|c|}{ Determinations- } & \multirow{2}{*}{ Mean } & \multicolumn{3}{|c|}{ Determinations- } & \multirow{2}{*}{ Mean } \\
\hline & & 1 & 2 & 3 & & 1 & 2 & 3 & \\
\hline \multirow{3}{*}{ Magnesium carbonate block } & & 1. 232 & 1. 231 & & 1.232 & 1. 255 & 1. 214 & & 1. 235 \\
\hline & 580 & 1. 230 & 1. 250 & & 1.240 & 1.260 & 1. 232 & & 1. 246 \\
\hline & 670 & 1. 294 & $\begin{array}{r}1.304 \\
758\end{array}$ & 0.752 & 1. 299 & 1.307 & 1. 300 & & 1. 303 \\
\hline \multirow{2}{*}{ White felt } & $\begin{array}{l}480 \\
580\end{array}$ & $\begin{array}{l}.756 \\
.846\end{array}$ & $\begin{array}{l}.758 \\
.850\end{array}$ & $\begin{array}{r}0.752 \\
.832\end{array}$ & $\begin{array}{l}.755 \\
.843\end{array}$ & $\begin{array}{l}.773 \\
.853\end{array}$ & $\begin{array}{l}.770 \\
.858\end{array}$ & $\begin{array}{r}0.760 \\
.854\end{array}$ & .768 \\
\hline & 670 & .918 & .936 & .932 & .92 & .946 & .93 & & 934 \\
\hline \multirow{3}{*}{ Black felt. } & $\begin{array}{l}480 \\
580\end{array}$ & 026 & .0305 & .0276 & $\begin{array}{l}.0290 \\
0260\end{array}$ & 0259 & $\begin{array}{l}.0283 \\
0256\end{array}$ & 0 & .0290 \\
\hline & $\begin{array}{l}580 \\
670\end{array}$ & .0261 & $\begin{array}{l}.0260 \\
.0327\end{array}$ & .0327 & .0327 & & .0346 & .0355 & .0350 \\
\hline & 480 & .618 & .638 & & .628 & .626 & .636 & & .631 \\
\hline \multirow[t]{2}{*}{ M-2 (matt paper) .. } & 580 & .630 & .630 & .636 & .632 & .628 & .638 & $\ldots$ & .633 \\
\hline & $\begin{array}{l}670 \\
480\end{array}$ & $\begin{array}{l}.626 \\
.0598\end{array}$ & $\begin{array}{l}.634 \\
.0584\end{array}$ & $-1-1-1$ & $\begin{array}{l}.630 \\
.0591\end{array}$ & $\begin{array}{l}.614 \\
.0518\end{array}$ & $\begin{array}{l}.645 \\
.0581\end{array}$ & .0615 & .630 \\
\hline \multirow[t]{3}{*}{ M-8 (matt paper)... } & 58 & .0526 & .0532 & $\cdots$ & .0529 & .0502 & .0497 & .0504 & .0501 \\
\hline & 670 & .0577 & .0578 & & .0578 & .0578 & .0564 & .0589 & .0577 \\
\hline & 480 & .856 & .855 & .858 & .856 & .876 & .857 & .846 & .860 \\
\hline V-I-A (glossy pal & 580 & .878 & .867 & .868 & .871 & .876 & .880 & .874 & .877 \\
\hline & $\begin{array}{l}670 \\
480\end{array}$ & $\begin{array}{l}.944 \\
2030\end{array}$ & $\begin{array}{l}.924 \\
.2080\end{array}$ & $\begin{array}{l}.940 \\
.2030\end{array}$ & $\begin{array}{l}.936 \\
.2047\end{array}$ & $\begin{array}{l}.940 \\
.2020\end{array}$ & $\begin{array}{l}.943 \\
.2005\end{array}$ & .2036 & .2020 \\
\hline$v$ & 580 & .2008 & .2065 & .2083 & $\begin{array}{l}.2052 \\
.2052\end{array}$ & .2090 & .1985 & .1985 & .2020 \\
\hline & 670 & 211 & .2170 & .2078 & .2121 & .20 & & .2048 & .20 \\
\hline & 480 & 0745 & .0718 & & .0732 & .0788 & .0845 & & .0816 \\
\hline & $\begin{array}{l}580 \\
670\end{array}$ & .0782 & $\begin{array}{l}.0798 \\
0852\end{array}$ & $-\cdots$ & .0790 & .0785 & .0784 & .0792 & $\begin{array}{r}.0787 \\
0890\end{array}$ \\
\hline Silvered-glass mirr & 580 & 1.096 & 1. 085 & $\cdots$ & 1.090 & 1.082 & 1.084 & & 1. 083 \\
\hline
\end{tabular}

1 See B. S. J. Research, 1, No. 3, for complete description of the samples and their reflective properties. The $\mathbf{M}$ samples are gray painted cardboard (matt surface). The $\mathbf{V}$ samples are glossy photographic papers. 
The measurements on the mirror and glossy samples, in particular, show quite conclusively that the desired condition of illumination is approximated so closely, with the hemispherical illumination apparatus here under test, that further approximation to the ideal condition would not appreciably alter the observed brightness ratios.

When the glossy samples are observed normally with the milkglass hemispherical source, there is a noticeable effect on the observed brightnesses caused by the (black) observation hole at the pole of the hemisphere. The effect is not appreciable with the hemispherical construction used with the Koenig-Martens spectrophotometer, because of the greater spread of the beams and the narrowness of the hemisphere slits (15, fig. 4). Consequently, the measurements with the milk-glass hemispherical source were made on all the samples at an observation angle of $12^{\circ}$ from the normal. However, the previous study of these test samples shows that, with the exception of the white-felt sample only, the brightnesses under approximately completely diffused illumination are sensibly the same for 0 and $12^{\circ}$ angles of observation. The slightly higher value obtained for the white-felt sample with the milk-glass hemispherical source is in accordance with the reflective properties of this material.

In the measurement of very glossy samples it is evident that a greater part of the light reflected into the spectrophotometer originates from the polar region of the hemisphere. Consequently, it is particularly important that the average brightness of the source in this region be approximately equal to the average brightness of the whole hemisphere. The filtering of the air which is drawn through the hemisphere for ventilation purposes is an important matter in the maintenance of the desired condition of illumination.

A set of reflection samples which have been calibrated under a known source of completely diffused illumination are a desirable adjunct to the equipment, in order to test and maintain an occasional check on the conditions of illumination.

Many features of the equipment herein described represent the accumulated experience of the colorimetry laboratory of the Bureau of Standards in regard to the requirements for visual spectrophotometry. In this respect the author gratefully acknowledges miscellaneous contributions and suggestions by I. G. Priest and K. S. Gibson which are embodied in the design of the apparatus.

Washington, January 22, 1928. 
